\title{
The chemistry of bisallenes
}

Henning Hopf* and Georgios Markopoulos

\author{
Review \\ Address: \\ Institute of Organic Chemistry, Technical University of Braunschweig, \\ Hagenring 30, D-38106 Braunschweig, Germany, fax: \\ $+49-(0) 531-391-5388$ \\ Email: \\ Henning Hopf ${ }^{\star}$ - h.hopf@tu-bs.de \\ ${ }^{*}$ Corresponding author \\ Keywords: \\ alicyclic; bisallenes; cyclic; cycloadditions; cycloisomerization; \\ isomerization; molecular complexity; step economy
}

Beilstein J. Org. Chem. 2012, 8, 1936-1998.

doi:10.3762/bjoc.8.225

Received: 01 August 2012

Accepted: 13 October 2012

Published: 15 November 2012

Dedicated to Professor Roald Hoffmann on the occasion of his $75^{\text {th }}$ birthday.

Associate Editor: J. A. Murphy

(C) 2012 Hopf and Markopoulos; licensee Beilstein-Institut. License and terms: see end of document.

\section{Abstract}

This review describes the preparation, structural properties and the use of bisallenes in organic synthesis for the first time. All classes of compounds containing at least two allene moieties are considered, starting from simple conjugated bisallenes and ending with allenes in which the two cumulenic units are connected by complex polycyclic ring systems, heteroatoms and/or heteroatom-containing tethers. Preparatively the bisallenes are especially useful in isomerization and cycloaddition reactions of all kinds leading to the respective target molecules with high atom economy and often in high yield. Bisallenes are hence substrates for generating molecular complexity in a small number of steps (high step economy).

\section{Table of Contents}

\section{Introduction}

Review

1. Acyclic conjugated bisallenes

1.1 Synthesis of hydrocarbons

1.2 Synthesis of functionalized systems

1.3 Spectroscopic and structural properties of conjugated bisallenes

1.4 The chemical behavior of conjugated bisallenes

1.4.1 Pericyclic reactions

1.4.2 Ionic reactions

1.4.3 Metal-induced reactions
2. Acyclic nonconjugated bisallenes

2.1 1,2,5,6-Heptatetraene and its derivatives

2.2 1,2,6,7-Octatetraene and its derivatives

2.3 Higher acyclic $\alpha, \omega$-bisallenes

2.4 The chemical behavior of nonconjugated, acyclic $\alpha, \omega$-bisallenes

3. Bisallenes with unsaturated spacer elements

4. Bisallenes containing ring systems

4.1 Aromatic bisallenes

4.2 Bisallenes connected by cyclic spacer groups

4.2.1 Semicyclic bisallenes

4.2.2 Endocyclic bisallenes

4.2.3 Exocyclic bisallenes 
5. Bisallenes as reactive intermediates

6. Bisallenes with heteroatoms in their tethers (heteroorganic bisallenes)

6.1 Thermally induced reactions of heteroorganic bisallenes

6.2 Transition metal-induced reactions of heteroorganic bisallenes

Conclusion

Acknowledgements

References

\section{Introduction}

As every student of organic chemistry knows, the early years of this branch of science were exciting and confusing at the same time. This is particularly true for the chemistry of aromatic compounds before Kekule finally established the hexagonal structure for benzene [1]. Benzene was discovered by Faraday in 1825 , and Kekulé published his concept, which would revolutionize organic chemistry, in 1865 (and his dreams about the benzene ring in 1890) [2]. In fact, the early structure proposals for benzene varied considerably and Loschmidt, a contemporary of Kekulé who was also interested in developing a structural theory for organic compounds, wrote his first benzene structure (1) in 1861 as shown in Figure 1 [3-5]. If we translate this model into a modern structural formula we arrive at 1,2,4,5hexatetraene (2, biallenyl) which, of course, also possesses the sum formula $\mathrm{C}_{6} \mathrm{H}_{6}$.

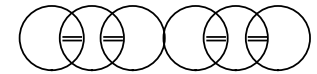

1
2

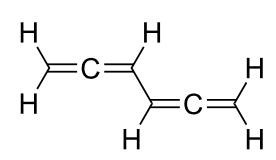

Figure 1: Loschmidt's structure proposal for benzene (1) (Scheme 181 from [3]) and the corresponding modern structural formula 2.

As it turned out, 2 was only prepared and characterized in 1970 [6], nearly 110 years after Loschmidt's and Couper's specula- tions. Still, there are some scattered reports on (conjugated) bisallenes in the chemical literature preceding the isolation of the parent system. The first "true" bisallene derivative was reported by Marvel and co-workers in the 1930s [7,8]; it is the highly substituted and hence stabilized hydrocarbon $\mathbf{3}$, a hexasubstituted derivative of $\mathbf{2}$ (Figure 2).

Twenty-five years later, Kuhn and Fischer presented the preparation of 1,1,6,6-tetraphenyl-1,2,4,5-hexatetraene (4) as the first conjugated bisallene with unsubstituted allene carbon atoms [9]. And finally, also in the 1960s, Jacobs and Prempree reported on the synthesis of the first hexa-alkylated bisallene, hydrocarbon $5[10,11]$. We shall return to the preparation of these three compounds later.

The upsurge in interest in allene chemistry is a recent phenomenon. During the 1970s (the very few) German chemists working on and with allenes used to answer inquiries about their research interests with the (German) sentence, "Ich arbeite (ueber) Allene", with allene being Berlin dialect for "alone", i.e., "I am working alone/on allenes". The international scene was not very much different: allenes and cumulenes were regarded by the chemical community as exotic compounds, of interest at best for the study of stereochemical problems (going back to the days of van't Hoff), but not as substrates and/or intermediates useful in preparative organic chemistry. Today the situation has completely changed, as is, among other things, demonstrated by numerous review articles and a recent two volumes monograph on modern allene chemistry [12-14].

As far as the organization of the present review is concerned, we have tried to cover the literature up to the end of 2011. The emphasis will be on the synthesis and the chemical transformations of bisallenes. Often bisallenes are found as side products, formed in small amounts in otherwise nonrelated reactions; these cases will not be dealt with here explicitly. The scope will be restricted to bisallenes in the strict sense of the term, i.e. we shall not be concerned with tris- and higher oligoallenes, which have also been reported occasionally. Heteroanalogues of the bisallenes such as ketenes [15] or isothiocyanates [16] will not be dealt with either, even if they contain one "intact" allene group. Also excluded are all systems in which the role of one or<smiles>CC(C)(C)C(=C=C(c1ccccc1)c1ccccc1)C(=C=C(c1ccccc1)c1ccccc1)C(C)(C)C</smiles>

3<smiles>C(=C=C=C(c1ccccc1)c1ccccc1)=C=Cc1ccccc1</smiles>

4<smiles>CC(C)=C=C(C)C(C)=C=C(C)C</smiles>

5

Figure 2: The first isolated bisallenes. 
more of the allene carbon atoms of the bisallene is taken over by metal atoms [17-19].

We will begin our discussion with the conjugated bisallenes 6 $\left(\mathrm{R}_{n}\right.$ with $n$ from 0 , i.e. H, up to 6 , fully substituted, Figure 3 ), where the substituents R can vary from alkyl and aryl to any type of functional group. For practical reasons we will not use IUPAC-nomenclature for derivatives of $\mathbf{6}$, but rather regard them as substituted conjugated bisallenes $(1,2,4,5$-hexatetraenes). In fact, we will see that bisallenes with "real" functional groups, especially substituents containing carbonyl groups, are comparatively rare.

In the next step, we "separate" the two allene moieties from each other. This can be accomplished in its simplest form by interspersing a methylene group between the two allene units $(7, n=1)$. Obviously, the larger $n$ is, the bigger the distance between the terminating allene groups. However, from a certain point on, we would not expect any interaction between the two groups anymore, making, e.g., ring-closing processes increasingly unlikely (although the reader will be astonished to see how large $n$ can be before this point is actually reached; see
Section 2.3 below). Extremely long $\alpha, \omega$-bisallenes of this type should behave just like two (connected) monoallenes. Of course, the individual methylene groups in $\mathbf{7}$ may carry functional groups.

Next we introduce unsaturated spacers ("tethers") between the two allene groups. The simplest interchange will involve a double bond, which can hold its two allene substituents in vicinal (structure 8) or geminal form, 9. Of course, a triple bond can serve as a spacer, 10, as can another allene group, 11, although we are then dealing with a trisallene [20].

An aromatic ring can play the tether role also, as demonstrated by the very simple example $\mathbf{1 2}$, as well as any other mono-, $\mathbf{1 3}$, or bi- and polycyclic ring system. In $\mathbf{1 3}$ the two allene moieties are both in exocyclic arrangement. However, both semicyclic, $\mathbf{1 4}$, and endocyclic arrangements, 15, are also conceivable. If one methylene group in $7(n=1)$ is replaced by a heteroatom, the heteroorganic bisallenes $\mathbf{1 6}$ result, in their simplest form as ethers $(X=O)$, amines $(X=N R)$, thioethers $(X=S)$, etc. Analogously bisallenic epoxides $(\mathbf{1 7}, \mathrm{X}=\mathrm{O})$ or aziridines (17, $\mathrm{X}=\mathrm{NR}$ ) can formally be generated from the corresponding all-<smiles>[R2]=C=C=C=C</smiles>

6<smiles>C=C=CC#CC=C=C</smiles>

10<smiles>C=C=C1CCCCCC1=C=C</smiles>

14<smiles>C1=CCCC=C1</smiles>

18<smiles>C=C=CCCCCCC=C=C</smiles>

7<smiles>C=C=CC=C=CC=C=C</smiles>

11

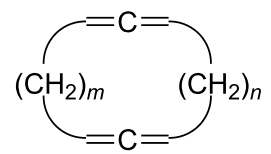

15

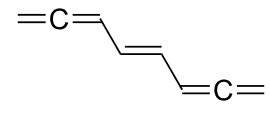

8<smiles>C=C=Cc1ccccc1C=C=C</smiles>

12<smiles>[X]C=C=C=C</smiles>

16<smiles>C=C=CC(=C)C=C=C</smiles>

9<smiles>C=C=CC1CC1C=C=C</smiles>

13<smiles>C=C=CC1C(=C=C)C1C=C=C</smiles>

17 
carbon analogues 13. Finally, we have summarized several interesting reactions in which bisallenes have been postulated as reaction intermediates; a structurally simple example to illustrate this group of compounds is 1,2,4,5-cycloheptatetraene (18), which is presumably generated as a reaction intermediate in a pyrolysis reaction (see Section 5).

How fast a very large structural variety can be generated from these basic patterns, becomes obvious if one realizes that, for example, in the aromatic systems $\mathbf{1 2}$, the allene units can be anchored also in meta- and/or para-position and condensed aromatic compounds or heteroaromatic moieties may take over the role of the aromatic core.

\section{Review}

\section{Acyclic conjugated bisallenes 1.1 Synthesis of hydrocarbons}

Bisallenes of type $\mathbf{2}$ or $\mathbf{6}$ have been prepared by many different routes and strategies from which, however, three stand out:

i) The $\mathrm{C}_{6}$-carbon framework may be constructed by adding two more carbons to a $\mathrm{C}_{4}$-precursor. Most often this route takes the form of $\mathrm{C}_{4}+\mathrm{C}_{1}+\mathrm{C}_{1}$.

ii) The $\mathrm{C}_{6}$-system is built up by coupling two $\mathrm{C}_{3}$-units $\left(\mathrm{C}_{3}+\mathrm{C}_{3}\right)$. This is probably the most versatile way to prepare substituted, conjugated bisallenes.

iii) The starting material already contains six consecutive carbon atoms, but the system is either lacking the desired substitution pattern or the proper degree of unsaturation $\left(\mathrm{C}_{6}+0\right)$.

A fourth possibility, the coupling of three $\mathrm{C}_{2}$ units $\left(\mathrm{C}_{2}+\mathrm{C}_{2}+\right.$ $\mathrm{C}_{2}$ ) is extremely rare and it appears that only two examples have been reported so far (see Scheme 12 and Scheme 17).

Starting with the simplest bisallene, the $\mathrm{C}_{6}$-isomer 1,2,4,5-hexatetraene (2), this parent system was first prepared by a protocol according to general route (ii) [6]. As summarized in Scheme 1, conversion of the $\mathrm{C}_{3}$-building block propargyl bromide (19) into its Grignard reagent, allenyl magnesium bromide (20), takes place first. The subsequent addition of $\mathrm{CuCl}$ very likely generates a copper organic intermediate, which is then coupled with a second equivalent of $\mathbf{1 9}$ to yield $\mathbf{2}$ and its isomer $\mathbf{2 1}(1,2-$ hexadien-5-yne, propargylallene). Formally, the dimerization of 19 to 2 involves two $\mathrm{S}_{\mathrm{N}} 2$ '-reactions, but detailed mechanistic experiments have not been undertaken for this particular reaction so far [21]. The two $\mathrm{C}_{6} \mathrm{H}_{6}$-isomers 2 and $\mathbf{2 1}$ are produced in ca. 2:3 ratio and the total yield varies from $40-70 \%$, depending primarily on the workup conditions. The separation of the two hydrocarbons by distillation is difficult because they are unstable und possess similar physical constants. If pure $\mathbf{2 1}$ were needed, 2 can be removed from the reaction mixture by trapping it by a Diels-Alder reaction (see Section 1.4.1 below). The analytically pure hydrocarbons were obtained by preparative gas chromatography [6]. Solutions of $\mathbf{2 / 2 1}$ in ether containing up to $50 \mathrm{~g}$ of the former can be prepared easily [21].

In principle this coupling reaction can also be employed to prepare the 1- and 3-monomethyl derivatives of 2 [22]. For preparative applications this approach is unsatisfactory, however, since too many isomers and secondary products are produced and their separation is difficult. Nevertheless, to determine the spectroscopic properties of these $\mathrm{C}_{7} \mathrm{H}_{8}$-hydrocarbons, they can be (and have been) separated by preparative gas chromatography.

In preparative chemistry one of the most frequently used conjugated bisallenes is the 1,1,6,6-tetramethyl derivative of $\mathbf{2}$, 24 (Scheme 2), which was synthesized conveniently by Skattebøl and co-workers by a route that was later termed the Doering-Moore-Skattebøl synthesis of allenes (DMS-synthesis) [23], i.e., a $\mathrm{C}_{4}+\mathrm{C}_{1}+\mathrm{C}_{1}$-route belonging to category (i). In this synthesis the tetramethylbutadiene $\mathbf{2 2}$ is first dibromocyclopropanated to the tetrabromide $\mathbf{2 3}$, which, on treatment with an organolithium reagent, is debrominated/rearranged to the target hydrocarbon 24. Hydrocarbon $\mathbf{2 4}$ is a stable colorless solid that can be worked with under normal laboratory conditions without any problems.

The partially deuterated derivatives $\mathbf{3 1}$ and $\mathbf{3 2}$ have also been obtained by a $\mathrm{C}_{3}$-dimerization route (category (ii), see above), as shown in Scheme 3 [24]. 3,3-Dimethylpropargyl alcohol (25) was first converted by standard methodology to the deuterioallene 27 via the deuterioacetylene 26. Metalation of $\mathbf{2 7}$ with $n$-butyllithium provided the allenyllithium reagent 28 next, which was subsequently converted into the organozinc reagent

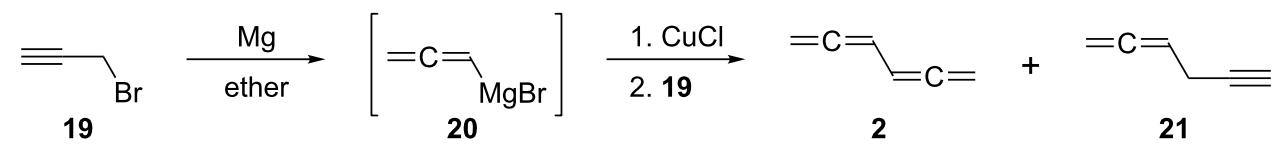

Scheme 1: The preparation of 1,2,4,5-hexatetraene (2). 


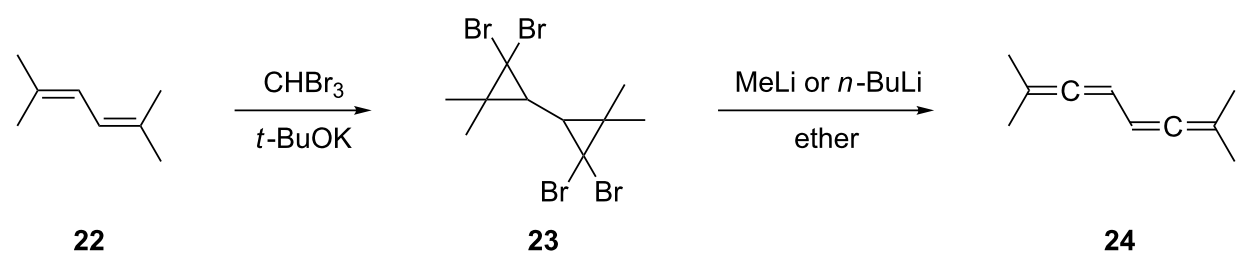

Scheme 2: The preparation of a conjugated bisallene by the DMS-protocol.

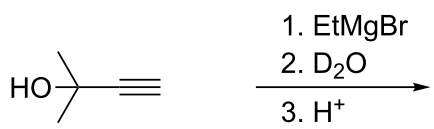

25

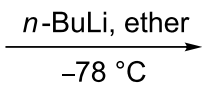

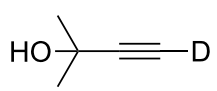

26
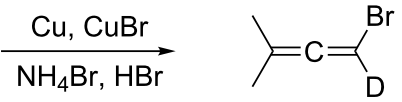

27<smiles>[2H]C([AlH2])=C=C(C)C</smiles>

28

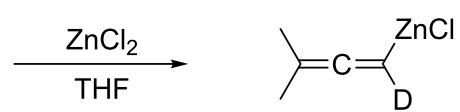

29

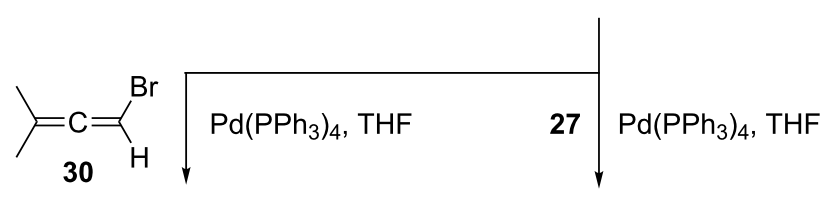<smiles>[2H]C(=C=C(C)C)C=C=C(C)C</smiles>

31<smiles>[2H]C(=C=C(C)C)C([2H])=C(C)C</smiles>

32

Scheme 3: Preparation of the 3-deuterio- and 3,4-dideuterio derivatives of 24.

29. In a Negishi-type coupling of this intermediate with either the bromoallene $\mathbf{3 0}$ or its deuterated version $\mathbf{2 7}$ the two target compounds were obtained in the final step. Neither the 3 -deuterio nor the 3,4-dideuterio derivative of the parent system 2 seem to be known.

Applying the DMS-approach to either isoprene or 1,3-pentadiene leads to the desired monomethyl derivatives of $\mathbf{2}$, 3-methyl-1,2,4,5-hexatetraene and 1,2,4,5-heptatetraene, but the yields are poor and fulvenes are produced as side products [25]. The formation of fulvene derivatives and other compounds containing five-membered rings has often been noted in this type of approach [26]. These cyclic products are formed by mechanisms involving carbene (generated during the dehalogenation step of the dibromocyclopropane precursors) to carbene rearrangements followed by hydrogen shifts.

The above $\mathrm{C}_{3}$-dimerization pathway (Scheme 3) via organozinc compounds goes back to Vermeer et al. and appears to be not only the most general route to alkylated conjugated bisallenes
[27-31], but also to many other highly substituted allenes; it is summarized in compact form in Scheme 4. By this approach the substituted allene derivatives $\mathbf{3 3}$ or the propargyl derivatives 34 are coupled with organometallic reagents $\mathbf{3 5}$ in the presence of a Pd-catalyst to the different allenes and bisallenes 36 . The yields are normally good to excellent and the workup does not require chromatography of any kind.

Many other $\left(\mathrm{C}_{3}+\mathrm{C}_{3}\right)$-couplings have been reported in the chemical literature. For example, 2-butynyl halides 37 on treatment with Grignard reagents $\mathrm{RMgBr}$ provide hydrocarbons such as 3,4-dimethyl-1,2,4,5-hexatetraene (38), but the composition of the product mixture is anything but simple (Scheme 5) [32-36]. Not only are the three isomeric $\mathrm{C}_{3}$-coupling products $\mathbf{3 8}$ to $\mathbf{4 0}$ produced, but also those of $\mathbf{3 7}$ with the Grignard reagent, 41 and $42(\mathrm{R}=\mathrm{Et}, n$-Pr). All of these $\mathrm{C}-\mathrm{C}$-coupling reactions are thought to occur via radical intermediates. The potential of the coupling of 2-alkynylboranes with propargyl bromides to furnish alkylated bisallenes has evidently not been exploited yet [37]. 
<smiles>[X]C=C=C([R])[R]</smiles>

or

33
$\mathrm{R}^{1}, \mathrm{R}^{2}=\mathrm{H}, \mathrm{H} ; \mathrm{Me}, \mathrm{H}$; $\mathrm{Me}, \mathrm{Me}$ $\mathrm{X}=\mathrm{Br}, \mathrm{OAC}$

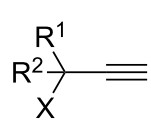

34

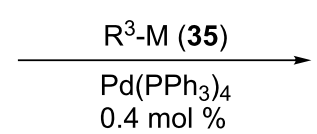

$0.4 \mathrm{~mol} \%$<smiles>[R]C=C=C([R])[R]</smiles>

36

$\mathrm{R}^{1}, \mathrm{R}^{2}=\mathrm{H}, \mathrm{H} ; \mathrm{Me}, \mathrm{H} ; n-\mathrm{C}_{5} \mathrm{H}_{11}, \mathrm{H}$; $t-\mathrm{Bu}, \mathrm{H} ; \mathrm{Me}, \mathrm{Me} ; \mathrm{iPr}, \mathrm{Me}$ $\mathrm{X}=\mathrm{Br}, \mathrm{OAc}, \mathrm{O}(\mathrm{SO}) \mathrm{Me}$, $\mathrm{OSO}_{2} \mathrm{Me}, \mathrm{OP}(\mathrm{O})(\mathrm{OEt})_{2}$<smiles>[X]CC#CC[18O]</smiles>

$(\mathrm{X}=\mathrm{Cl}, \mathrm{Br}, \mathrm{I})$<smiles>C=C=C(C)C(C)=C=C</smiles>

38

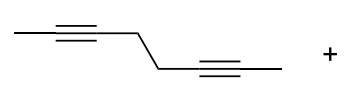

40
$\mathrm{R}^{3}=\widehat{\gamma}, \quad \mathrm{TMS}=\xi$<smiles>C=C([Tl])CC(=C)CI</smiles><smiles>C#CC#CI</smiles>

$t-\mathrm{Bu}-\mathrm{CH}=\mathrm{C}=\mathrm{CH}-\xi$

$X=\mathrm{Li}, \mathrm{MgCl}, \mathrm{Cu}, \mathrm{Cu}_{1 / 2} \mathrm{Li}_{1 / 2}$,

$\mathrm{Ag}, \mathrm{Ag}_{1 / 2} \mathrm{Li}_{1 / 2}, \mathrm{ZnCl}, \mathrm{Zn}_{1 / 2}$

Scheme 5: A preparation of 3,4-dimethyl-1,2,4,5-hexatetraene (38).

The last general approach (see (iii) above), in which the substrate molecule already contains all the carbon atoms required in the product, has also been employed for the preparation of the parent hydrocarbon 2 (Scheme 6). The reduction of 1,6-dichloro-2,4-butadiyne (43) in methanol with a zinc/copper couple furnished the bisallene 2 , but also provided at least three other $\mathrm{C}_{6} \mathrm{H}_{6}$-isomers, 44-46, requiring extensive chromatographic separation and purification [38]. Biallenyl $\mathbf{2}$ is also produced when 1,5-hexadiyne is treated with sodium ethoxide in ethanol at $65^{\circ} \mathrm{C}$ for $24 \mathrm{~h}$. But again this is not a practical preparative method since it results in the formation of too many other isomers of the substrate and 2 [39].

Alkylated and terminally cycloalkylated bisallenes have also been obtained by another approach starting from $\mathrm{C}_{6}$-derived precursors: the alkylation of diacetylene glycol esters with organoaluminum reagents. The example shown in Scheme 7 is typical. Treatment of diacetate 47 with triethylaluminum in ether under reflux for $4 \mathrm{~h}$ provides the fully alkylated bisallene $\mathbf{4 8}$ in $56 \%$ yield $[40,41]$.

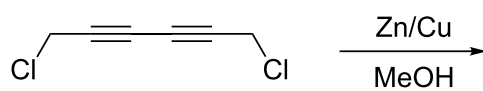

43<smiles>C=C=CC=C</smiles>

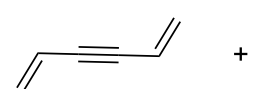

44

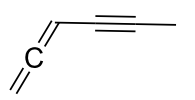

45

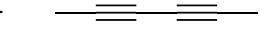

46

Scheme 6: A $\left(C_{6}+0\right)$-approach to 1,2,4,5-hexatetraene (2).

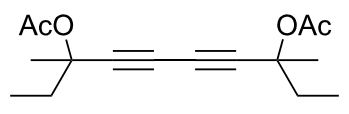

47
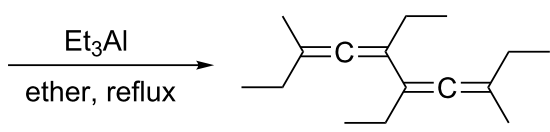

$48(56 \%)$ 
Turning to phenyl- and/or aryl-substituted conjugated bisallenes, we have already encountered two classic representatives of this subclass: hydrocarbons $\mathbf{3}$ and $\mathbf{4}$ (see above). Both were obtained by general method (iii). In Marvel's route to 3 [7,8] the bispropargyl precursor $\mathbf{4 9}$ was thermally isomerized to the bisallene (Scheme 8 ) by exploiting a process to which we shall return later (see Section 1.4.1). In Kuhn's synthesis of $\mathbf{4}$ the [5]cumulene $\mathbf{5 0}$ was reduced in good yield to the conjugated bisallene by treatment with aluminum amalgam under very mild conditions [9].

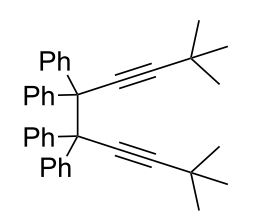

49

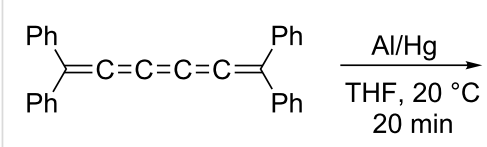

50

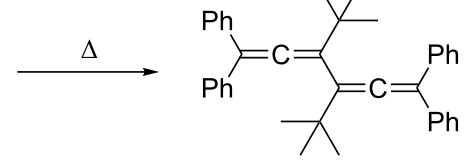

3

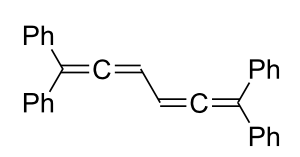

$4(75 \%)$
Scheme 8: The preparation of the first phenyl-substituted conjugated bisallenes 3 and 4 .

In an approach related to Kuhn's reduction of $\mathbf{5 0}$ to $\mathbf{4}$, tetraalkyl[5]cumulenes $\mathbf{5 1}$ are selectively hydrogenated with $\mathrm{Zn} / \mathrm{ZnCl}_{2}$ in aqueous ethanol (Scheme 9) to the conjugated bisallenes 52, again fully substituted in their terminal positions. The yields are almost quantitative (88-97\%) and, provided the substrates $\mathbf{5 1}$ are available by a straightforward method, this synthesis can probably be extended [42].

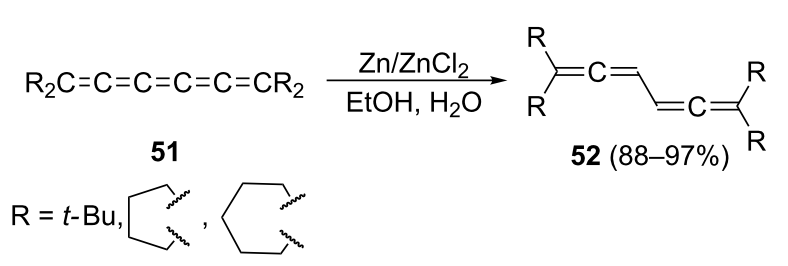

Scheme 9: Selective hydrogenation of [5]cumulenes to conjugated bisallenes: another $\left(\mathrm{C}_{6}+0\right)$-route.
Not surprisingly the $\mathrm{C}_{3}$-dimerization protocol works for arylsubstituted bisallenes also as shown, for example, in Scheme 10 [43]. Thus, treatment of the phenylpropargyl alcohol $\mathbf{5 3}$ with $\mathrm{TiCl}_{3} / \mathrm{CH}_{3} \mathrm{Li}$ leads to the 1,6-diphenylbiallenyl 54. Not only is the process characterized by a poor yield, but it also provides two isomers $\mathbf{5 5}$ and $\mathbf{5 6}$ of $\mathbf{5 4}$ as well as the deoxygenated (and rearranged) substrate 57. Clearly, this is not a preparative method, but was sufficient to produce spectroscopic amounts of the desired hydrocarbon.

Better results were obtained by Toda and Takehira, who dimerized the 1,1-diphenylallenyl bromide $\mathbf{5 8}$ with $\mathrm{CuCl}$ in $\mathrm{DMF}$ at room temperature in good yield to hexaphenylbisallene $\mathbf{5 9}$ (Scheme 11) [44]. The reaction could also be applied to the synthesis of other arylated bisallenes and presumably takes place via radical intermediates.<smiles>BrC(=C=C(c1ccccc1)c1ccccc1)c1ccccc1</smiles>

58<smiles>C(=C(C(=Cc1ccccc1)c1ccccc1)c1ccccc1)=C(c1ccccc1)c1ccccc1</smiles>

$59(72 \%)$
Scheme 11: Hexaphenyl-1,2,4,5-hexatetraene (59) by a $\left(\mathrm{C}_{3}+\mathrm{C}_{3}\right)$-approach.

A more recent way to allenes exploits the allenation of carbonyl compounds with titanocene alkenylidene reagents such as $\mathbf{6 0}$. When applied to bibenzoyl (benzil), 61, the 3,4-diphenylhexatetraene $\mathbf{6 2}$ is produced in good yield as the sole product (Scheme 12) [45]. Formally this constitutes a $\left(\mathrm{C}_{2}+\mathrm{C}_{2}+\mathrm{C}_{2}\right)$ route (see also Scheme 17).<smiles>C=C=C(C(=C=C)c1ccccc1)C(=C=C)c1ccccc1</smiles>

Scheme 12: An allenation route to conjugated bisallenes.

We will encounter many of these routes to conjugated bisallenes in the coming sections dedicated to the preparation of<smiles>C#CC(O)c1ccccc1</smiles>

53<smiles>C(=C=C=Cc1ccccc1)=C=Cc1ccccc1</smiles>

$54(8 \%)$<smiles>C#CC(C=C=Cc1ccccc1)c1ccccc1</smiles>

$55(10 \%)$<smiles>C#CC(c1ccccc1)C(c1ccccc1)c1ccccc1</smiles> 
compound types $\mathbf{7 - 1 7}$. Not surprisingly, though, we will also meet new and specific approaches, and these will be discussed in due course.

\subsection{Synthesis of functionalized systems}

Derivatives of $\mathbf{2}$ carrying "real", i.e. heteroatom-containing functional groups, are comparatively rare and this constitutes one serious drawback of using these electron-rich compounds in preparative organic chemistry. For example, a systematic substructure search for conjugated bisallenes has revealed that virtually nothing is known about tetraolefins of this type carrying any carbonyl-containing function.

As far as halogen substituents are concerned the situation is slightly better (see below). An early reference to the preparation of 3,4-dichloro-1,2,4,5-hexatetraene by Carothers and Coffman requires reinvestigation with modern spectroscopic methods $[46,47]$. If confirmed, it would not only describe the first preparation of a functionalized bisallene, but also the first representative of this class of molecules at all. Furthermore, by employing this dichloride in modern, metal-mediated coupling reactions it could become a useful substrate for the synthesis of highly unsaturated hydrocarbons, in particular of cross-conjugated systems (dendralenes) [48]. In fact, the only reaction coming close to a general route to introduce functionality into bisallenes at the present time seems to be the double $\mathrm{S}_{\mathrm{N}} 2^{\prime}$-reaction of 2,4-hexadiyne-1,6-diol (63, e.g. $\mathrm{R}^{1}$ to $\mathrm{R}^{4}=$ aryl) and its derivatives with various nucleophiles (Scheme 13) $[49,50]$. Thus, heating the diols $\mathbf{6 3}$ with an aromatic or heteroaromatic thiol 64 provides the sulfur-substituted bisallene derivative $\mathbf{6 5}$ in good yields. Likewise, the stable diallenes $\mathbf{6 6}$ carrying phosphorus-containing substituents were obtained by heating the diols 63 with $\mathrm{R}_{2}^{5} \mathrm{PCl}$ [51-54].

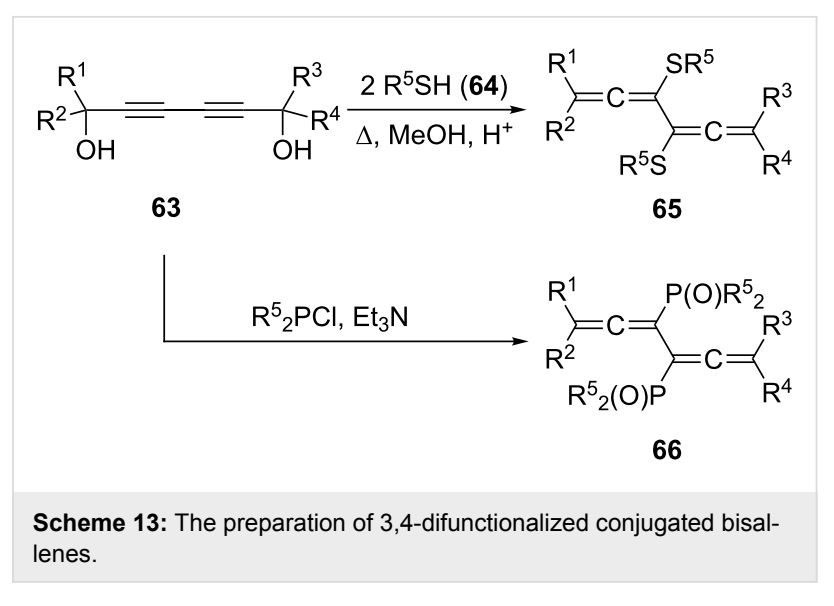

How critically the substituents in the 3- and 4-position determine the stability and reactivity of conjugated bisallenes was shown by treating the tetramethyl derivative $\mathbf{6 7}$ and related compounds with $\mathrm{Cl}_{3} \mathrm{CSCl}$ in triethylamine (Scheme 14) [55]. Although the bisallenes 68 could be identified unequivocally by spectroscopic methods at low temperatures, isolation of these compounds at room temperature failed because they easily isomerized to the dienynes $\mathbf{6 9}$. The derivatives $\mathbf{7 1}$ can also be obtained from the isomeric diols $\mathbf{7 0}$ under comparable conditions, as shown by Braverman and co-workers. However, in only one case could these bisallene derivatives actually be isolated; all other compounds isomerized to the 3,4-bismethylenecyclobutene derivatives $\mathbf{7 2}$ by an electrocyclic process to which we shall return later (Section 1.4.1) [56,57].

In fact all of these reactions were preceded by the work of Bohlmann and Kieslich, who in the 1950s already prepared various 3,4-dibromobisallenes $\mathbf{7 4}$ by treating the highly substituted diyne diols $\mathbf{7 3}$ with phosphorus tribromide in benzene

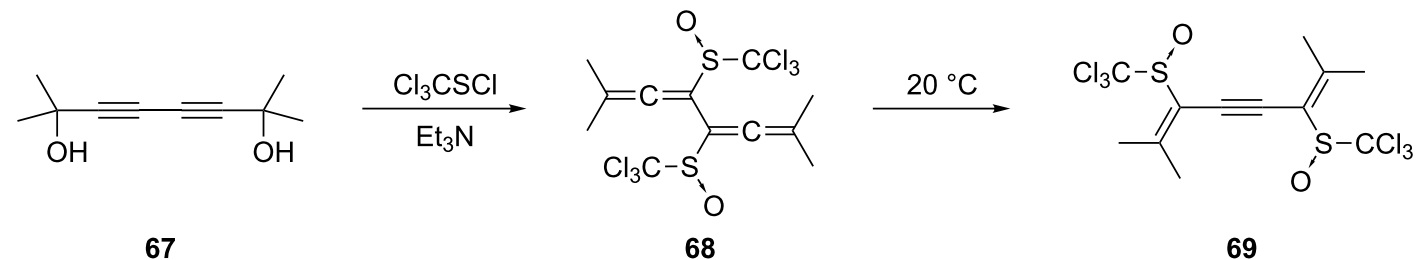

67

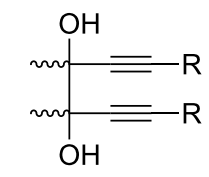

$70(\mathrm{R}=\mathrm{H}, \mathrm{Ph})$

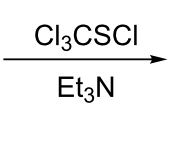

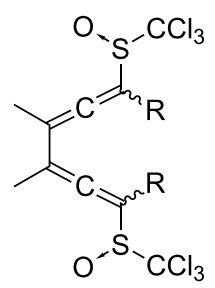

71<smiles>[R]C(=C1C(C)=C(C)C1=S(O)C(Cl)(Cl)Cl)[SH](O)C(Cl)(Cl)Cl</smiles>

72 
(Scheme 15) [58-63]. The details of these often overlooked seminal publications were recently studied by Parkhurst and Swager. According to these authors the 1,6-dibromide is produced first from the 1,6-diynediol. It isomerizes to the 3,4-dibromobisallene derivative next, especially under the influence of $\mathrm{CuBr}$. The bisallene can subsequently undergo a $\mathrm{CuBr}$ induced cyclization to a 1,2-dibromo-3,4-bismethylenecyclobutene derivative (see Section 1.4.1), thus providing a convenient route to these preparatively interesting four-membered ring compounds [64].
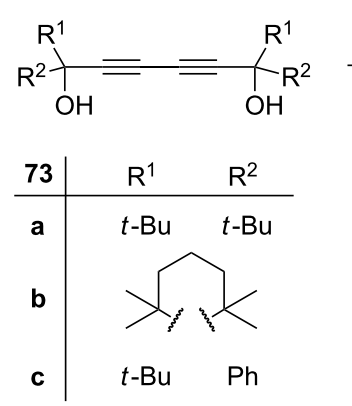

Scheme 15: The preparation of 3,4-dibromo bisallenes.

When an allene carries a hydroxy function, it is, of course, a special type of an enol (an allenol) and as such unstable. Yet, it may be generated as an intermediate to be employed in further transformations. Indeed, when $\alpha$-diketones such as benzil (61, 1,2-diphenyl-1,2-ethanedione), are treated with two equivalents of a metal acetylide, the bisalcoholate $\mathbf{7 5}$ is produced first (Scheme 16) [65]. The expected [3.3]sigmatropic rearrangement of 75 takes place readily (see below, Section 1.4.1) at room temperature (it is, in essence, a double oxy-Cope rearrangement), and the resulting $\mathbf{7 6}$ subsequently undergoes an electrocylic ring closure to a four-membered ring system. On workup (hydrolysis) a mixture of the cis and trans isomers of 77 is obtained in very good yield (ca. 80\%).

$2 \mathrm{R}^{1}=\mathrm{Li}+\mathrm{Ph}_{\mathrm{O}}^{\stackrel{\mathrm{O}}{=}} \mathrm{Ph} \stackrel{-78^{\circ} \mathrm{C}}{\longrightarrow}$

61

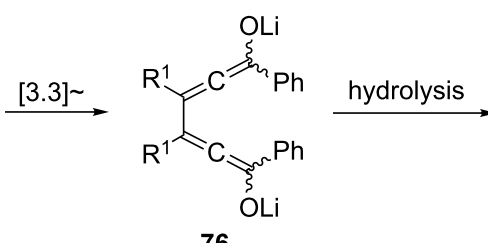

76<smiles>[R]C#CC([Ge])(c1ccccc1)C(Cl)(C#[R])C(Cl)(Cl)c1ccccc1</smiles>
75

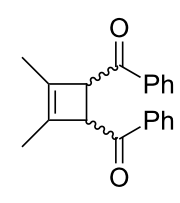

77 (80\%)

Scheme 16: Generation of allenolates by an oxy-Cope rearrangement
A conjugated bisallene carrying three diphenylphosphine substituents has been prepared recently by a Zr-promoted linear coupling of different aryl and hetaryl acetylenes in high yield (Scheme 17) [66]. Treating, e.g., phenylacetylene (78) first with $n$-butyllithium and subsequently with bis(cyclopentadienyl)zirconium dichloride generates the trimeric metal complex 79, which on quenching with diphenylchlorophosphine yields the conjugated bisallene $\mathbf{8 0}$ in practically quantitative yield. Analogously, on quenching with trimethylsilyl triflate, 79 furnishes the tris(trimethylsilyl) derivative 81. This formal $\left(\mathrm{C}_{2}\right.$ $+\mathrm{C}_{2}+\mathrm{C}_{2}$ )-approach constitutes the only direct example we could find in the chemical literature for building up a bisallene derivative by a trimerization protocol from the same $\mathrm{C}_{2}$-precursor (acetylene).

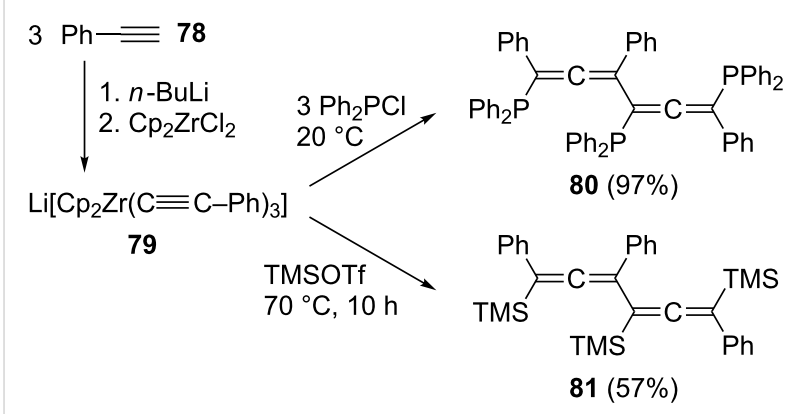

Scheme 17: A linear trimerization of alkynes to conjugated bisallenes: a $\left(\mathrm{C}_{2}+\mathrm{C}_{2}+\mathrm{C}_{2}\right)$-protocol.

Many other silylated derivatives of $\mathbf{2}$ have been described. For example, derivative $\mathbf{8 3}$ can be obtained in good yield by a $\mathrm{C}_{3}$-coupling route, as demonstrated in Scheme 18, from a trimethylsilyl-protected propargyl alcohol, 82 [67].

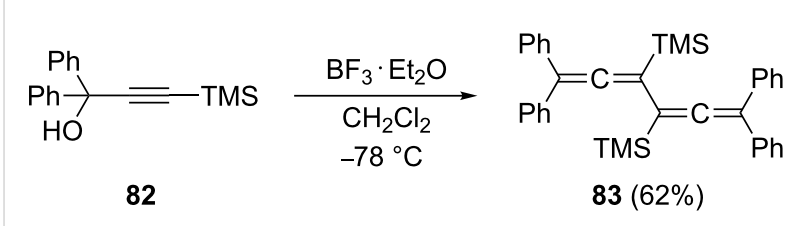

Scheme 18: Preparation of a TMS-substituted conjugated bisallene by a $\mathrm{C}_{3}$-dimerization route.

The fully substituted bisallene with two internal trimethylsilyl substituents 86 was prepared by McGlinchey et al. by a $(2 \times$ $\mathrm{C}_{3}$ )-coupling route from the bromoallene 84 (Scheme 19) [68]. When $\mathbf{8 4}$ was heated with $n$-butyllithium in THF it dimerized to the unusual propargylallene derivative $\mathbf{8 5}$, which, on slight heating $\left(65^{\circ} \mathrm{C}\right)$, isomerized to the bisallene 86 , which exists in the shown transoid conformation. On heating this conjugated bisallene to $110{ }^{\circ} \mathrm{C}$ it undergoes a remarkable isomerization/ dimerization reaction to which we shall return in Section 1.4.1. 


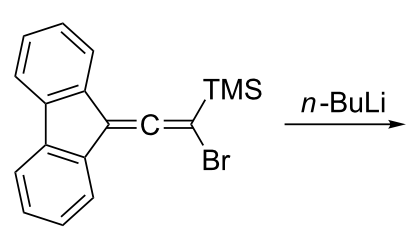

84

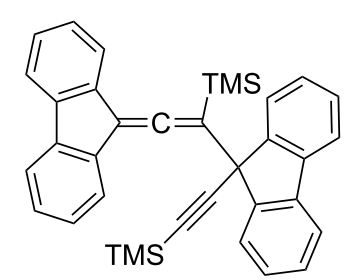

85

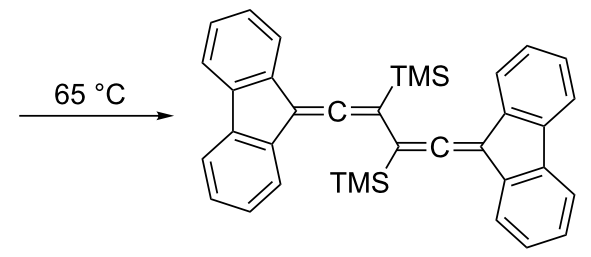

86
Scheme 19: A bis(trimethylsilyl)bisallene by a $C_{3}$-coupling protocol.

The fully trimethylsilylated bisallene $\mathbf{8 8}$ is produced when hexabromide $\mathbf{8 7}$ is treated with excess trimethylsilyl chloride and Rieke magnesium at $0{ }^{\circ} \mathrm{C}$ (Scheme 20) [69,70]. The yield is low, however, and several other isomers of $\mathbf{8 8}$ are present in the reaction mixture. A far superior route to $\mathbf{8 8}$ consists of the thermal isomerization of hexakis(trimethylsilyl)benzene (89) $[71,72]$. Under flash vacuum pyrolysis conditions $\left(400{ }^{\circ} \mathrm{C}\right.$, $<0.01$ Tor) $\mathbf{8 8}$ is produced from $\mathbf{8 9}$ as a mixture with several of its isomers (see below), but heating of the aromatic substrate at $200{ }^{\circ} \mathrm{C}$ for $10 \mathrm{~h}$ provides the bisallene as the sole product.

To rationalize the deep-seated reshuffling of the six carbon atoms of the benzene ring, Sakurai and co-workers suggested the mechanism given in Scheme 21. In the first step, the starting material 89, which possesses a distorted chair structure, undergoes a TMS-shift to provide the diradical intermediate 90. In the next step the ring is split to furnish derivative 91, which, by another TMS-shift, isomerizes to the propargylallene $\mathbf{9 2}$. The sequence ends with still another TMS-relocation to provide the final product $\mathbf{8 8}$. Both, 91 and $\mathbf{9 2}$ are pyrolysis products under flash vacuum conditions (see above).

Interestingly, there are several other observations demonstrating that an extended network of relations exists between many of these $\mathrm{C}_{6}$-carbon frameworks. For example, hexakis(trimethylsilyl)-3,3'-bicyclopropenyl (93), upon either sensitized irradiation with a mercury-arc lamp or heating ( $250{ }^{\circ} \mathrm{C}$, benzene), is converted quantitatively to $\mathbf{8 8}$, which is presumably the thermodynamically most stable of the $\mathrm{C}_{6}$ (TMS) ${ }_{6}$-isomers (Scheme 22) [73]. West and Priesner obtained $\mathbf{8 8}$ as one of the products produced when 2,4-hexadiyne was first metalated by the $n$-butyllithium/TMEDA-complex at room temperature and the resulting trilithiated compound $\mathrm{MeC}_{5} \mathrm{Li}_{3}$ subsequently trapped with trimethylsilyl chlo-

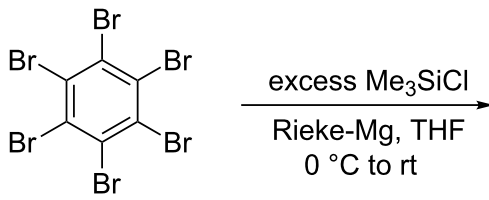

87<smiles>CC(C)=C=C(C)C(=C=C(C)S(C)(=O)=O)C([As])=C(C)S(C)(=O)=O</smiles>

88<smiles>Cc1c([As])c(C(C)(C)C)c(S(C)(=O)=O)c(S(C)(=O)=O)c1S(C)(=O)=O</smiles>

89

Scheme 20: The rearrangement of highly substituted benzene derivatives into their conjugated bisallenic isomers.<smiles>CC(C)(C)c1c(S(C)(=O)=O)c([As])c(S(C)(=O)=O)c(S(C)(=O)=O)c1S(C)(=O)=O</smiles>

89

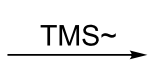

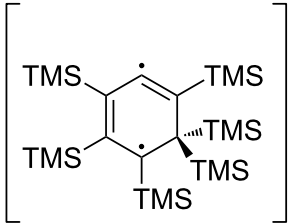

90<smiles>CC#CC(C)=C(C(C)=S)C(C)=C(C)[As]</smiles><smiles>CC#CC(C)(C)C(C)=C(C(C)C)C(C)(C)C</smiles>

92<smiles>CC(=C=C(C(=C(C)C)C(C)(C)C)C(C)(C)C)S(C)(=O)=O</smiles>

88 
ride [74]. Comparable studies had already been carried out by Klein and Becker several years earlier [75].

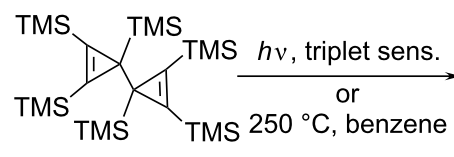

93

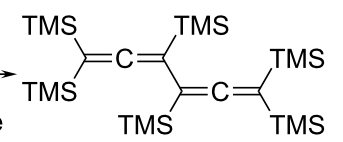

88
Scheme 22: From a bicyclopropenyl to a conjugated bisallene derivative.

Still another isomer of benzene is 3,4-bismethylenecyclobutene. When its hexachloro derivative $\mathbf{9 4}$ was treated by Gilman and co-workers with excess dimethylsilyl chloride and magnesium in THF, the hexasilylated derivative $\mathbf{9 5}$ was produced and again a ring-opening reaction to a conjugated bisallene had taken place (Scheme 23) [70]. In closing this section on silicon-substituted conjugated bisallenes, it should be mentioned that both the hexakis(trichlorosilyl) [76] and the hexakis(trimethylgermyl) [77] analogues of $\mathbf{8 8}$ and $\mathbf{9 5}$ are known.
The number of mono- and difunctionalized derivatives of $\mathbf{2}$ is still rather small. A case in point was provided by Goré and co-workers who obtained the primary and secondary alcohols 99 by first coupling the enynol 96 with different propargyl chlorides 97 to the allenynols 98 (Scheme 24) [78,79]. These latter derivatives were then reduced by either $\mathrm{LiAlH}_{4}$ or $\mathrm{LiAlH}_{3}\left(\mathrm{OCH}_{3}\right)$ to provide the substituted bisallenes $\mathbf{9 9}$ in yields between 50 and $75 \%$.

Structurally comparable bisallene diols could be prepared, as shown by Krause and Poonoth [80,81], from bisoxiranes $\mathbf{1 0 0}$ by opening these with Grignard reagents in the presence of copper salts; this is again a double $\mathrm{S}_{\mathrm{N}} 2$ '-process (Scheme 25). The resulting derivatives $\mathbf{1 0 1}$ are useful precursors for a double cyclization to novel bis(2,5-dihydrofuran) derivatives 102; the yields in all three steps are acceptable to good (up to 60\%).

A protected biallenyl diol, the derivative 104, is produced as a mixture of diastereomers in small amounts $(10 \%)$ when the mesylate $\mathbf{1 0 3}$ is treated with $\mathrm{Pd}\left(\mathrm{PPh}_{3}\right)_{4}$ and diethyl zinc in THF at $0{ }^{\circ} \mathrm{C}$ (Scheme 26; OTBDPS = tert-butyldiphenylsilyloxy)<smiles>ClC(Cl)=C1C(Cl)=C(Cl)C1=C(Cl)Cl</smiles>

94 $\underset{20 \text { equiv } \mathrm{Mg}, \mathrm{THF}}{\stackrel{10 \text { equiv } \mathrm{Me}_{2} \mathrm{SiHCl}}{\longrightarrow}}$

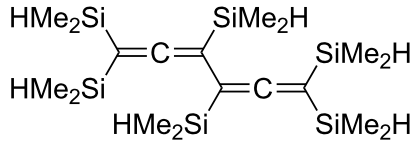

95

Scheme 23: The conversion of a bismethylenecyclobutene into a conjugated bisallene.<smiles>[R]C(C#C)=C([R])C([R])O</smiles>

96 $\mathrm{R}^{1}-\mathrm{R}^{3}: \mathrm{H}, \mathrm{CH}_{3}$; $\mathrm{R}^{4}: \mathrm{H}, \mathrm{OCH}_{3}$<smiles>[R]C([R7])(Cl)C#C</smiles>

97<smiles>[R]C([R])=C=CC#CC([R])=C([R])[R]</smiles>

98<smiles>[R8]C([R])=C=CC=C=C([R])C([R])O</smiles>

$99(50-75 \%)$

Scheme 24: The preparation of monofunctionalized bisallenes.<smiles>[R20]CC1OC1(C)C#CC#CC1(C)OC1CO[R2]</smiles>
100

\section{$\underset{\mathrm{CuBr} \cdot \mathrm{Me}_{2} \mathrm{~S}}{\stackrel{\mathrm{MgCl}}{\mathrm{LiBr}}}$ THF \\ $-65^{\circ} \mathrm{C}$ to rt}<smiles>[R20]CC(O)/C(C)=C\C([R])=C([R])\C=C(/C)C(O)C[R20]</smiles>

Au or Ag salts<smiles>[R10]OCC1OC([R])(C2([R])C=C(C)C(CO[R10])O2)C=C1C</smiles>

102 
[82]. The allenic dimer is presumably formed by coupling of a transient allenyl zinc intermediate with an initially generated allenyl palladium species.

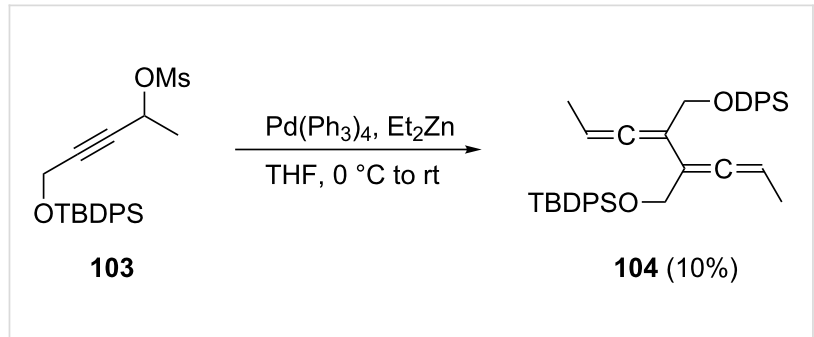

Scheme 26: A 3,4-difunctionalized conjugated bisallene by a $\mathrm{C}_{3}$-coupling process.

Another type of a difunctionalized conjugated bisallene has been produced by a coupling reaction. In this case the diketone 107 was obtained by a $\mathrm{Zn} / \mathrm{Cu}$-couple-induced coupling reaction between excess phenacyl bromide (105) and the enyne $\mathbf{1 0 6}$ (Scheme 27) [83]. Besides 107 its diyne and propargylallene dimers were produced, requiring a chromatographic separation of the product mixture. The overall yields were very good (78-91\%); the relative yields of the three dimers were not determined, however.

A monosubstituted bisallene has been prepared by Braverman and co-workers by treatment of the sulfonium or selenium salt $\mathbf{1 0 8}$ with DBU in acetone at $0{ }^{\circ} \mathrm{C}$, providing a mixture of $\mathbf{1 0 9}$ and $\mathbf{1 1 0}$ in a 7:3 ratio (Scheme 28) [84]. For the formation of $\mathbf{1 1 0}$ a [2.3]sigmatropic rearrangement of $\mathbf{1 0 8}$ has been suggested, with the intermediately generated propargylallene derivative being stabilized by a prototropic shift to the final product $\mathbf{1 1 0 .}$

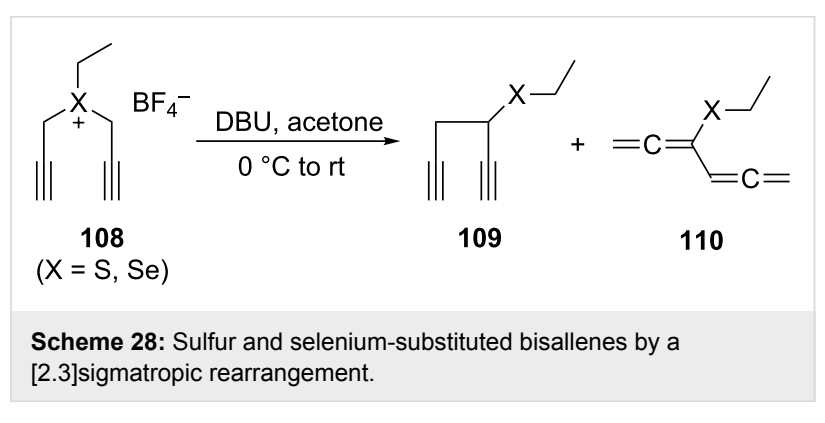

Another route to an unusually functionalized conjugated bisallene was reported recently [85]. The reaction amounts to a 1,2,4,5-hexatetraenylation of 4-acetoxy-2-azetidinones, such as 111 (Scheme 29). In this case the organoindium reagent, formed in situ from 1,6-dibromo-2,4-hexadiyne (112) and indium, was coupled with 111 in the presence of $\mathrm{LiCl}$ in DMF to yield the 2-azetidinone 113 carrying the 1,2,4,5-hexatetraen-3-yl substituent in the 4-position. The bisallene is a very good diene for Diels-Alder cycloadditions as will be discussed in Section 1.4.1.

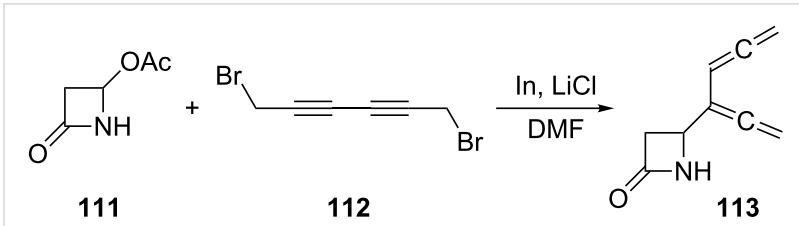

Scheme 29: The biallenylation of azetidinones.

The unusual hexakis(ferrocenyl)-1,2,4,5-hexatetraene (116) is produced as one of many side-products when tris(ferrocenyl)allenylium tetrafluoroborate (114) is treated with 1-cuprioferrocene (115) (Scheme 30) [86].<smiles>[R]C#CC(=C)C</smiles>

Scheme 27: Preparation of a bisallenic diketone by a coupling reaction.

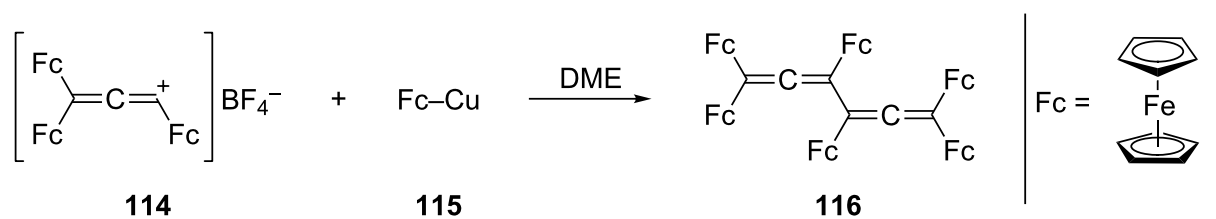


A number of bisallenic systems have been described in which the conjugated framework carries acetylenic substituents. In fact the earliest reports on these compounds, from Marvel and co-workers [87], go back to the 1930s (Scheme 31). Again, a thermal isomerization of a 1,5-hexadiyne derivative to a conjugated bisallene was employed, a process that will be discussed in detail in Section 1.4.1. Hexakis(tert-butylethynyl)ethane (117), when heated in alcoholic solution, isomerizes to the diallene 118.

Several hydrocarbon systems with a smaller number of triple bonds than $\mathbf{1 1 8}$ have also been reported. For example, Toda and Takahara have prepared the allenyne bromide 121 from the ester 119 via $\mathbf{1 2 0}$ by routine transformations (Scheme 32) [88,89]. When $\mathbf{1 2 1}$ is oxidatively coupled, e.g. a $\left(\mathrm{C}_{3}+\mathrm{C}_{3}\right)$-approach to bisallenes is employed (see above), three dimers are obtained: the $d$,l-bisallene $\mathbf{1 2 2}$, the meso-diastereomer 123 and the propargylallene 124. We will discuss the remarkable thermal behavior of $\mathbf{1 2 2}$ and 123 in Section 1.4.1. A bisallene derivative carrying two alkynyl substituents at its inner (C-3 and C-4) positions is presumably formed when 1,1,2,2-tetrakis(tertbutylethynyl)ethane is first treated with phenyllithium and the resulting dianion is subsequently quenched with water [90].

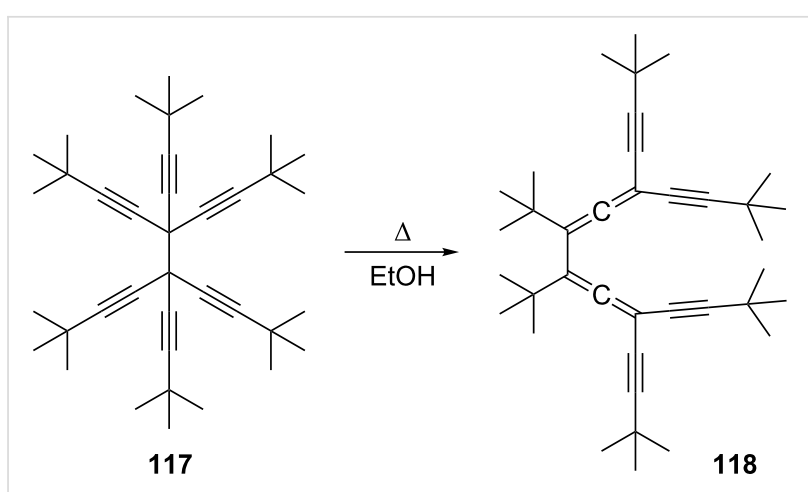

Scheme 31: The first isomerization of a 1,5-hexadiyne to a 1,2,4,5hexatetraene.

Finally, it has been suggested that the diacetylenic bisallene 127, carrying six ferrocenyl substituents (see Scheme 30) is produced when the diacetylene $\mathbf{1 2 5}$ is first ionized with $\mathrm{HBF}_{4}$ and the thus generated cation $\mathbf{1 2 6}$ reductively dimerized to $\mathbf{1 2 7}$ by treatment with FcLi and $\mathrm{CuBr} / \mathrm{Me}_{2} \mathrm{~S}$ in DME (Scheme 33) [91].

\subsection{Spectroscopic and structural properties of conju- gated bisallenes}

None of the numerous conjugated bisallenes discussed in Section 1.1 have been investigated from the structural and spec-

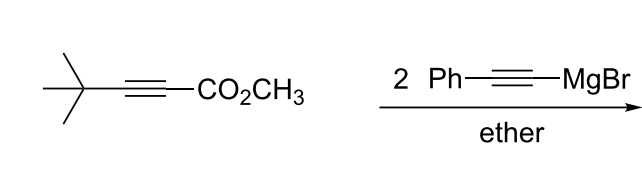

119

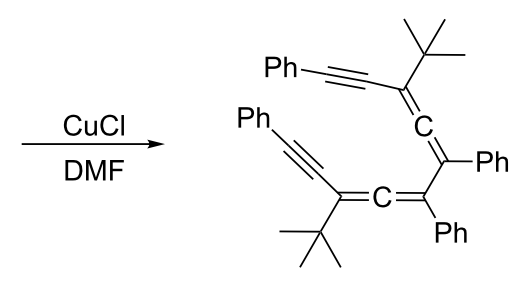

$122(11 \%)$<smiles>CC(C)(C)C(O)(C#CPc1ccccc1)c1ccccc1</smiles><smiles>CC(C)(C)C(=C\c1ccccc1)/C=C(/C#CC(c1ccccc1)C(C)(C)C)c1ccccc1</smiles>

$123(11 \%)$

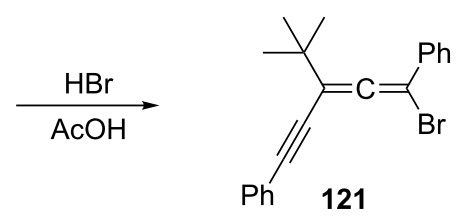

121

$124(54 \%)$

Scheme 32: The preparation of alkynyl-substituted bisallenes by a $\mathrm{C}_{3}$-dimerization protocol.<smiles>[R6][R16]=C[Na]</smiles> 
troscopic viewpoint as carefully as the parent system 1,2,4,5hexatetraene (2). Not only have its vibrational spectra been measured and interpreted $[92,93]$, but its most stable conformation has also been determined by electron diffraction in the vapor phase [94]. The results obtained by both of these physical methods are consistent with $C_{2 h}$-symmetry of the molecule, i.e. the transoid conformation is the most stable one. From its ${ }^{1} \mathrm{H}$ NMR spectrum [95], measured in a nematic solvent at room temperature, it has also been concluded that the hydrocarbon prefers a planar transoid structure with $C_{2 h}$-symmetry, the terminal $\mathrm{CH}_{2}$-groups being orthogonal to the plane passing through the other atoms. Furthermore, the photoelectron spectra of 2 (and several of its isomers) as well as of some simple alkyl derivatives have been studied [96,97], as has been that of the tetramethyl derivative $\mathbf{2 4}[98,99]$.

Although 2 is a liquid at room temperature and its structure in the solid state has not been determined so far, many X-ray structural investigations of single crystals of derivatives of $\mathbf{2}$ have been carried out. We will present those performed on acyclic bisallenes in this Section and will return to the structures of various cyclic bisallenes in Section 4.2. As revealed by a structure search in the Cambridge Crystallographic Data files [100] about 42 bisallenes have presently been studied by X-ray structural analyses. Out of these, 21 have been carried out on acyclic bisallenes, including their $d, l$ and meso-forms. Table 1 summarizes the structures of these derivatives and gives the leading references, which the reader can consult for further information.

\subsection{The chemical behavior of conjugated bisallenes 1.4.1 Pericyclic reactions}

That 2 and its derivatives may be of interest for the study of pericyclic reactions becomes obvious when one looks at the electronic structure of these molecules. First of all there is a high concentration of $\pi$-electron density in a relatively small space: eight $\pi$-electrons distributed over six carbon atoms. This is always a good prerequisite for (enhanced) chemical reactivity, especially when one is dealing with energy-rich substrates as in the case of the biallenyls. Furthermore, the particular arrangement of double bonds in 2 leads to two types of $\pi$-electron (sub)systems: "internally" 2 possesses a conjugated diene system and "externally" it displays two cumulated ones. For the case of cycloadditions (see below) this should allow for $[2+4]$ (Diels-Alder) and [2+2]cycloadditions (additions to allenes, ketenes, etc.). As we shall see later, both modes of cycloadditions have actually been observed.

Our discussion on pericyclic reactions of $\mathbf{2}$ will start with thermal processes, i.e. isomerizations that are induced by heating this compound and its derivatives under various conditions. As already pointed out previously, this is also one of the oldest reactions known for conjugated bisallenes. After the first isolation of 2 it was quickly demonstrated that on heating (either in solution or in the gas phase) it cyclizes to 3,4-bismethylenecyclobutene (130) in quantitative yield (Scheme 34) [6]. Up to the present day this electrocyclization remains the most thoroughly investigated reaction of the conjugated bisal-

Table 1: X-ray crystallographic studies on acyclic conjugated bisallenes of type 2 .

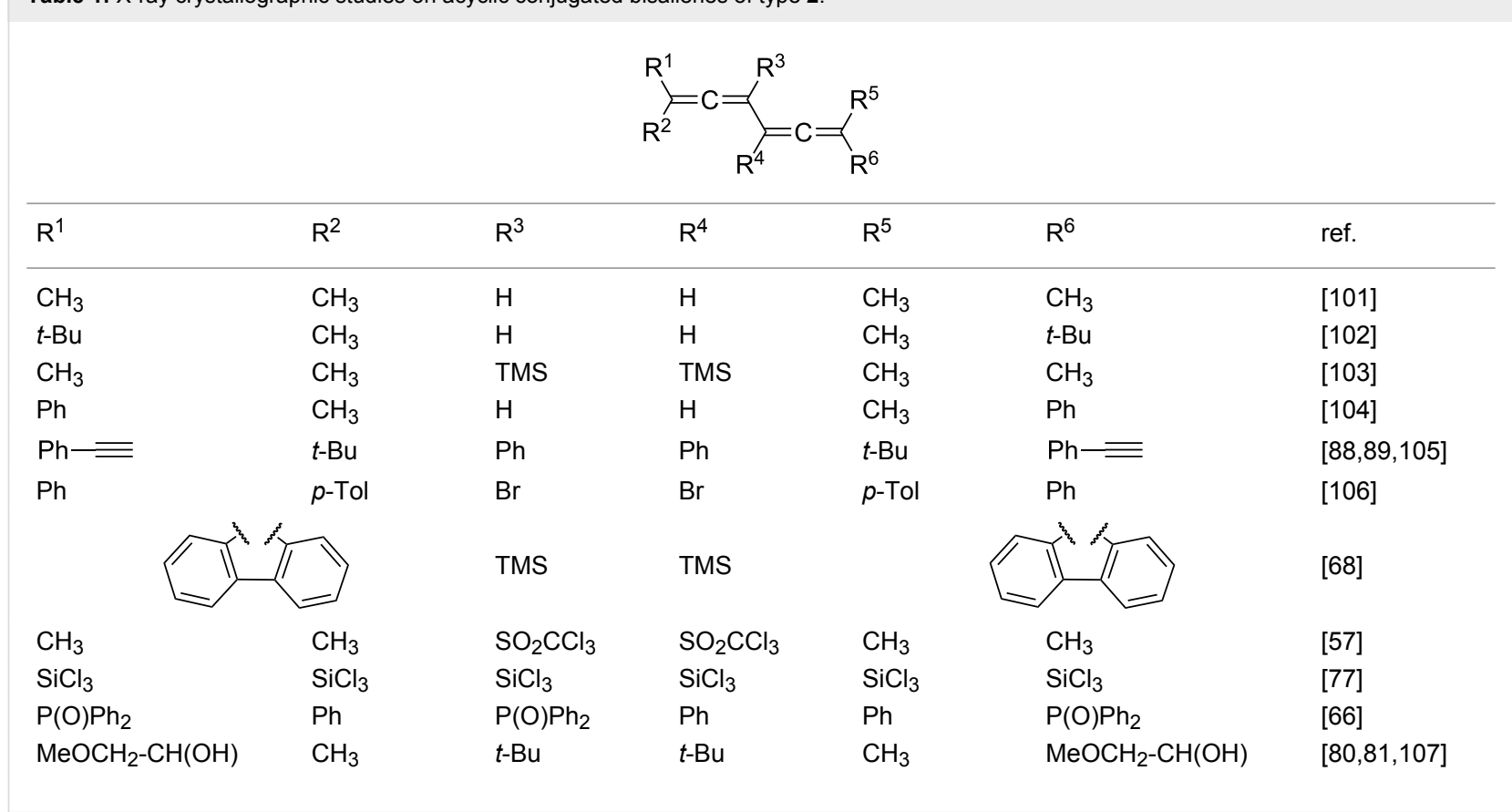


lenes. As a matter of fact, these studies also demonstrate that 2 is the most likely intermediate in the thermal interconversion of 1,5-hexadiyne (129) to 130, a Cope-type rearrangement that was discovered and discussed thoroughly by Huntsman and co-workers [108-111]. A shock-tube pyrolysis study of $\mathbf{2}$ in the temperature range of 540 to $1180 \mathrm{~K}$ at two nominal pressures ( 22 and $40 \mathrm{kbar}$ ) revealed that 2 plays an important role in the so-called propargyl mechanism, which is of importance in relation to the formation of aromatic compounds from nonaromatic precursors [112]. A radical cation Cope rearrangement of 1,5-hexadiyne (129) to the 1,2,4,5-hexatetraene radical cation was initiated by oxidizing bipropargyl radiolytically in a Freon matrix at $77 \mathrm{~K}$ [113]. The $\mathbf{1 2 9} \rightarrow \mathbf{2} \rightarrow \mathbf{1 3 0}$ sequence, and in particular its last step, has also been used several times for the preparation of functionalized bismethylenecyclobutenes, as demonstrated, for example, in Scheme 14.

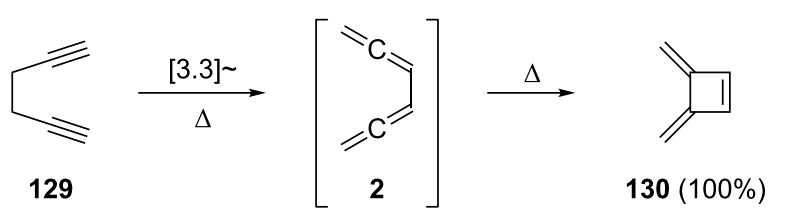

Scheme 34: The cyclization of 1,5-hexadiyne (129) to 3,4-bismethylenecyclobutene (130) via 1,2,4,5-hexatetraene (2) as a reaction intermediate.

If we look at the very early studies of Marvel and co-workers (see above, Introduction and Section 1.1), we can then rationalize the rearrangement of, e.g. 49 into 3 (Scheme 8, Section 1.1). The thermochemical parameters of the $\mathbf{2} \rightarrow \mathbf{1 3 0}$ cyclization have been determined and the activation parameters $\left(E_{\mathrm{a}}=\right.$ $29.3 \mathrm{kcal} / \mathrm{mol}, \log A=13.6$ ) [114,115] clearly speak for a concerted pericyclic process. This is, indeed, corroborated by studying the stereochemistry of the process, the work having been carried out by Huntsman [108,109], Pasto [29,115,116], Skattebøl [117] and others [118].

As shown in Scheme 35, the electrocyclization occurs in a strictly conrotatory manner and the stereoselectivity of the process depends on the steric size of the terminal substituents. For example, bisallene $\mathbf{1 3 1}$ has two distinct conrotatory modes for ring closure: counterclockwise and clockwise, providing the two bismethylenecyclobutenes $\mathbf{1 3 2}$ and 133, respectively. For derivative $\mathbf{1 3 4}$ the two conrotatory routes lead to $\mathbf{1 3 5}$ and $\mathbf{1 3 6}$ with a slight preference for the presumably sterically less hindered bismethylenecyclobutene 136. In 137 with its highly space-filling tert-butyl substituent this preference for the "outside" arrangement of the voluminous substituent is clearly more pronounced (vastly preferred formation of $\mathbf{1 3 9}$ over $\mathbf{1 3 8}$ ).

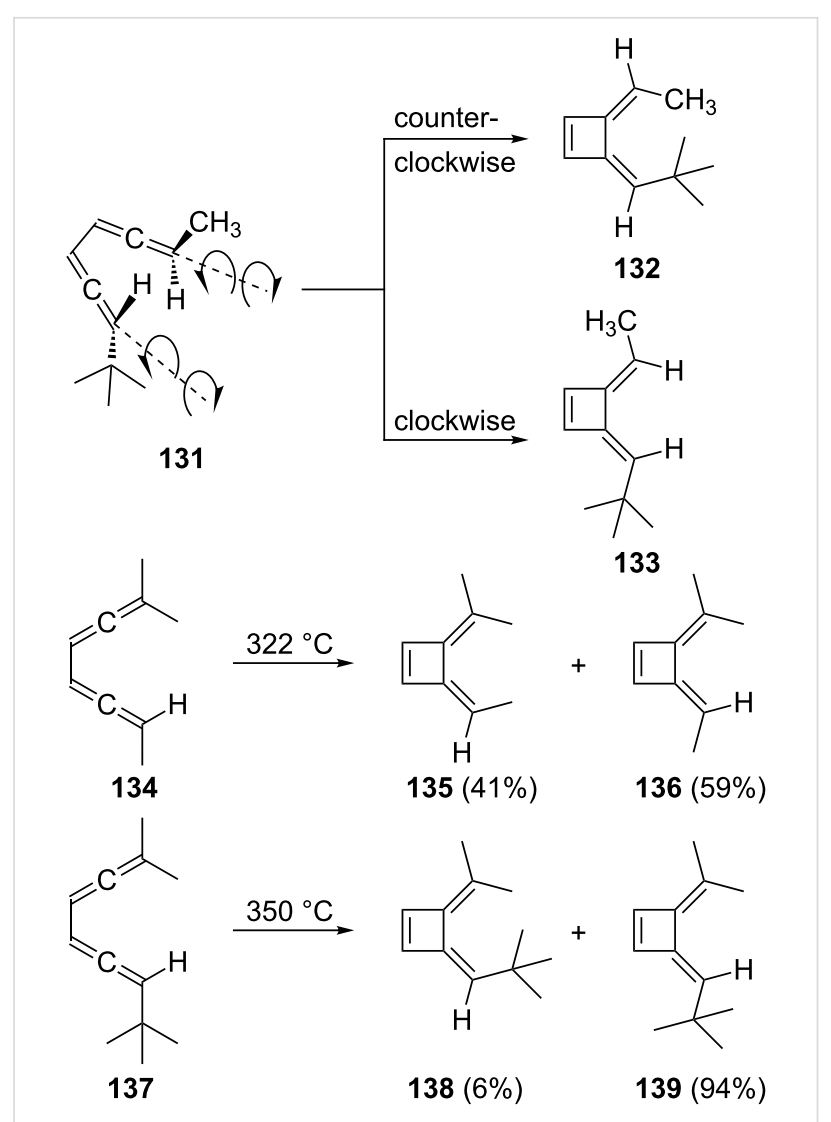

Scheme 35: Stereochemistry of the thermal cyclization of bisallenes to bismethylenecyclobutenes.

In a remarkable series of investigations Toda and co-workers have investigated the bisallene $\rightarrow$ bismethylenecyclobutene isomerization of the dialkynylated $d, l$ - and meso-bisallenes, 122 and $\mathbf{1 2 3}$ respectively, in the solid state (Scheme 36) [119]. These rearrangements occur in high yield and with high stereoselectivity.

When 122 was heated in benzene it isomerized to 145 in quantitative yield within $2 \mathrm{~h}$. A thorough analysis of the transformation, including its study in the solid state [105], revealed that it begins with a rotation around the central single bond, thus converting the transoid structure into the cisoid conformer $\mathbf{1 4 0 .}$ Conrotatory ring closure subsequently leads to the bismethylenecyclobutene 141. The alternative, which would provide isomer 142 with two endo-pointing tert-butyl groups, is avoided. In 141 the two phenethynyl moieties are so close that the hydrocarbon readily cyclizes to $\mathbf{1 4 5}$. The situation for mesocompound $\mathbf{1 2 3}$ is similar. After the initial conformational change to 143 ring closure provides the in,out-isomer $\mathbf{1 4 4}$, which cyclizes to 145 via the same intermediate 141 . The overall process is not only of interest because it involves transformations from single crystal to single crystal but also because of the benzocyclobutadiene derivatives $\mathbf{1 4 5}$, which are obtained 
<smiles>CC(C)(C)C(=C=C(C(=C=C(C(C)(C)C)C(C)(C)C)c1ccccc1)c1ccccc1)C#CPc1ccccc1</smiles>

122<smiles>CC(C)(C)C(=C=C(C(=C=C(C#Cc1ccccc1)C(C)(C)C)c1ccccc1)c1ccccc1)C#Cc1ccccc1</smiles>

123<smiles>CC(C)C1=C(C#Cc2ccccc2)C(=C(c2ccccc2)c2ccccc2)C(C)(C)C1</smiles><smiles>CC(C)(C)C(C#Cc1ccccc1)=C1C(=C(C#Cc2ccccc2)C(C)(C)C)C(c2ccccc2)=C1c1ccccc1</smiles>

141<smiles>CC(C)(C)C(C#Cc1ccccc1)=C1C(=C(C#Cc2ccccc2)C(C)(C)C)C(c2ccccc2)=C1c1ccccc1</smiles><smiles>CC(C)(C)C(C#Cc1ccccc1)=C1C(=C(C#Cc2ccccc2)C(C)(C)C)C(c2ccccc2)=C1c1ccccc1</smiles><smiles>CC(C)(C)c1c(-c2ccccc2)c2c(C(C)(C)C)c3c(-c4ccccc4)c-2c(-c2ccccc2)c(-c2ccccc2)c13</smiles>

145

Scheme 36: Bisallene $\rightarrow$ bismethylenecyclobutene ring closures in the solid state.

in unprecedented ease and display anomalies in their bond lengths $\left(\mathrm{C}_{\mathrm{sp}}{ }^{2}-\mathrm{C}_{\mathrm{sp}}{ }^{2}\right.$ bonds as long as $\left.1.540 \AA\right)$ [120]. For another route to benzocyclobutenes see Section 2.4, Scheme 71 .
A most interesting electrocyclization/dimerization process has been discovered by McGlinchey and co-workers for the disilylated hydrocarbon 86 (Scheme 37; see also Scheme 19, Section

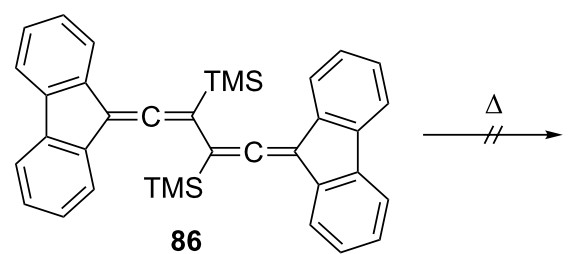
$110^{\circ} \mathrm{C} \downarrow$<smiles>CC(C)=C1c2ccccc2-c2ccccc21</smiles><smiles>CSC1=C(S)C(=C2c3ccccc3-c3ccccc32)C2=C(S1)C(C)=C2C</smiles>

146

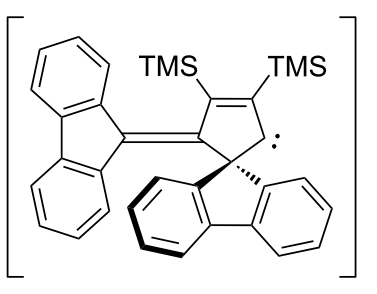

148

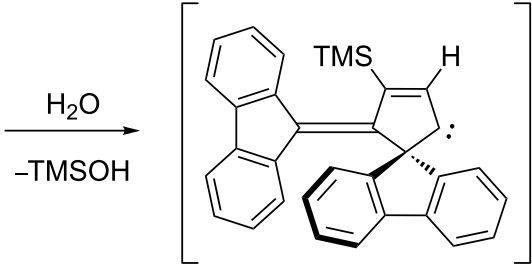

149

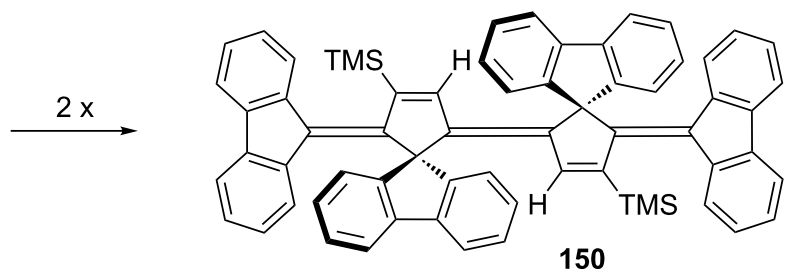

Scheme 37: A bisallene cyclization/dimerization reaction. 
1.2) [68]. Heating the highly hindered conjugated bisallene 86 in toluene does not lead to the expected bismethylenecyclobutene 146; instead the isomerization takes a completely different course. This time no monomeric but a dimeric product is formed, i.e. the unusually structured TMS-derivative 150. To rationalize this surprising result, the authors propose that after the conformational change of 86 to 147 , formation of the fivemembered carbene 148 occurs, which by loss of one TMSsubstituent is transformed into the carbene 149. And this ultimately dimerizes to $\mathbf{1 5 0}$. Derivative $\mathbf{1 5 0}$ can be desilylated to a $\mathrm{C}_{60} \mathrm{H}_{36}$ hydrocarbon, whose $\mathrm{C}_{36}$ backbone represents ca. $60 \%$ of the fullerene framework and which can be mapped onto $\mathrm{C}_{60}$ [121].

A pericyclic reaction of conjugated bisallenes which has also been studied extensively over the years involves the Diels-Alder addition with both double and triple bond dienophiles, as well as heteroatom containing dienophiles. This is demonstrated by several examples in Scheme 38. Most classes of double-bond dienophiles react in the expected fashion and provide the Diels-Alder adducts in good yields, for example, 152, 155, 158 and $\mathbf{1 6 0}$. Whenever there are two $\alpha$-hydrogen atoms in the adduct aromatization can be accomplished by base treatment (e.g. formation of 153 and 156).
Incorporation of substituents into the double bond of the dienophile slows down the addition process or prevents it completely. Unusual dienophiles such as cyclobutadiene or various 1,2-disubstituted cyclobutenes do not add to 2 [122].

To investigate the stereochemistry of addition both $1,2,4,5$ heptatetraene (161) [122] and the erythro-bisallene 163 [116] were subjected to Diels-Alder additions (Scheme 39) with maleic anhydride (151) and $N$-phenylmaleimide (154), respectively. In both additions the dienophiles attack the bisallene (in $s$-cis conformation) from the less hindered face to provide the diastereomers 162 and 164, respectively, in an exclusive fashion.

The azetidinones $\mathbf{1 1 3}$ described above (see Scheme 29) have also been employed in various cycloaddition experiments with double-bond dienophiles, 165; the resulting Diels-Alder adducts 166 were subsequently aromatized to 167 by DBUtreatment (Scheme 40) [85].

Whereas the reaction of 1,1,6,6-tetramethyl-1,2,4,5-hexatetraene (24) with $N$-sulfinylaniline (168) gave the $[2+4]$ cycloadduct 169 as the only isolable product (Scheme 41), the reaction of $\mathbf{2 4}$ with either dichloro $(\mathbf{1 7 0}: \mathrm{R}=\mathrm{Cl})$ or<smiles>C=C=CC=C</smiles>

2<smiles>[R]C1=C([R])C(=O)OC1=O</smiles>

151

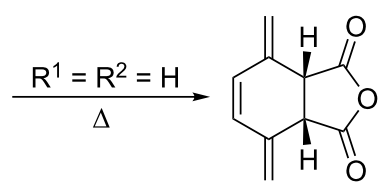

$152(80 \%)$

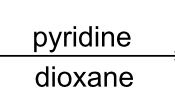

$20^{\circ} \mathrm{C}, 10 \mathrm{~min}$

.<smiles>C=C1C=CC(=C)[C@]2(C)C(=O)N(c3ccccc3)C(=O)[C@H]12</smiles>

$155(60 \%)$

pyridine<smiles>Cc1ccc(C)c2c1C(=O)OC2=O</smiles>

$153(83 \%)$<smiles>O=C1C=CC(=O)N1Pc1ccccc1</smiles><smiles>CCCC</smiles><smiles>Cc1ccc(C)c2c1C(=O)N(c1ccccc1)C2=O</smiles>

$156(70 \%)$<smiles>C=C1C=CC(=C)C2(C)C(=O)C(Cl)=C(Cl)C(=O)C1(C)C2=O</smiles><smiles>C=C(C=CC)N1C(=C)C=CC(=C)N1C(=O)OCC</smiles> 
<smiles>O=C1C=CC(=O)O1</smiles>

151<smiles>CC#CC=CCC</smiles>

161<smiles>C=C1C=C/C(=C/C)[C@H]2C(=O)OC(=O)[C@H]12</smiles>

162<smiles>O=C1C=CC(=O)N1[PH3+]</smiles>

154<smiles>CC=CC=CC=CC</smiles>

163<smiles>C/C=C1\C=C/C(=C\C(C)(C)C)[C@H]2C(=O)N(c3ccccc3)C(=O)[C@H]12</smiles>

Scheme 39: The stereochemistry of the $[2+4]$ cycloaddition to conjugated bisallenes.

diphenylketene (170, $\mathrm{R}$ = phenyl) furnished largely the $[2+2]$ cycloadducts $\mathbf{1 7 1}$. Only traces of the Diels-Alder adduct $\mathbf{1 7 2}$ are observed in this case. This example seems to be the only one in which the $[2+4]$ and $[2+2]$ cycloaddition mode compete [123]. Occasionally highly alkylated bisallenes such as $\mathbf{2 4}$ also provide ene-addition products.

If the double-bond dienophile is replaced by an activated triplebond dienophile 173, another course of events is observed (Scheme 42) [21,124-126]. In this case the Diels-Alder step generates a $p$-xylylene intermediate 174 (a $p$-quinodimethane) first, which subsequently stabilizes itself by dimerization; the tetrasubstituted [2.2]paracyclophanes $\mathbf{1 7 5}$ are produced in acceptable yields (max. 50\%). This route constitutes the preparatively most satisfactory way to obtain highly functionalized [2.2]paracyclophanes. Unactivated triple bonds do not undergo cycloaddition (no reaction with tolane, 2-butyne, cyclooctyne, etc.).

Switching to asymmetric triple-bond dienophiles, such as propiolic aldehyde (176), leads to a monosubstituted $p$-xylylene intermediate $\mathbf{1 7 7}$, which has four different options to dimerize. These provide the isomeric bisformyl [2.2]paracyclophanes $\mathbf{1 7 8}$ to $\mathbf{1 8 1}$ in about equal ratio and in a total yield of up to $45 \%$ [127]. The apparently disadvantageous production of a mixture of isomers is not a real handicap of this route since these adducts differ in their physical properties (polarity, solubility) strongly enough to allow easy separation/purification by chromatography and/or recrystallization. The different isomers 178-181 are interesting starting materials in cyclophane chemistry in their own right. Other asymmetrically substituted triplebond dienophiles that have been used like 176 include cyanoacetylene and methyl propiolate.

A third group of pericyclic reactions of conjugated bisallenes that has been studied thoroughly concerns various cheletropic cycloadditions. The earliest studies in this area involve the addition of sulfur dioxide to different alkyl and aryl-substituted bisallenes (Scheme 43) [117]. The reaction yields the 1:1 adducts and from the stereochemistry of these products it must be concluded that the sulfur dioxide approaches the bisallenes from the least-hindered side and that the new bonds are formed under disrotation as illustrated in formula 183 [128].<smiles>[R]C1C(=O)NN1C(=C=C)C=C=C</smiles>

113

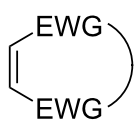

165<smiles>C1CCCCC1</smiles>$$
\text { O }
$$

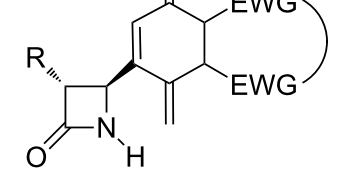

166

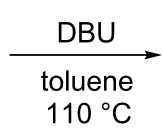

$110^{\circ} \mathrm{C}$<smiles>[R]C1C(=O)NC1c1cc(C)c(C#N)c(C)c1C#N</smiles>

167

Scheme 40: Preparation of azetidinone derivatives from conjugated bisallenes.

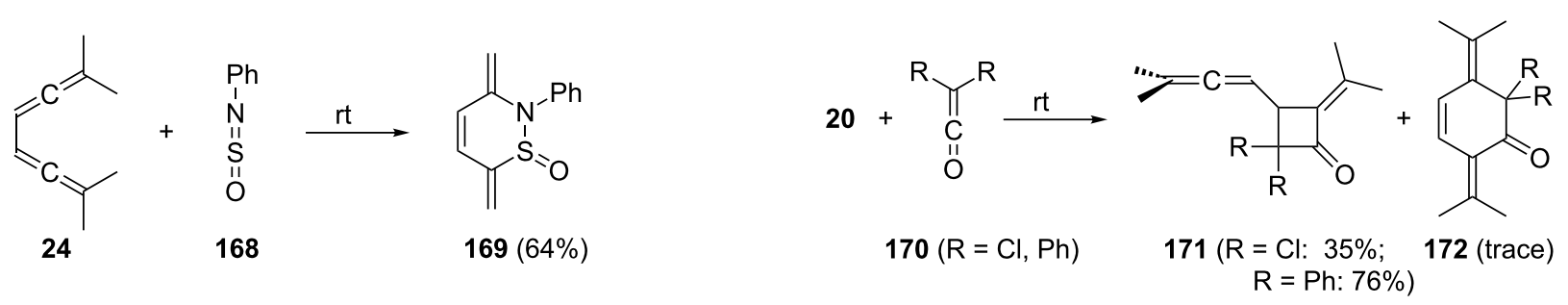




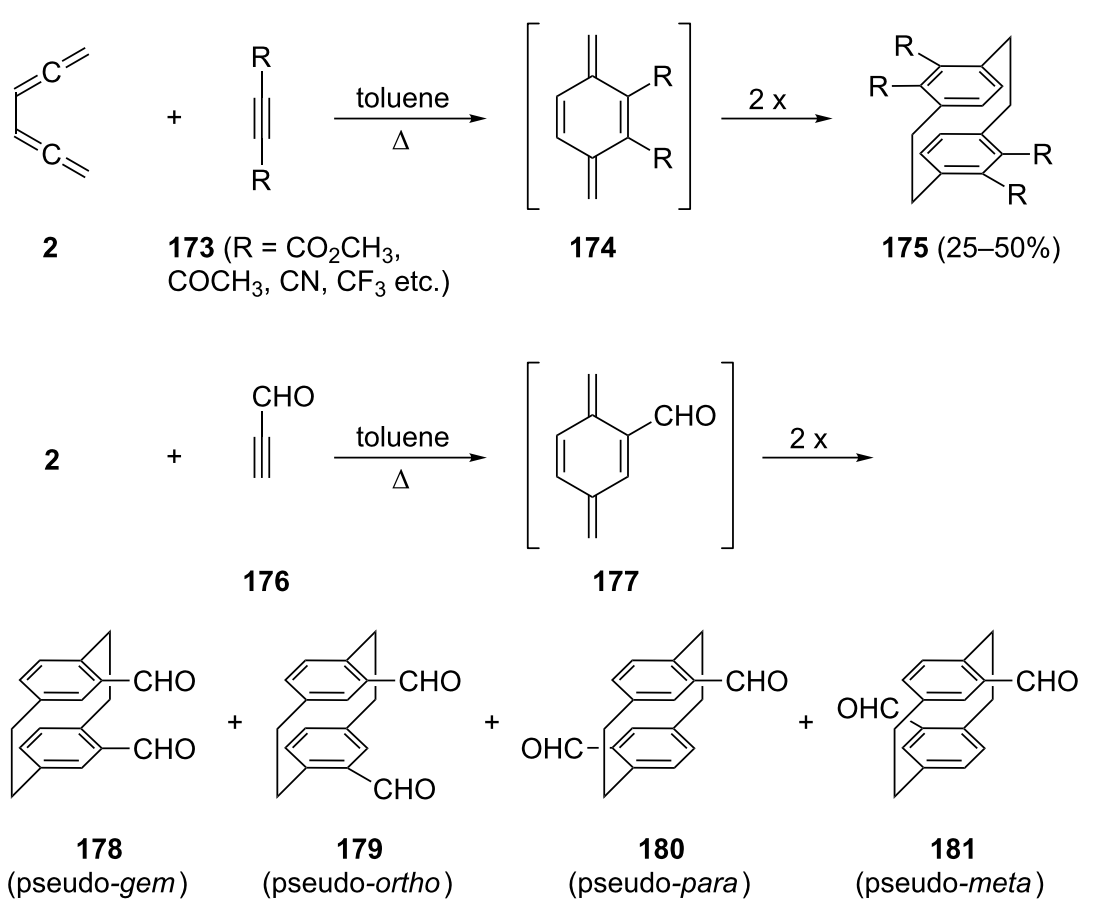

Scheme 42: Addition of triple-bond dienophiles to conjugated bisallenes.

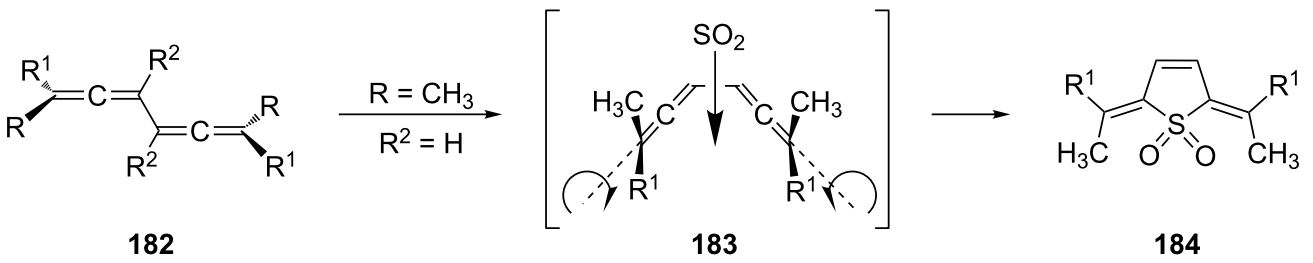

Scheme 43: Sulfur dioxide addition to conjugated bisallenes.

As far as the stereochemistry is concerned, several other addends behave similarly, as shown, e.g. by the germylene $\mathbf{1 8 5}$, which provides the adduct 187 with 186 (Scheme 44). Likewise, the meso-compound $\mathbf{1 8 8}$ leads to $\mathbf{1 8 9}$ and $\mathbf{1 9 0}$. There is no crossover between the two sets of reactions underlining their concerted nature. That $\mathbf{1 8 9}$ is the main product in the cycloaddition can be rationalized by the attack of $\mathbf{1 8 5}$ from the least shielded side of $\mathbf{1 8 8}$, i.e. from the side of the methyl substituents. In summary, the germylene behaves as a singlet species in an orbital-symmetry-controlled cycloaddition (cheletropic, disrotatory cycloaddition) [129]. In an earlier study the Neumann group had already shown that stannylenes such as $\mathrm{Me}_{2} \mathrm{Si}(\text { tert }-\mathrm{BuN})_{2} \mathrm{Sn}$ or $\left[\left(\mathrm{Me}_{3} \mathrm{Si}\right)_{2} \mathrm{CH}\right]_{2} \mathrm{Sn}$ behave similarly towards the bisallenes $\mathbf{1 8 6}$ and $\mathbf{1 8 8}$ [130-132].

The tetramethylhexatetraene $\mathbf{2 4}$ was also reacted with various phosphinidenes generated in situ from various stable precursors.
For example, the tungsten-complexed pentacarbonyl complex $\mathrm{PhP}=\mathrm{W}(\mathrm{CO})_{5}$, generated by heating 191 (retro-Diels-Alder reaction), provided the unexpected 3,4-disubstituted phosphole 192 (Scheme 45) [133]. Analogously, the phospholene complex 194 resulted from heating 24 and 193 together [134].

Finally, as shown by Lüttke and Heinrich, the cyclopropanation of the parent system 2 with diazomethane $/ \mathrm{Cu}_{2} \mathrm{Cl}_{2}$ provided a mixture of twelve cyclopropanation products in very good yield (ca. 90\%), from the mono adduct 195 to the dispiro compound 196, i.e. the 4:1 adduct (Scheme 46) [135]. The chemical behavior of these novel strained hydrocarbons has not been reported yet.

In comparison to thermal (pericyclic) processes of conjugated bisallenes, very little is known about their photochemical behavior regardless of the mechanism of these processes. In fact 

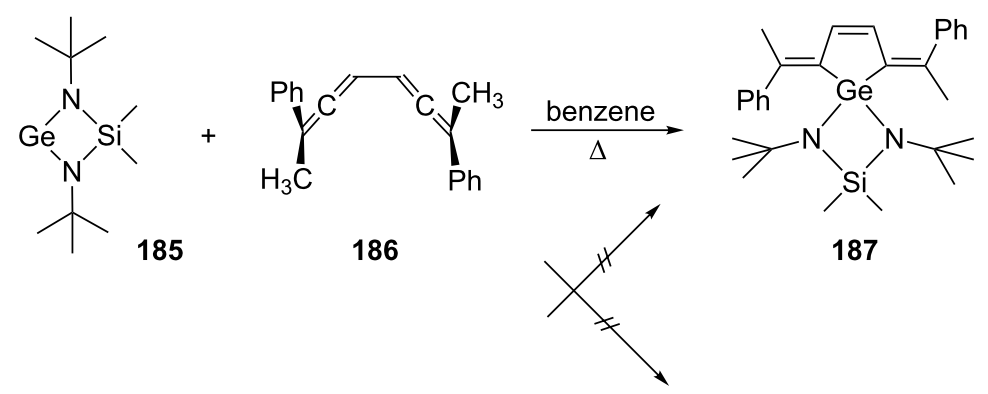

187

185

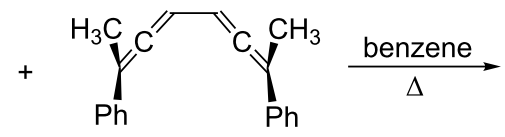

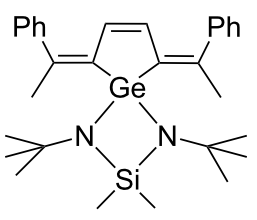

189

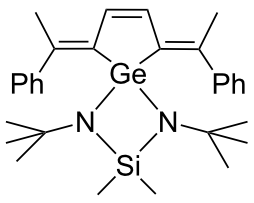

$190(189: 190=8: 1)$

Scheme 44: The addition of a germylene to a conjugated bisallene.<smiles>CC(C)=CC=C(C)C</smiles>

24<smiles>COC(=O)C1=C(C(=O)OC)C2CC(C1C(=O)OC)P2c1ccccc1</smiles>

191<smiles></smiles>

192

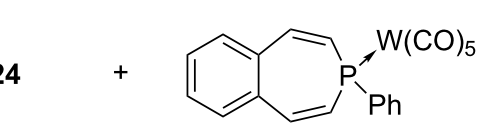

193

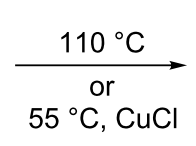

$\stackrel{65^{\circ} \mathrm{C}}{\longrightarrow}$

194

Scheme 45: Trapping of conjugated bisallenes with phosphinidenes.

$$
=\mathrm{C}=\underset{\mathrm{C}=\frac{\mathrm{CH}_{2} \mathrm{~N}_{2}}{\mathrm{Cu}_{2} \mathrm{Cl}_{2}} \mathrm{~V}}{=\mathrm{C}=+\ldots+}
$$

Scheme 46: The cyclopropanantion of 1,2,4,5-hexatetraene (2).

we could only find two references describing photoreactions involving bisallenes. Thus, irradiation of the epoxide 197 in benzene- $d_{6}$ (NMR experiment) yielded a plethora of photoproducts, among them two photodimers to which structure 198 was assigned by spectroscopic measurements (Scheme 47) [136]. In the second experiment, the parent hydrocarbon 2 was irradiated in the presence of vinylacetylene (199) and a triplet sensitizer (benzophenone). Besides the photodimers of 199, the [2+2] photoadduct 200 was isolated in low yield [137].<smiles>CC(=O)C#CC1(C(C)C)OC1(C)C</smiles>

197<smiles>CC(=O)C(=CC(=CC(C(C)C)C(C)(C)O)C(C)(C)O)C(C)=O</smiles>

198

$2+\underset{\sim}{\stackrel{h v, \text { ether }}{\text { benzophenone }}}$

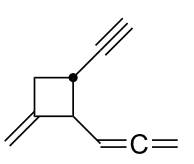

199

Scheme 47: Photochemical reactions involving conjugated bisallenes. 


\subsubsection{Ionic reactions}

Regardless of what types of reactions are summarized under this title, whether they involve carbocations or carbanions, or "only" polarized intermediates, ionic reactions involving $\mathbf{2}$ and its derivatives have only been studied to a limited extent so far And this situation does not change very much if we include the chemical behavior of cyclic bisallenes as discussed in Section 4.2 .

Probably the earliest ionic reaction involving a conjugated bisallene concerns Kuhn's hydrocarbon 4 [9]. On treatment with base it isomerizes in good yield to the vinylbutatriene derivative 201. Later it was shown that the parent system 2 isomerizes under comparable conditions into a mixture of 202-204 (Scheme 48) [39].
The addition of one equiv of protic acids $(\mathrm{HCl}, \mathrm{HBr})$ or iodine to 1,1,6,6-tetramethyl-1,2,4,5-hexatetraene (24), provided the diastereomeric 2,5-dihalogenated trienes $\mathbf{2 0 5}$ and 206 as shown in Scheme 49. Addition of excess bromine to $\mathbf{2 4}$ furnished the tetrabromide 207, which on hydrolysis $\left(\mathrm{H}_{2} \mathrm{O}, \mathrm{Al}_{2} \mathrm{O}_{3}\right)$ provided the dibromo diol 208 [138].

A careful study of the oxidation of several conjugated bisallenes with different oxidants has been carried out by Pasto and co-workers [139]. As summarized in Scheme 50, the tetramethylbisallene $\mathbf{2 4}$ yields two products with meta-chloroperbenzoic acid ( $m$-CPBA): the alkylidenecyclopentenone 209, and the "open" oxidation product $\mathbf{2 1 0}$ in whose formation the carboxylate anion resulting from the oxidation reagent is obviously involved. With dimethyldioxirane (DMDO) two products are produced again: $\mathbf{2 0 9}$ and the bisoxygenated product $\mathbf{2 1 1}$ for<smiles>C(=C=C=C(c1ccccc1)c1ccccc1)=C=Cc1ccccc1</smiles>

4<smiles>C=C=CC=C=C</smiles>

2<smiles>COC(=O)OCC(=O)O</smiles><smiles>C(=C=Cc1ccccc1)=C=C(c1ccccc1)c1ccccc1</smiles>

$201(70 \%)$

Scheme 48: Base-catalyzed isomerizations of conjugated bisallenes.

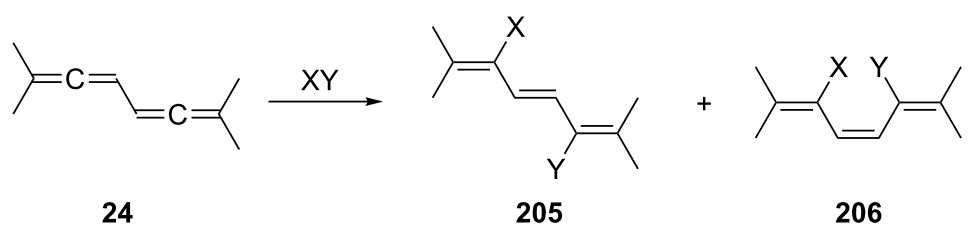

\begin{tabular}{cccc} 
X & Y & 205/206-ratio & yield, \% \\
\hline H & $\mathrm{Cl}$ & $10: 1$ & 45 \\
$\mathrm{H}$ & $\mathrm{Br}$ & $20: 1$ & 53 \\
$\mathrm{I}$ & $\mathrm{I}$ & only 205 & 82
\end{tabular}

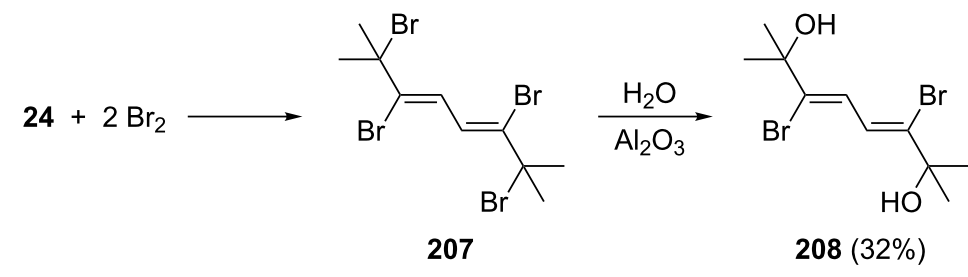

Scheme 49: lonic additions to a conjugated bisallene. 


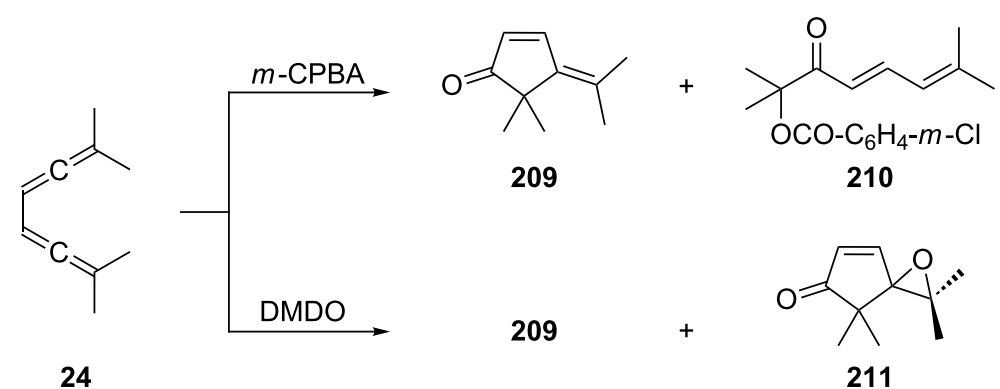

Scheme 50: Oxidation reactions of a conjugated bisallene.

which 209 appears to be the most reasonable precursor. The oxidation of $\mathbf{2 4}$ with magnesium monoperoxyphthalate provides $\mathbf{2 0 9}$ in 32\% isolated yield [138]. Looking at the structures of starting material and product $\mathbf{2 0 9}$ the process resembles the Nazarov cyclization of divinyl ketones to cyclopentenones [140].

Applying the mechanism discussed in this latter context to $\mathbf{2 4}$, it is reasonable to propose the formation of an allene-epoxide first [141], which can exist either in a transoid, 212, or cisoid conformation, 213 (Scheme 51). Whereas the former could open up to the "stretched" zwitterion 214, which by carboxylate interception would lead to $\mathbf{2 1 0}$, the more "closed" allenoxide $\mathbf{2 1 3}$ could isomerize to $\mathbf{2 1 5}$, which on ring closure would furnish 209 and 211, respectively. A 1:1 mixture of erythro and threo8,8-dimethyl-2,3,5,6-nonatetraene behaves similarly, with the exception that this hydrocarbon is already oxidized by standing in air; the tetramethyl derivative $\mathbf{2 4}$ is stable towards air [139].

\subsubsection{Metal-induced reactions}

Although metal-initiated reactions (usually cyclizations) have become important ring-forming processes for the "higher" and heteroorganic bisallenes (see Sections 2.3 and 6.2), they are also known for conjugated bisallenes. We also would like to include metalation reactions in this chapter, namely the reactions we have already briefly referred to above (see Sections 1.1 and 1.2). Another example encountered earlier is the transformation recorded in Scheme 25, where silver and/or gold salts were employed to induce the cyclization of functionalized conjugated bisallenes.

One of the first examples of a metal-catalyzed isomerization involves 1,2,4,5-hexatetraene (2) itself. When a suspension of 2 and $\mathrm{CuCl}$ in ether is stirred at room temperature the bisallene cyclizes to 3,4-bismethylenecyclobutene (130) in quantitative yield (Scheme 52) [142]. Many substituted bisallenes behave similarly and isomerize to the corresponding derivatives of $\mathbf{1 3 0}$ in a conrotatory manner [29].

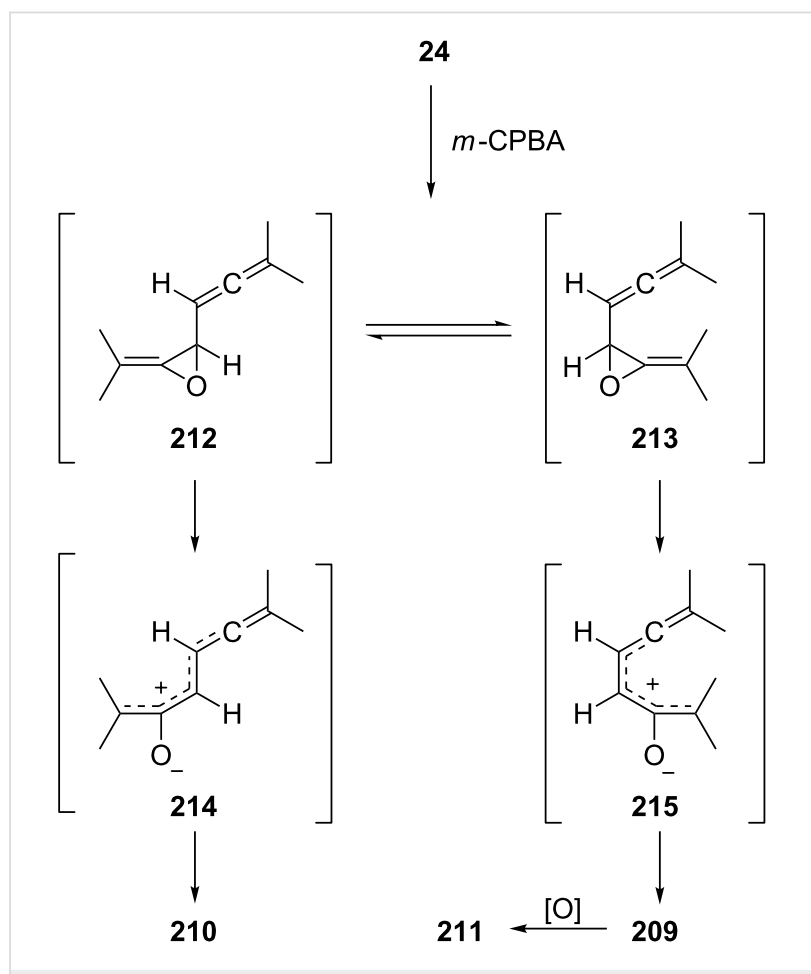

Scheme 51: The mechanism of oxidation of the bisallene 24 .

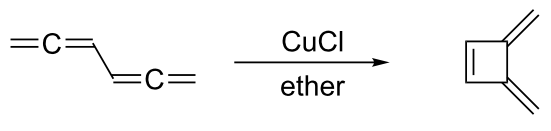

2

$130(100 \%)$

Scheme 52: CuCl-catalyzed cyclization of 1,2,4,5-hexatetraene (2).

An interesting cyclization/functionalization of acyclic bisallenes was observed by Eaton and Rollman (Scheme 53) [102]. The cyclopentenone derivatives $\mathbf{2 1 6 / 2 1 8}$ are obtained in very good yields and the reaction takes place in a short time, as demonstrated for the model bisallene 24 in Scheme 53. In a later, more comprehensive study the scope and stereochemistry of the process were investigated thoroughly [24]. As can be 
seen in Scheme 53 the reaction takes place with high stereoselectivity, transferring the relative stereochemistry of the substrate bisallene to the product dialkylidenecyclopentenone. This outcome speaks for the $\eta^{4}$-coordination of a s-cis-diallene conformation and rules out a mechanism whereby facial selectivity is determined by $\eta^{2}$-coordination.

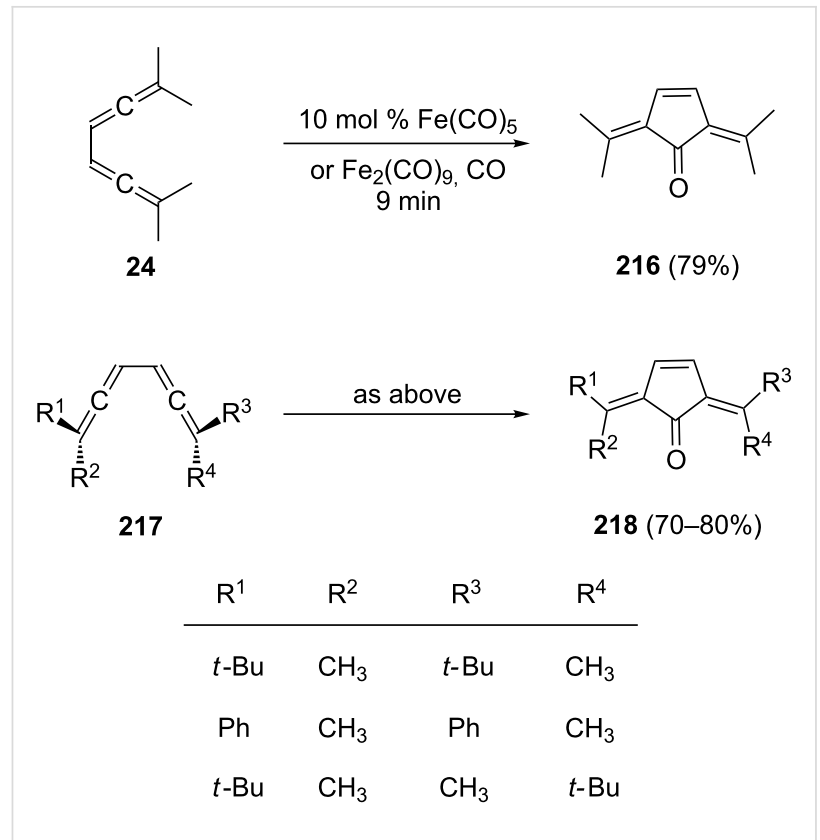

Scheme 53: The conversion of conjugated bisallenes into cyclopentenones.

The behavior of $\mathbf{2 4}$ towards nickel catalysts has been studied by Pasto and Huang (Scheme 54) [143]. In the presence of $\mathrm{Ni}\left(\mathrm{PPh}_{3}\right)_{3} 24$ trimerizes to the unusual ten-membered polyene 219. When the reaction is carried out with $\mathrm{Ni}(\mathrm{COD})_{2}(\mathrm{COD}=$ 1,5-cyclooctadiene) both the direct cycloisomerization product of the bisallene, hydrocarbon $\mathbf{2 2 0}$, and the symmetrical dimer 221 are isolated. In the presence of the mixed ligand complex $\mathrm{Ni}(\mathrm{COD})\left(\mathrm{PPh}_{3}\right)$ a mixture of $\mathbf{2 2 0}$ and $\mathbf{2 2 1}$ is produced.

The metalations of bisacetylenic and bisallenic hydrocarbons already referred to (Section 1.2) [74-76] can probably also be exploited to introduce other substituents than TMS. Thus the quenching of the corresponding carbanions with, e.g., carbon dioxide to produce carboxylic acid derivatives of conjugated bisallenes could be tried $[83,138,144]$.

\section{Acyclic nonconjugated bisallenes}

\subsection{1,2,5,6-Heptatetraene and its derivatives}

The simplest conceivable acyclic, nonconjugated bisallenic hydrocarbon is 1,2,5,6-heptatetraene (229), a compound that has evidently escaped synthesis on a preparatively useful scale so far (Scheme 55). A reasonable route to prepare this hydro-

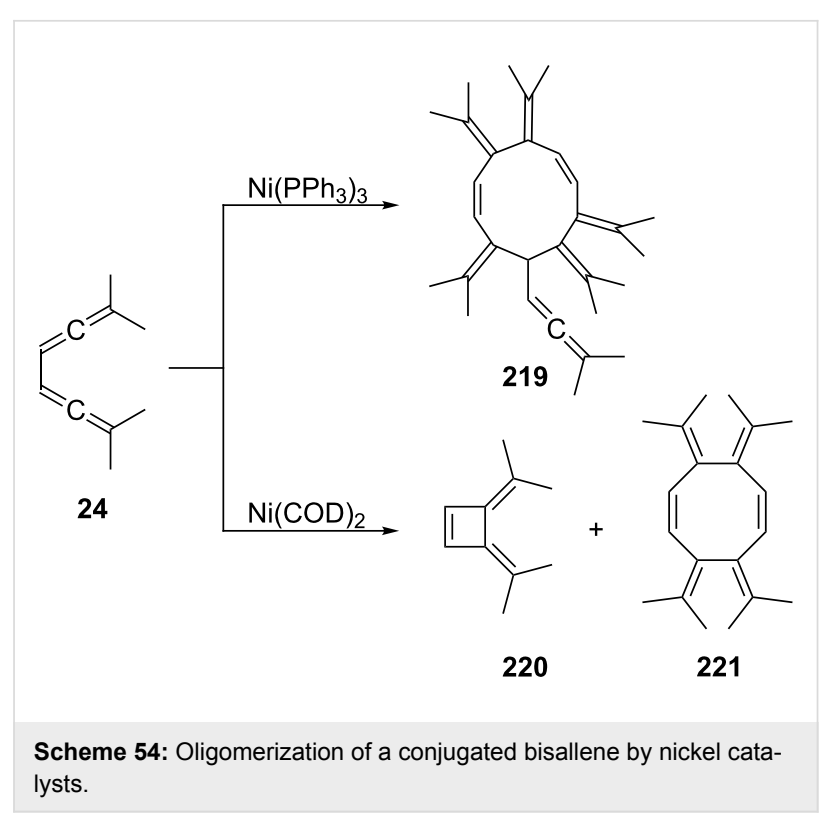

carbon started from the dibromocarbene adduct of 1,4-pentadiene, the tetrabromide $222[25,145]$. However, when this was subjected to the DMS-allene synthesis (see Section 1.1), a rather complex mixture of hydrocarbons resulted in poor yield

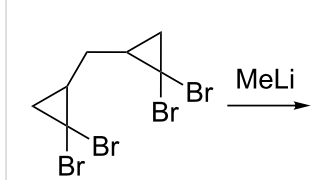

222<smiles>[R6][C@H]1C=CC(=C=C)C1</smiles>

228
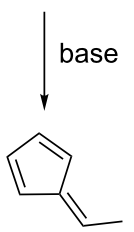

231<smiles>C=CCCC(C)C</smiles>

223

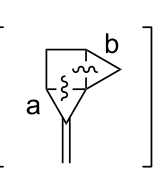

224
Scheme 55: Generation of 1,2,5,6-heptatetraene (229) as a reaction intermediate. 


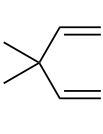

234

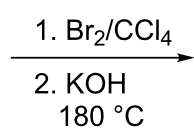

$180^{\circ} \mathrm{C}$

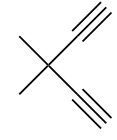

235

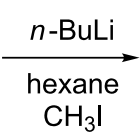

$\mathrm{CH}_{3} \mathrm{I}$

Scheme 56: The preparation of a stable derivative of 1,2,5,6-heptatetraene.

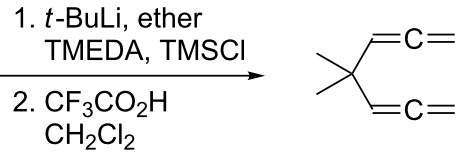

237 (ca. 10\%), consisting of the two fulvenes $\mathbf{2 3 1}$ and $\mathbf{2 3 3}$ as well as 1,2,4,5-heptatetraene (232). A mechanism accounting for the formation of these unsaturated hydrocarbons is summarized in Scheme 55 and involves the generation of the intermediates $\mathbf{2 2 3}$ to 230. According to this rationalization the immediate precursor of $\mathbf{2 3 2}$ is the desired but not isolated nonconjugated bisallene 229 .

That it is, indeed, the activated methylene moiety of $\mathbf{2 2 9}$ which plays a decisive role in its stability was demonstrated by the preparation of its 4,4-dimethyl derivative 237 by Roth and co-workers (Scheme 56) [146,147]. Starting from the geminally dimethylated 1,4-pentadiene $\mathbf{2 3 4}$, these workers converted this diene by conventional steps via $\mathbf{2 3 5}$ into the 2,5-heptadiyne derivative $\mathbf{2 3 6}$, which was subsequently subjected to a double prototropic rearrangement to yield the bisallene 237 .

Another way to remove the reactive $\alpha$-hydrogen atoms in $\mathbf{2 2 9}$ consists of replacing them by functional groups. A case where this approach was successful has been reported by Ried and Marhold [148], who have prepared the dichloroketone 239 from $\mathbf{2 3 8}$ by $\mathrm{HCl}$ treatment. If $\mathbf{2 3 9}$ itself is exposed to hydrochloric acid for longer times it cyclizes to the cross-conjugated ketone 240 (Scheme 57).<smiles>COC(C#CC(=O)C#CC(c1ccccc1)(c1ccccc1)c1ccccc1)(c1ccccc1)c1ccccc1</smiles>

Scheme 57: A bisallene with a carbonyl group as a spacer element.

\subsection{1,2,6,7-Octatetraene and its derivatives}

The next higher homologue of $\mathbf{2 2 9}$, the $\mathrm{C}_{8}$-hydrocarbon $\mathbf{2 4 2}$ was first prepared by Skattebøl in the founding days of modern allene chemistry applying the DMS-protocol to 1,5-hexadiene
(241, Scheme 58) [149]. The hydrocarbons 242 and 243 are produced in a total yield of $71 \%$ in $2.5: 1$ ratio, the tricyclic hydrocarbon $\mathbf{2 4 3}$ evidently generated by trapping of a cyclopropylidene intermediate by an already formed allene unit. Later work by the same authors showed that this approach can be extended to substituted [150] and also to functionalized [151] 1,2,6,7-octatetraenes. A direct conversion of $\alpha, \omega$-diynes into their chain-extended $\alpha, \omega$-diallenes by a Simmons-Smith reaction has also been described [152]; it yields mixtures of linear hydrocarbons, though, requiring chromatography for separation/purification.

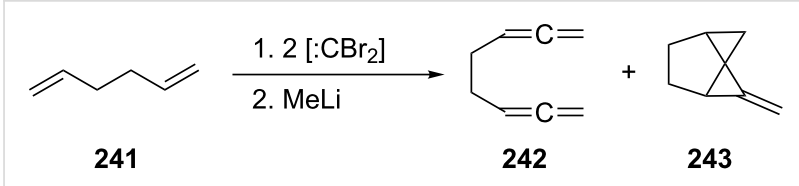

Scheme 58: The first preparation of 1,2,6,7-octatetraene (242).

Not surprisingly, several approaches to the $\mathrm{C}_{8}$-hydrocarbon system 242 by a $\left(\mathrm{C}_{4}+\mathrm{C}_{4}\right)$-coupling protocol have been reported. These involve either enyne substrates or appropriately functionalized $\mathrm{C}_{4}$ building blocks. For example, alkyl and arylsubstituted enynes $\mathbf{2 4 4}$ on lithium treatment in THF followed by a water quench provide a mixture of the dimers 245 and 246 (Scheme 59) [153]. The dimer mixture is produced in acceptable yield (50-87\%), and the bisallene is normally the dominant component. With trimethylsilyl chloride as the trapping agent, silyl-substituted bisallenes such as $\mathbf{2 4 7}$ are obtained.

The second approach referred to above, may be illustrated by the dimerization (Grignard coupling) of different (homo)allenic bromides $\mathbf{2 4 8}$ in the presence of magnesium (Scheme 60) [154]. Although the yields are poor (10-20\%), the bisallenes 251 always constitute the main dimer of the reaction. Note that one of the dimers, 249, has the structure of a [4]dendralene [48].

Substituted bisallenes of type $\mathbf{2 4 2}$ have also been obtained by applying Crabbé's cuprate method to dipropargylic diacetates (Scheme 61) [155], a protocol that has also been employed for the preparation of various cyclic bisallenes (see Section 4.2.2). The mixture of the diastereomers 253 (meso) and $254(d, l)$ is 


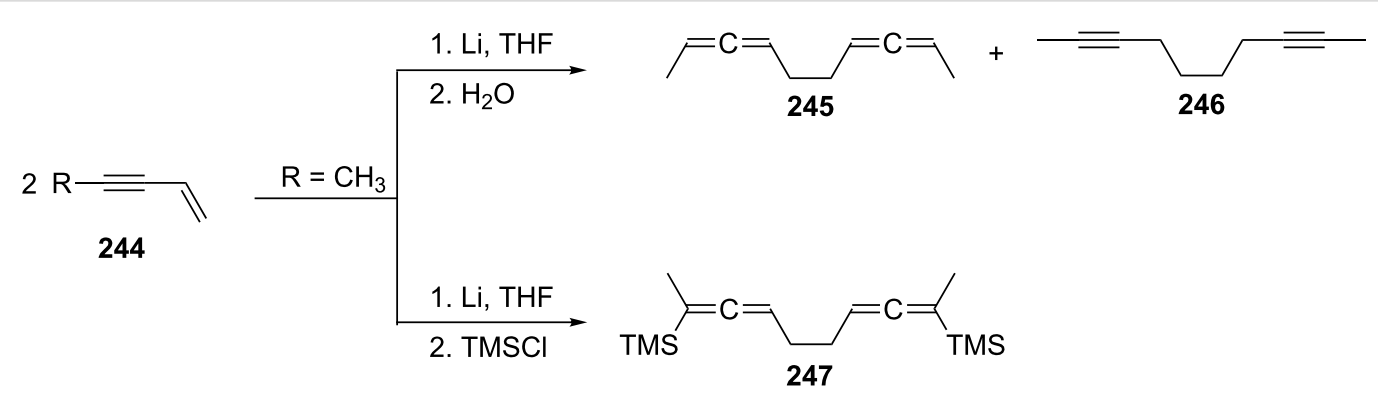

Scheme 59: Preparation of 1,2,6,7-octatetraenes by $\left(C_{4}+C_{4}\right)$-coupling of enynes.<smiles>[R]C=C=C([R])CBr</smiles>

248<smiles>[R]C=C(C(=C)C)C([R])C([R])=C</smiles>

249<smiles>[R]C=C=C([R])CC([R])=C</smiles>

250<smiles>[R]C=C=C([R])CCC([R])=C[R]</smiles>

251

Scheme 60: Preparation of 1,2,6,7-octatetraenes by $\left(\mathrm{C}_{4}+\mathrm{C}_{4}\right)$-coupling of homoallenyl bromides.

produced in excellent yield (91\%). Functionalized 1,2,6,7octatetraenes 257 (ethers) are isolated in moderate yields (50-60\%) when the protected bipropargyl derivative 255 was treated with different acetals $\mathbf{2 5 6}$ under the influence of titanium(IV) chloride. The resulting bisallenes $\mathbf{2 5 7}$ are useful substrates for the preparation of benzocyclobutenes, as will be discussed in Section 2.4 (Scheme 71) [156].

We conclude this section with the preparation of two remarkable, highly substituted bisallenes of type $\mathbf{2 4 2}$ (Scheme 62). In the first transformation it was shown that the 1,5-hexadiyne derivative $\mathbf{2 5 8}$ on acid treatment undergoes an unusual rearrangement to the bisallene 259. It is likely that the ring strain of the two cyclopropane rings contributes significantly to the driving force of the process [157]. The second reaction involves the diketone 260, which on treatment with the $\mathrm{ZnCl}$ salt of propargyl chloride yields largely the $d, l$-diastereomer
261. On reduction of $\mathbf{2 6 1}$ with Red-Al the bisallene $\mathbf{2 6 2}$ is obtained, which is a useful substrate for Pauson-Khand ringformation reactions (see below, Section 2.4, Scheme 71) [158].

\subsection{Higher acyclic $\alpha, \omega$-bisallenes}

Since the direct interaction of the two allene moieties is the most important preparative question related to acyclic bisallenes, it is not surprising that the number of publications dealing with the "higher" bisallenes, i.e. those where the spacer between the allene groups becomes longer and longer, is rather small and decreases with the length of the spacer. These more extended derivatives often just display the chemical behavior of two separate allene units. Still, surprisingly, a number of bisallenes has been described in which the number of unsubstituted (methylene) or substituted carbon atoms separating the allene units is three, four or even higher and reactions still take place between the allenic termini.<smiles>C#CC(C)(C#C)OC(C)(C#C)CCC(C)=O</smiles>

252<smiles>[R]C([R])[R6]</smiles><smiles>CC=C=C(C)CCC(C)=C=CC</smiles>

253

254

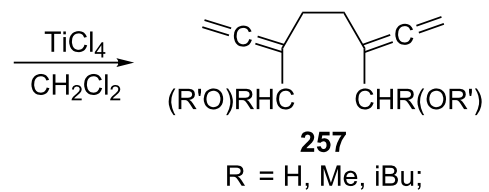

$\mathrm{R}^{\prime}=\mathrm{CH}_{3}, \mathrm{Et}$ 


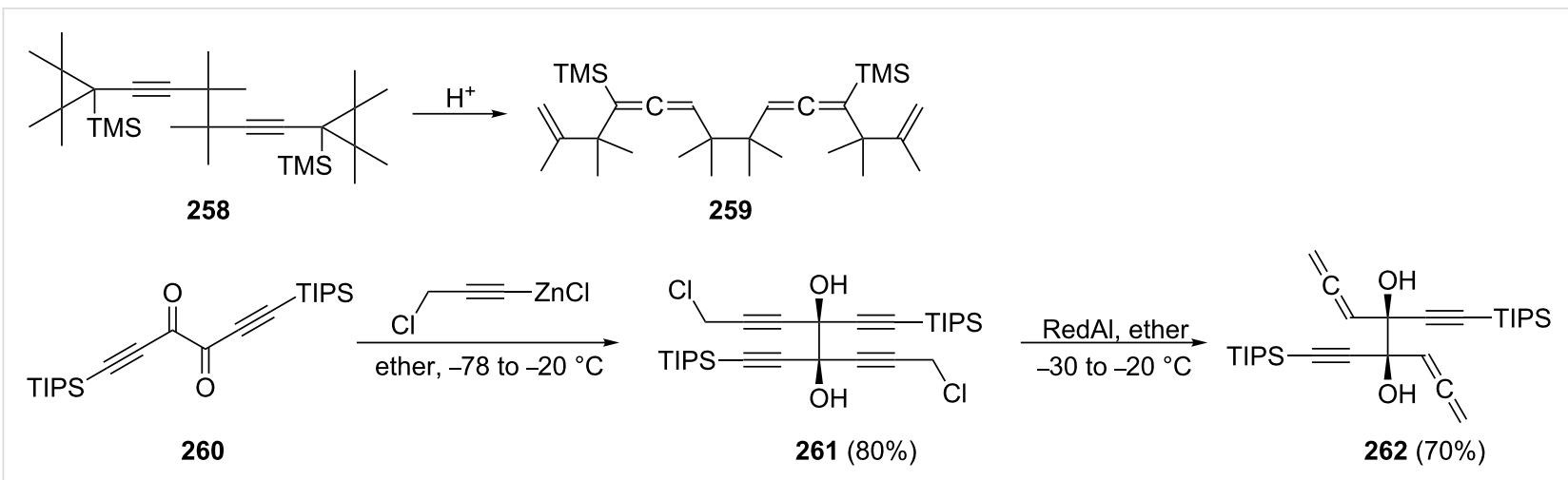

Scheme 62: Preparation of two highly functionalized 1,2,6,7-octatetraenes.

In fact many of the parent hydrocarbon systems are known, a case in point being $\mathbf{2 6 5}$ and $\mathbf{2 6 6}$, which have been synthesized from the corresponding bis(dibromocyclopropane) derivatives 263 and 264 in very good yield by the DMS-route (Scheme 63) [149]. Actually the transformation is more complex, as was shown in a later investigation, at least at low temperatures. As demonstrated by Brinker [159] under these conditions besides, e.g. 1,2,7,8-nonatetraene (265), which is still the main product of the debromination/rearrangement, other hydrocarbons are formed, among them 267, a formal 1,5-CH-insertion product, and $\mathbf{2 6 8}$, which results by way of a 1,7-cycloelimination process (Scheme 63). The 1,2,8,9-decatetraene (266) has also been obtained by dimerization of 1-bromo-3,4-pentadiene with magnesium in THF (Grignard coupling) in 30\% yield [160].

Several alkyl derivatives of $\mathbf{2 6 5}$ and $\mathbf{2 6 6}$ have been prepared and although their importance in preparative organic chemistry is far lower than that of the functionalized derivatives described below, it is useful to have access to them for spectral comparison. For example, coupling of the bismetallic reagent 269 with propargyl bromide provides the $n$-butyl derivative of $\mathbf{2 6 5}$, the branched hydrocarbon 270, in 48\% yield (Scheme 64) [161]. The Ni-catalyzed cross-coupling reaction of the diacetylenic

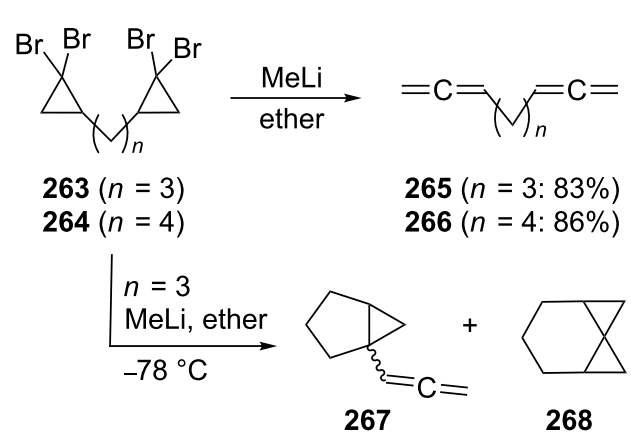

Scheme 63: Preparation of several higher $\alpha, \omega$-bisallenes.

dithioketal 271 with Grignard reagents such as methylmagnesium iodide furnished the hexamethyl derivative of bisallene 266, hydrocarbon 272 [162].

For the preparation of functionalized derivatives of $\mathbf{2 6 5}$ and 266, as well as those in which their saturated methylene groups have partially been replaced by heteroatoms $(\mathrm{O}, \mathrm{N}$, see Section 6 below) or carry functional groups, a substantial number of methods exist. In an early study by Miginiac and Barbot the ethers $\mathbf{2 7 4}$ were prepared by reacting orthoesters at low

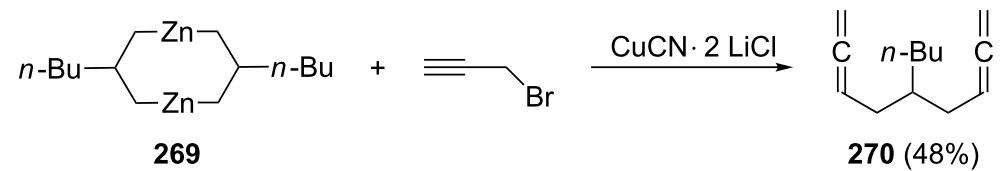

$270(48 \%)$

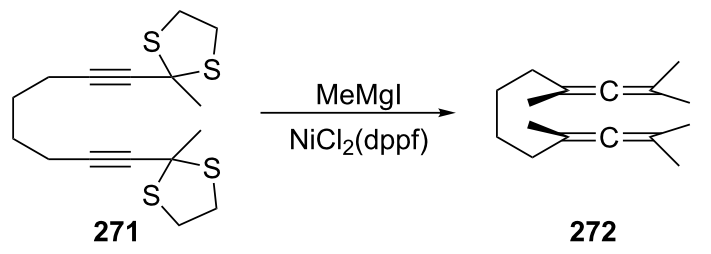


temperatures with homoallenic bromides 273 (Scheme 65) [163]. Various cyanoesters or malononitriles, 276, have been added to conjugated enynes $\mathbf{2 7 5}$ in the presence of a Pd-catalyst to generate the bisallenic nitriles 277 in fair yields [164]<smiles>[R]C=C=C([R])CBr</smiles>

273

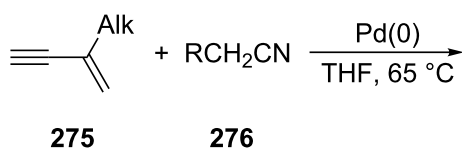<smiles>[R]C=C=C([R])CC(CC([R])=C=C[R])OCC</smiles>

$274\left(\mathrm{R}=\mathrm{H}, \mathrm{R}^{\prime}=\mathrm{CH}_{3}: 70 \%\right.$ $\left.\mathrm{R}=\mathrm{CH}_{3}, \mathrm{R}^{\prime}=\mathrm{H}: 55 \%\right)$<smiles>[R]C=C=C([AlH2])CC([R])(C#N)CC([AlH2])=C[R]</smiles>
$277\left(\mathrm{R}=\mathrm{CN}, \mathrm{CO}_{2} \mathrm{Me}\right.$; 15-60\%)
Scheme 65: The preparation of functionalized 1,2,7,8-nonatetraene derivatives.

Similar yields of the diallenic diesters $\mathbf{2 7 9}$ were obtained in the coupling of esters of $\alpha$-allenic alcohols (acetates and in particular phosphates such as 278) with the anions of $\beta$-diesters, $\beta$-ketoesters and $\alpha$-phenylsulfonyl esters in the presence of catalytic amounts of palladium $(0)$ complexes such as $\mathrm{Pd}\left(\mathrm{PPh}_{3}\right)_{4}$ (Scheme 66) [165,166]. The diester 279 is also obtained when unsubstituted malonate $\mathbf{2 8 0}$ is treated with excess 2-chloro-1,3butadiene (281, chloroprene) and 2.5 equiv of sodium methoxide in the presence of Pd/DPEphos [167]. Another Pd-mediated coupling experiment, leading to a derivative of 266 involves the dibromide $\mathbf{2 8 2}$ and the aromatic haloester 283; the bisallene derivative $\mathbf{2 8 4}$ was obtained in $68 \%$ yield [168].
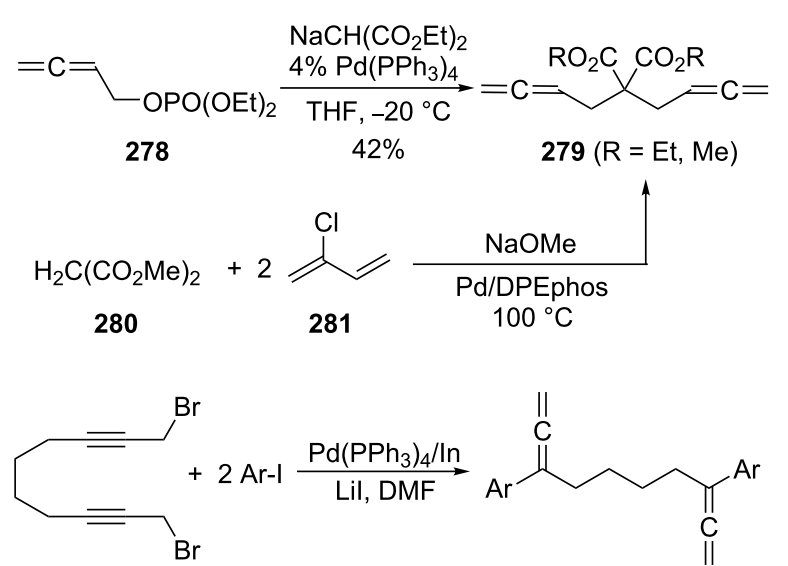

282

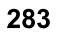

284

$$
\mathrm{Ar}=4-\mathrm{EtO}_{2} \mathrm{C}-\mathrm{C}_{6} \mathrm{H}_{4}
$$

Scheme 66: Preparation of functionalized $\alpha, \omega$-bisallenes.
Extended $\alpha, \omega$-bisallenes are often prepared from the corresponding $\alpha, \omega$-dienes by the DMS-route (see Section 1.1 above), but a direct homologation of an $\alpha, \omega$-diyne has also been reported. Thus, 1,2,9,10-undecatetraene (288) was prepared by Crabbé and co-workers from 1,8-nonadiyne (285) by a route involving the cuprous bromide-catalyzed homologation of the diacetylene with formaldehyde and diisopropyl amine in dioxane under reflux (Scheme 67) [169]. A mechanism has been proposed for this reaction involving a $\mathrm{CuBr}$-catalyzed Mannich reaction step followed by the pericyclic fragmentation of the initially produced intermediate $\mathbf{2 8 6}$ to the bisallene $\mathbf{2 8 8}$ and the imine 287.

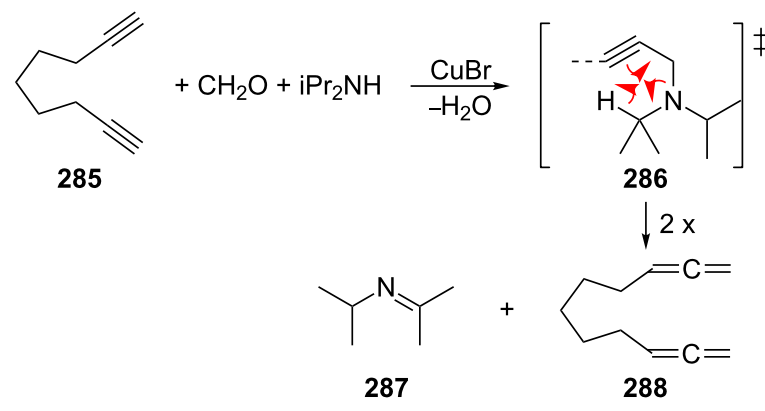

Scheme 67: The preparation of an $\alpha, \omega$-bisallene by direct homologation of an $\alpha, \omega$-bisalkyne.

As far as the still more elongated $\alpha, \omega$-bisallenes are concerned, it is surprising to what extent intramolecular reactions are observed once the spacer element is rigidified by, e.g., aromatic subunits. The corresponding examples will be discussed in greater detail below (see Section 4.1).

\subsection{The chemical behavior of nonconjugated, acyclic $\alpha, \omega$-bisallenes}

The structurally simplest of these hydrocarbons is $1,2,5,6$ heptatetraene (229), and its base-induced isomerization to 1,2,4,5-heptatetraene (232) has already been referred to in Scheme 55.

A thorough study of the thermal behavior of its 4,4-dimethyl derivative 237 was carried out by Roth, Maier and co-workers. As illustrated in Scheme 68, on heating, the dimethyl-substituted, and hence protected, hydrocarbon $\mathbf{2 3 7}$ first cyclizes to the diradical 289, an intermediate that can also be produced from other precursors [146]. In the absence of trapping agents, 2,2dimethyl-4,5-dimethylene-1,3-cyclopentanediyl (289) dimerizes to two dimers, i.e. 290 and 291, whereas interception with oxygen leads to 292, with $N$-phenylmaleimide (NPM) to adducts 293 and 294, and with sulfur dioxide to sulfolene 295. As we shall see below, structurally similar compounds are formed when the higher homologues of $\mathbf{2 2 9} / \mathbf{2 3 7}$ are pyrolyzed. 


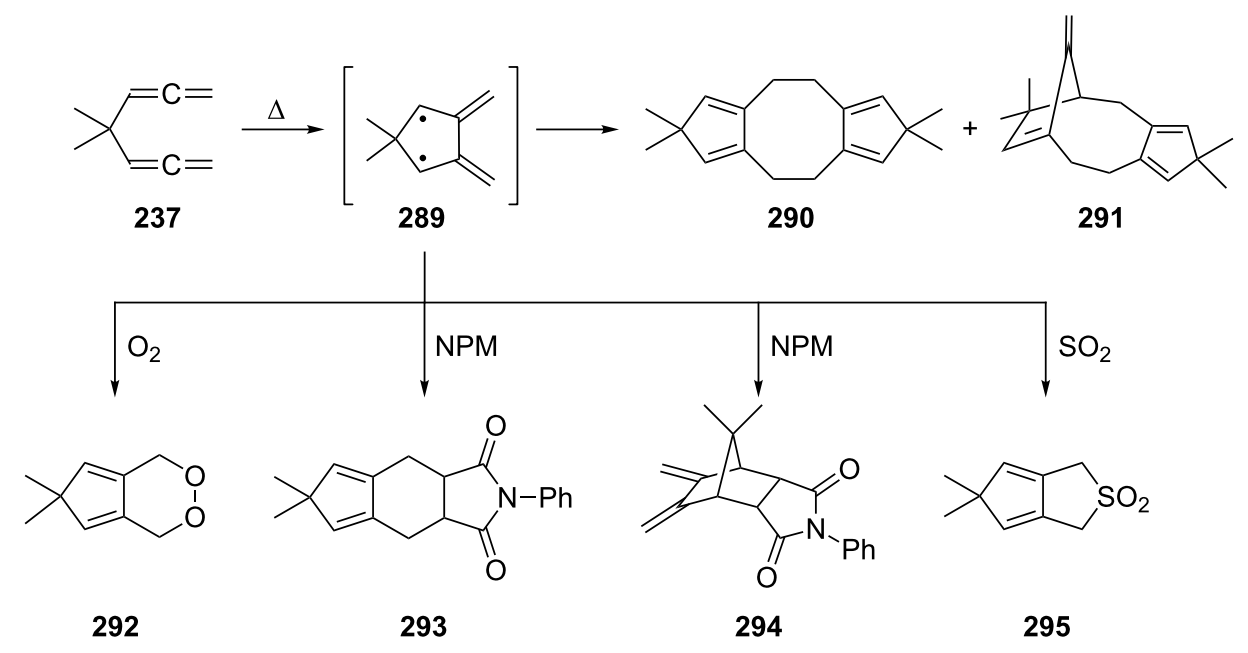

Scheme 68: The gas-phase pyrolysis of 4,4-dimethyl-1,2,5,6-heptatetraene (237).

The thermal cyclization of 1,2,6,7-octatetraene (242) was first studied by Skattebøl and Solomon (Scheme 69) [170]. On being heated in the gas phase at $310{ }^{\circ} \mathrm{C}$ hydrocarbon 242 rearranges quantitatively into 3,4-dimethylene-1,5-hexadiene (296). What formally may be regarded as an allenic variant of the classical Cope-rearrangement of 1,5-hexadiene (a subsystem of 242) is actually considerably more complex, as was shown by Roth [171-173], Grimme [174] and others. In fact not only 296 is generated under pyrolysis conditions, but also the $\mathrm{C}_{8} \mathrm{H}_{10}$ isomer bicyclo[4.2.0]octa-1,5-diene (298), and it was shown that the two hydrocarbons are in thermal equilibrium. As above, the diradical intermediate $\mathbf{2 9 7}$ may be trapped by added gases such as oxygen or sulfur dioxide to provide the 1:1 adducts 299-303.

The meso- and $d, l-2,3,7,8$-decatetraenes behave similarly and suggest the formation of diradicals of type $\mathbf{2 9 7}$ in these cases also [150]. A computational study (quantum chemical CI calculations with the semiempirical MO method SINDO1) on the $\mathrm{C}_{8} \mathrm{H}_{10}$ network involving the above hydrocarbons and intermediates has been carried out by Jug and Iffert [175].

The cyclopropanation $\left(\mathrm{CH}_{2} \mathrm{~N}_{2}, \mathrm{CuCl}\right)$ of $\mathbf{2 4 2}$, performed by de Meijere and Kaufmann [176], gave all possible mono- to tetraadducts. As in the case of 2 (see Section 1.4.1, Scheme 46) Scheme 70 only shows a selection of these nine products, 304-306; to separate and identify these rather similar cyclopropanes constitutes an impressive experimental feat. Again, it would be of interest to know, e.g. the thermal behavior of these hydrocarbons.

As far as derivatives of $\mathbf{2 4 2}$ carrying functional groups are concerned, we mention the already referred to bisallenic diethers 257, which, under pyrolysis conditions, cyclize in good

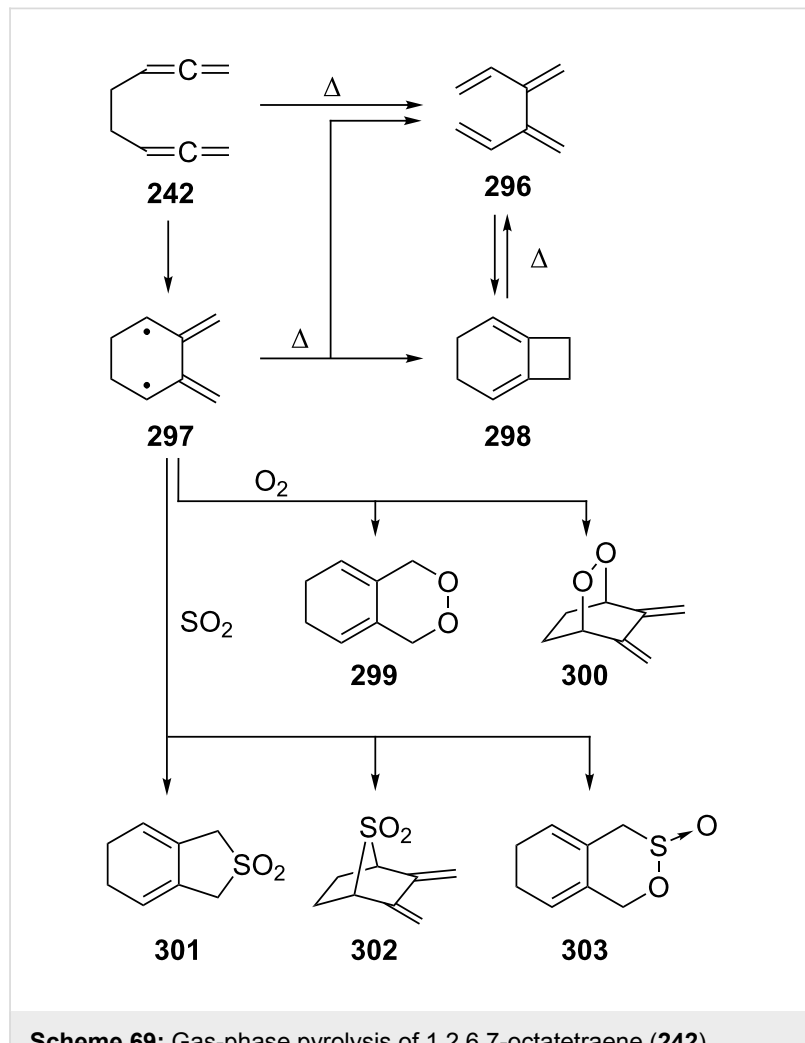

Scheme 69: Gas-phase pyrolysis of 1,2,6,7-octatetraene (242).

yields (55-80\%) to substituted benzocyclobutenes 309 involving the intermediates $\mathbf{3 0 7}$ and $\mathbf{3 0 8}$, as shown in Scheme 71 [156]. A second transformation involves the remarkable Pauson-Khand reaction of the highly functionalized 1,2,6,7-octatetraene derivative $\mathbf{3 1 0}$, which was already encountered in the form of the diol $\mathbf{2 6 2}$ in Scheme 62; with molybdenum hexacarbonyl it cyclizes to the dicyclopenta[a,e]pentalene derivative 311 [158]. 


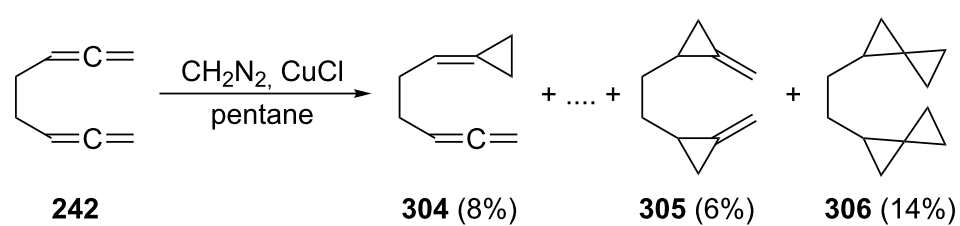

Scheme 70: The cyclopropanation of 1,2,6,7-octatetraene (242).

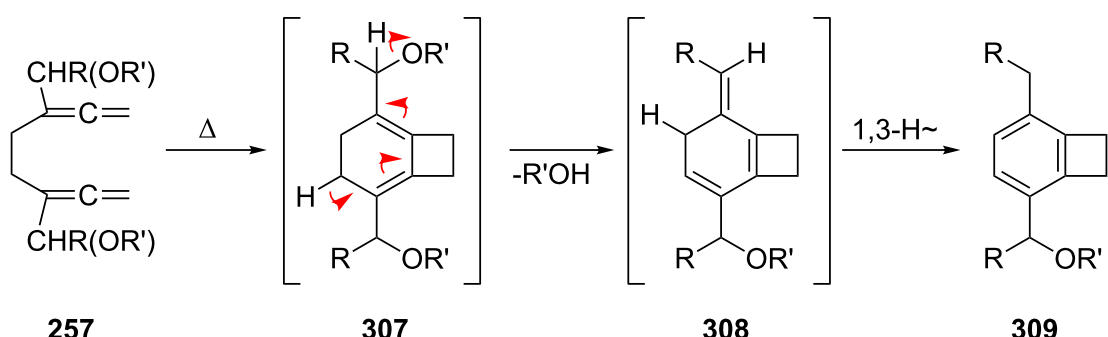

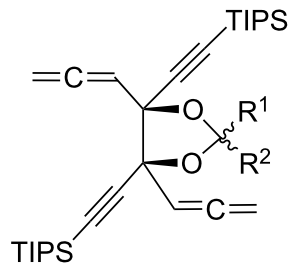

310
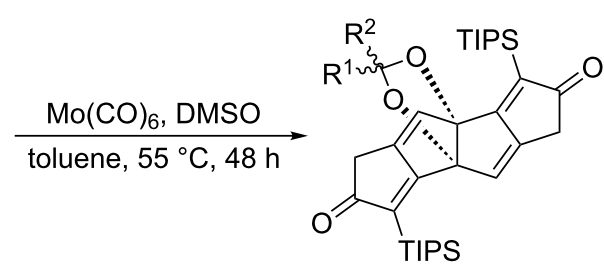

$311\left(65-70 \% ; \mathrm{R}^{1}=\mathrm{H}\right.$, $\mathrm{R}^{2}=p$-anisyl)

Scheme 71: Intramolecular cyclization of 1,2,6,7-octatetraene derivatives.

The next higher homologues of $\mathbf{2 4 2}, 1,2,7,8$-nonatetraene (265, see Scheme 63) and 1,2,8,9-decatetraene (266), were also subjected to gas-phase pyrolysis at 390 and $300{ }^{\circ} \mathrm{C}$, respectively, by Skattebøl and Solomon (Scheme 72) [170]. The former hydrocarbon cyclizes in an intramolecular [2+2] cycloaddition to 312 , i.e. it undergoes the reaction that is so typical (intermolecularly) for simple monoallenes; whereas the latter does not react, possibly as a consequence of the distance between the allene units being too large. The yield of the $\mathbf{2 6 5} \rightarrow \mathbf{3 1 2}$ transformation is poor $(32 \%)$ and the not fully characterized product mixture does not allow the proposal of a mechanism for this thermocyclization. When the temperature for $\mathbf{2 6 6}$ is increased to $440{ }^{\circ} \mathrm{C}$ a product mixture of unknown composition is obtained in low yield.

Recent years have witnessed numerous reports on the use of functionalized derivatives of $\mathbf{2 6 5}$ and $\mathbf{2 6 6}$ in preparative organic chemistry. In principle any position of these bisallenes can carry a functional group (or several ones). In practice, however, it is often the internal allene carbon atom and/or the central methylene group of, e.g., 265 that carries substituents. Quite often

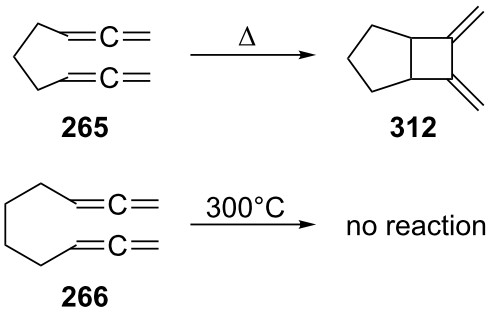

Scheme 72: The gas-phase pyrolysis of 1,2,7,8-nonatetraene (265) and 1,2,8,9-decatetraene (266).

methylene groups of the tether connecting the allene moieties are replaced by hetero atoms also, in particular by nitrogen and oxygen, as we shall see in Section 6.

A typical example for the above strategy has been described by Mukai and co-workers (Scheme 73) involving a Pauson-Khand reaction with the bisallene bissulfone $313\left(\mathrm{X}=\mathrm{CH}_{2}\right)$ [177]. On treatment with various rhodium catalysts under carbon monoxide it cyclizes in near quantitative yield to the 


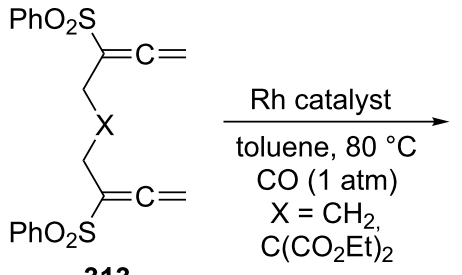

313

$\mathrm{SO}_{2} \mathrm{Ph}$<smiles>CCCCCC(CC)(C(=O)Oc1ccccc1)S(=O)(=O)Oc1ccccc1</smiles>

316<smiles></smiles><smiles>[X]C1CC(S(=O)(=O)Oc2ccccc2)=C2CCC2=C1S(=O)(=O)O</smiles>

314
315

(only for $\mathrm{X}=\mathrm{C}\left(\mathrm{CO}_{2} \mathrm{Et}\right)_{2}$ )<smiles>O=C1C=C2C(=C(S(=O)(=O)c3ccccc3)CCCCC2S(=O)(=O)c2ccccc2)C1</smiles>

$317(70 \%)$<smiles>O=C1CC2=C(C1)C(S(=O)(=O)c1ccccc1)CCCC=C2S(=O)(=O)c1ccccc1</smiles>

$318(23 \%)$

Scheme 73: Rh-catalyzed cyclization of a functionalized 1,2,7,8-nonatetraene.

bicyclo[5.3.0]decadienone compound $314\left(\mathrm{X}=\mathrm{CH}_{2}\right)$. The "allene dimerization product" 315 is not produced. With $\mathrm{X}=$ $\mathrm{C}\left(\mathrm{CO}_{2} \mathrm{CH}_{3}\right)_{2}$, however, 315 becomes a side product and, with certain rhodium catalysts, even the main product of the cyclization (up to $70 \%$ ). Heteroorganic variants of this process ( $\mathrm{X}=$ heteroatom) are known and will be discussed in Section 6. Nonsulfonylated bisallenes do not undergo this $[2+2+1]$ cycloaddition, which probably involves the intermediate generation of a rhodacycle. The $\mathrm{C}_{4}$-tethered derivative 316 behaves analogously, furnishing the bicyclic ketone derivatives $\mathbf{3 1 7}$ and $\mathbf{3 1 8}$. Even chain extension to five carbon atoms still permits some intramolecular bicyclization. Heating $\mathbf{3 1 6}$ in the absence of CO, but under the influence of [ $\left.\{\mathrm{RhCl}(\mathrm{CO}) \mathrm{dppp}\}_{2}\right]$, causes cyclization to the corresponding [3]dendralene derivative in excellent yield $(92 \%)[48,177]$.

A remarkable triple cyclization of two molecules of the 2,3allenoic acid 319 in the presence of one molecule of the parent bisallene 265 has recently been described by Ma and Lian
(Scheme 74) [178]. The reaction yields the tricyclic compound 320 in excellent yield considering what has been accomplished preparatively.

The group of Ma has revealed interesting cyclization reactions of various aliphatic and aromatic diketones derived from $\mathbf{2 4 2}$ and 265 (Scheme 75) [179]. For example, diketone 321 isomerizes under very mild conditions into the bridged furan derivative 322. Making the two substituents different, as in $\mathbf{3 2 3}$, causes the cyclization to provide two differently substituted furans, 324 and $\mathbf{3 2 5}$.

Many other multifunctionalized bisallenes derived from $\mathbf{2 6 5}$ have been cycloisomerized similarly and constitute an important high-yielding and multifaceted route to interesting bi- and polycyclic compounds [180-187], as the following examples show and with which we wish to conclude this section on cyclization reactions of $\alpha, \omega$-bisallenes. In Scheme 76 the first reaction displays a Rh-catalyzed cycloisomerization/dimeriza-

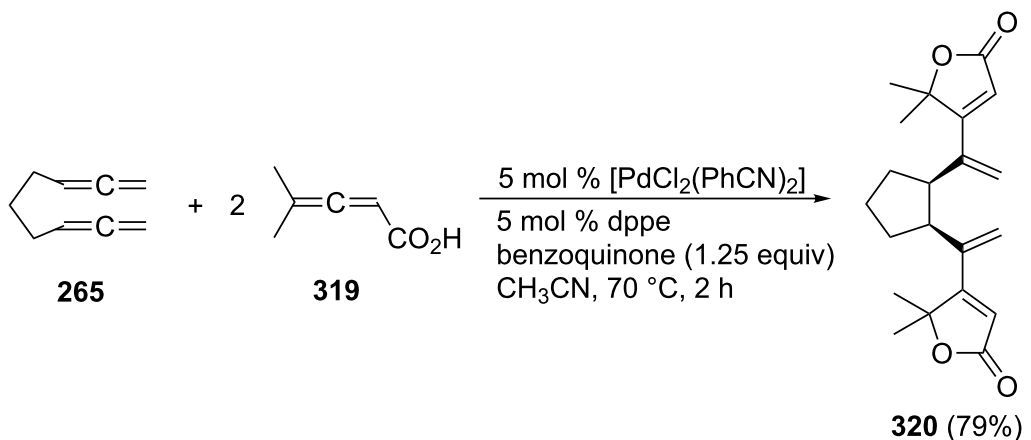


<smiles>[R]C(=O)C(=C=C)CCC(=C=C)C([R])=O</smiles>

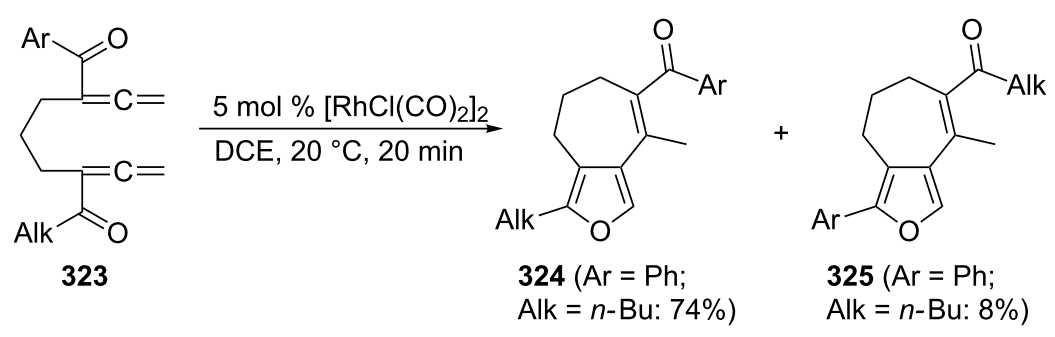

Scheme 75: Bicyclization of keto derivatives of 1,2,7,8-nonatetraene.

tion in which the diester $\mathbf{3 2 6}$ is converted into a product with the carbon skeleton of a steroid, $327[188,189]$. To obtain the high yield given in the scheme it is mandatory to reduce the concentration of $\mathbf{3 2 6}$ to low levels $(0.04 \mathrm{M})$. The second example concerns the lactonization of a bisallenic hydroxy acid 328, which leads to a macrocyclic bisallene 329, a pivotal intermediate for the preparation of various erythronolides (see Scheme 108 below); with $64 \%$ the yield of the process is remarkable [190].

A bisallene in which the two allene moieties are separated by no less than nine carbon atoms is the diketone 331, which is obtained from the diol 330 by a Dess-Martin oxidation, followed by propargylic isomerization on workup (column chromatography on silica gel; Scheme 77) [191]. Treatment of 331 with various Pd(II)-catalysts causes cyclization to different furanophanes, among them 332, which is produced in $10 \%$ yield. In fact, the spacer between the two carbonyl groups may be extended up to 14 methylene groups and the combined yields of the resulting macrocycles are still astonishingly high (up to $30-40 \%)$.

Since $\alpha, \omega$-bisallenes are bifunctional compounds, the question may be asked whether they could be used as monomers for the preparation of new polymeric materials. The answer is yes and not surprisingly it involves the more elongated bisallenes preferentially. For example, the diester 326, which has already been shown to be one of the work horses in intermolecular cyclization reactions (see above), was polymerized with an allylnickel catalyst [192]. The resulting polymer, which is soluble in

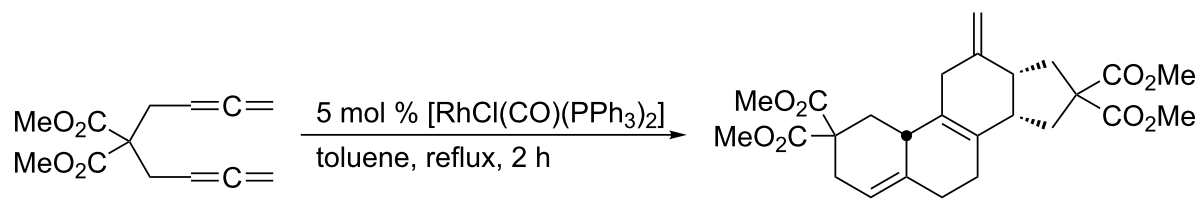

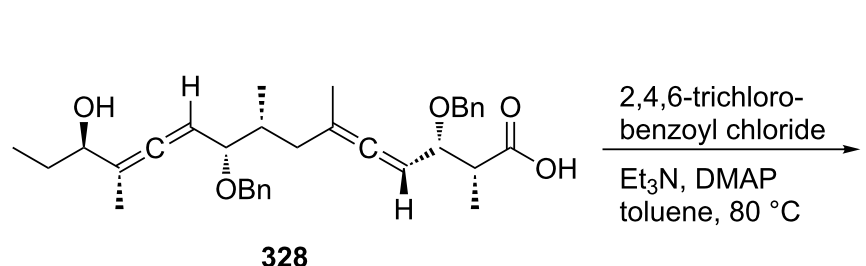

328

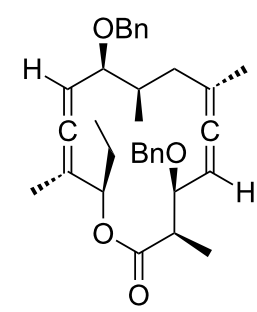

$329(64 \%)$ 


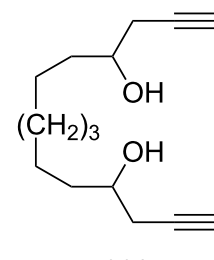

330

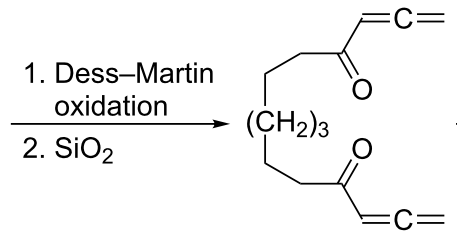

331

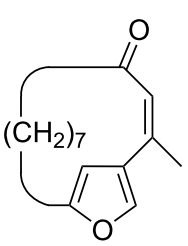

$332(10 \%)$

Scheme 77: Cycloisomerization of an $\alpha, \omega$-bisallene containing a $\mathrm{C}_{9}$ tether.

common organic solvents, is composed of an almost comparable content of 1,2 and 2,3-polymerized units. In another polymerization experiment, the $\alpha, \omega$-bisallenes $334\left(\mathrm{R}=-\left(\mathrm{CH}_{2}\right)_{4}\right.$, $-\left(\mathrm{CH}_{2}\right)_{6}-$ and - para- $\left.\mathrm{C}_{6} \mathrm{H}_{4}-\right)$ were subjected to a hydroboration experiment with thexylborane (333) and other organoboranes (Scheme 78) [193]. The obtained organoborane polymers 335 were submitted to chain-transformation reactions, leading, e.g., to the corresponding polyalcohols.

\section{Bisallenes with unsaturated spacer elements}

As delineated in the Introduction, unsaturated moieties can be used in many different ways to connect two allene units (see structures 8-12 in Figure 3).
One of the simplest unsaturated spacers is the carbon-carbon double bond. When interspersing it between two allene units $1,2,4,6,7$-octapentaene results, which may be present as the trans- or the cis-diastereomer, 337 [194] and 341, respectively (Scheme 79). Although the $E$-diastereomer 337 is indeed formed when the double dibromocyclopropanation product of $E$-1,3,5-hexatriene, i.e., the tetrabromide 336, is subjected to the DMS protocol (see above, Section 1.1), the overall yield of the reaction is low (32\%), and hydrocarbon $\mathbf{3 3 7}$ is not the only reaction product. For example, the five-membered-ring products $\mathbf{3 3 8}$ and $\mathbf{3 3 9}$ are also among the debromination products. The formation of cyclopentadiene-derived products, notably fulvenes, has often been noted in this transformation (see above) [26,194].

$$
\rangle_{333}{ }_{\mathrm{BH}_{2}}+=\mathrm{C}=\underset{\mathrm{R}}{334}=\mathrm{c}=\frac{\text { bulk }}{0{ }^{\circ} \mathrm{C}} \overbrace{335}
$$

Scheme 78: Organoborane polymers from $\alpha, \omega$-bisallenes.<smiles>C=C=CC=CC=C=CC1CC1(Br)Br</smiles>
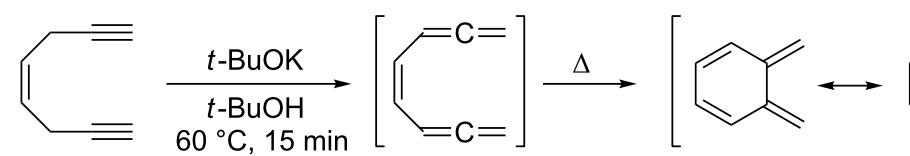<smiles>Ic1ccccc1</smiles>

340

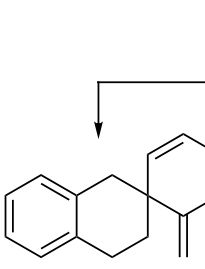

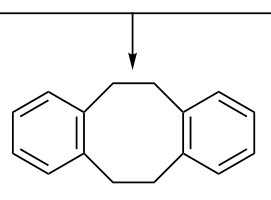

$345(2 \%)$

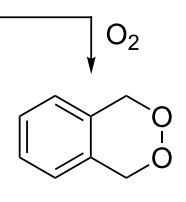

346 
The Z-diastereomer of 337, the pentaene 341, is apparently unknown as an isolated product up to the present day. It was postulated as early as 1963 by Sondheimer and Ben-Efraim to be generated from the diacetylene $\mathbf{3 4 0}$ by base treatment $(t$-BuOK, $t$-BuOH) [195]. That it actually had been generated in the above process was inferred from the isolation of two dimers, 344 and 345 , formed by a $[2+4]$ and a $[4+4]$ cycloaddition process, respectively. In the presence of oxygen the endoperoxide 346 is produced. The instability of $\mathbf{3 4 1}$ was rationalized by its rapid cyclization to $o$-xylylene, $\mathbf{3 4 2} / \mathbf{3 4 3}$, under the reaction conditions. A very similar process has been observed for those molecules in which the double bond in $\mathbf{3 4 1}$ has been replaced by a benzene or naphthalene ring as will be discussed below (see Section 4.1).

If the allene groups are not bonded to the ethylene unit in vicinal fashion, as in $\mathbf{3 3 7 / 3 4 1}$, but geminally, the hydrocarbon 349 results (Scheme 80 ). This cross-conjugated pentaene was prepared from its isomer 348 by a base-induced isomerization again, and the diacetylene was obtained by coupling the dibromide 347 with ethynylmagnesium bromide. The characterization of $\mathbf{3 4 9}$ rests on spectroscopic data as well as the formation of a Diels-Alder adduct when it is treated with tetracyanoethylene (TCNE) [196,197].

Several derivatives of $\mathbf{2}$ are known with interspersed acetylenic functions. One of the simplest hydrocarbons in this category is

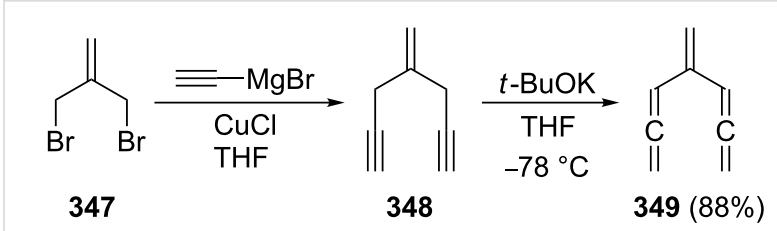

Scheme 80: The preparation of 4-methylene-1,2,5,6-heptatetraene (349).

351, which results when acetylene is bubbled through a solution of 1-bromo-3,3-dialkylallenes $\mathbf{3 5 0}$ in diethylamine in the presence of $\mathrm{Pd}\left(\mathrm{PPh}_{3}\right)_{4}$ and CuI (Scheme 81) [198]. The products $\mathbf{3 5 1}$ are crystalline solids, which are produced in acceptable yields (30-50\%). Analogously, 354 is produced (in yields up to $62 \%$ ) when 1,3-butadiyne (353) is employed as the coupling partner. Both 351 and 354 can be converted into dianions by treatment with $n$-butyllithium. The quench with trimethylsilyl chloride subsequently provided the respective bissilylated derivatives.

In a comparable, now Stille-type coupling reaction, Saalfrank and co-workers connected two molecules of $\mathbf{3 5 5}$ with bis(trimethylstannyl)acetylene (356) to furnish the diester derivative 357 (Scheme 82) [199].

More complex allenynes were prepared by Diederich and co-workers as building blocks for shape-persistent chiral alleno-

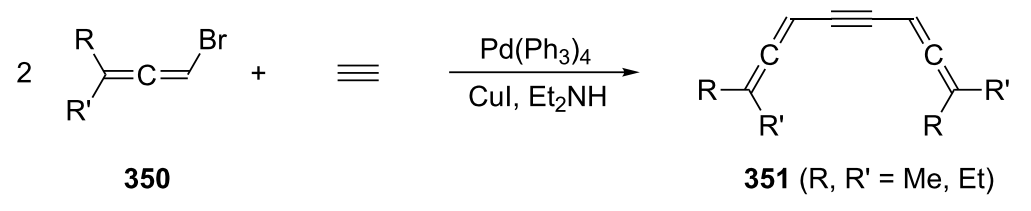

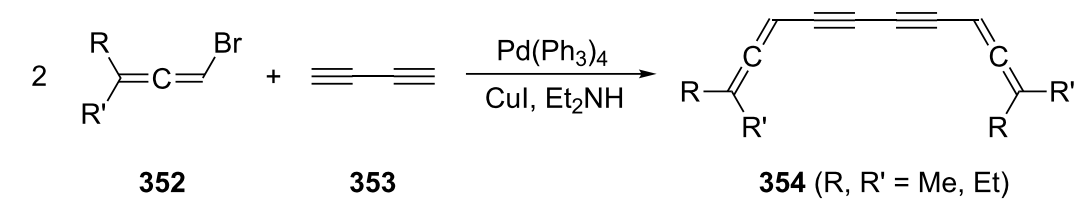

Scheme 81: The preparation of acetylenic bisallenes.

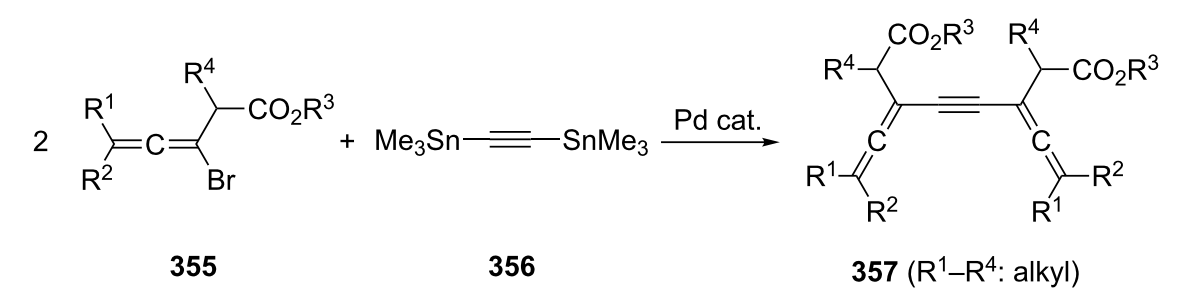


acetylenic macrocycles and cyclophanes. A typical example is shown in Scheme 83 [200]. By Pd-catalyzed coupling of the building blocks 358 and $\mathbf{3 5 9}$ the bisallene diyne $\mathbf{3 6 0}$ was first generated under propargylic rearrangement $\left(\mathrm{S}_{\mathrm{N}} 2^{\prime}\right)$. The protecting dimethylcarbinol function was then cleaved off by base treatment (so-called Favorskii cleavage) and the resulting terminal acetylene dimerized to 361 by Glaser coupling. In the final ring-closing step the tris(isopropylsilyl)-protecting groups were removed by fluoride treatment and the resulting $\alpha, \omega$ diacetylene was oxidatively cyclized by Eglinton coupling. The macrocyclic product 362 was produced in the form of two pairs of enantiomers and three achiral diastereomers.

Clearly, many more unsaturated spacer units can be designed (and prepared) and we would like to close this section with just one final example from the Roth group (Scheme 84) [146].
Grignard reaction of the dibromide 363 first led to the diacetylene 364 in very good yield, and base-catalyzed isomerization as above in Sondheimer's case (Scheme 79) provided in quantitative yield the bisallene 365 , in which the two allene moieties are separated by a 2,3-butadienyl unit. This bisallene is an interesting, highly unsaturated $\mathrm{C}_{10} \mathrm{H}_{10}$-hydrocarbon. On heating, for example, it cycloisomerizes to "tetramethylene benzene", 366, an unusual diradical that may be intercepted by double-bond dienophiles, such as fumarates, to furnish the $2: 1$ adduct $\mathbf{3 6 7}$ and the 2:2 product $\mathbf{3 6 8}$.

\section{Bisallene-containing ring systems 4.1 Aromatic bisallenes}

A relatively large number of compounds have been described in the chemical literature in which an aromatic system is connected to two (and sometimes more) allene moieties. It is

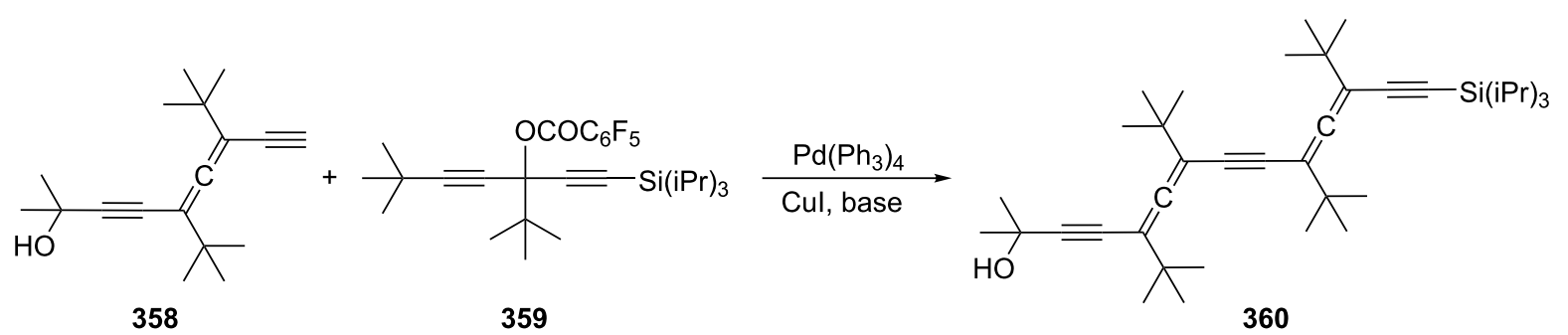

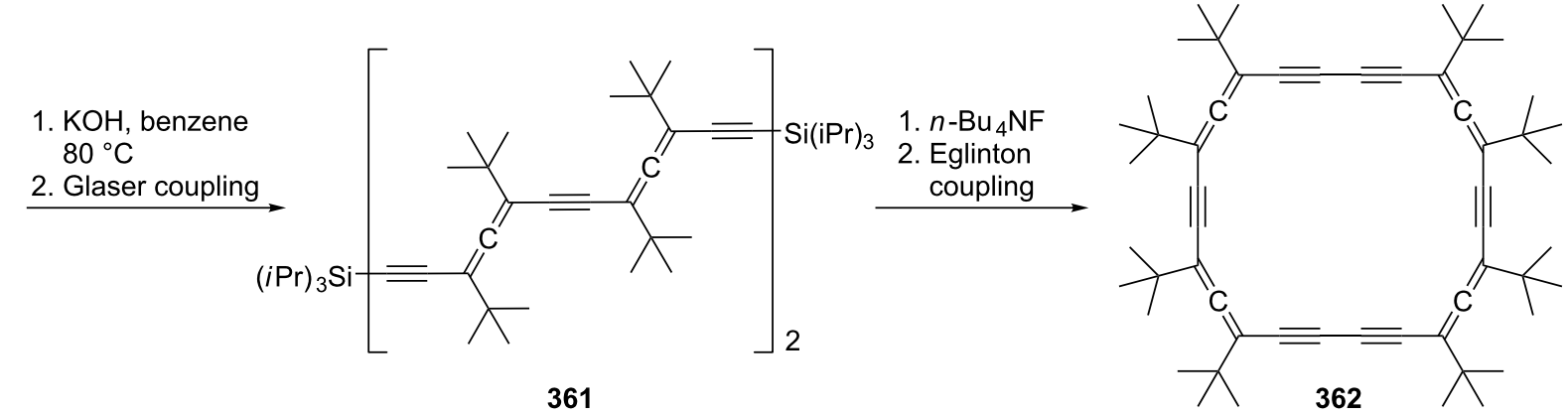

Scheme 83: The construction of macrocyclic alleno-acetylenes.<smiles>C=C(CBr)CBr</smiles>

363

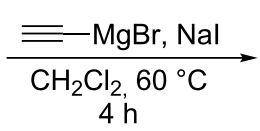

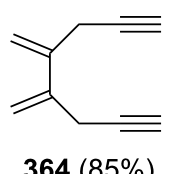

$364(85 \%)$

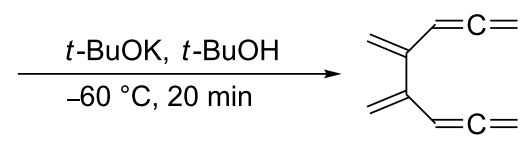

$365(90 \%)$

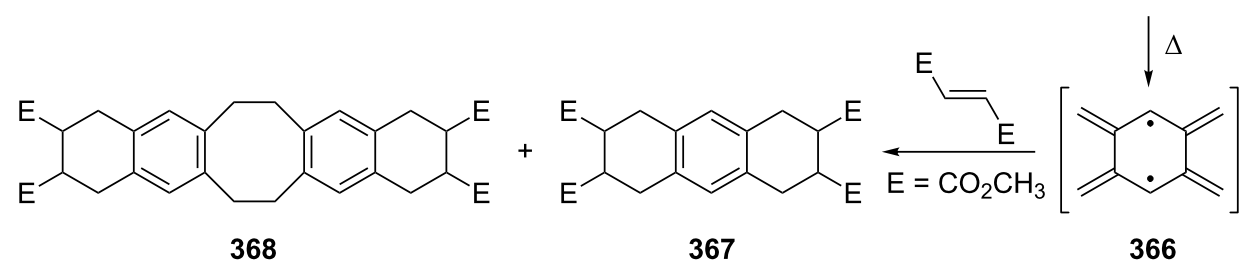

Scheme 84: Preparation and reactions of 4,5-bismethylene-1,2,6,7-octatetraene (365). 
not the purpose of this review to present all of these derivatives, but several typical examples will be discussed.

It is not surprising that the preparative methods we have come across several times so far are also extensively employed for the synthesis of aromatic bisallenes. For example, the ortho-bisallene $\mathbf{3 7 0}$ was prepared by both the base-catalyzed propargylic isomerization of the corresponding diacetylene $\mathbf{3 6 9}$ and the application of the DMS route to the ortho-divinylbenzene adduct 371 (Scheme 85) [201].

Hydrocarbon $\mathbf{3 7 0}$ can be isolated, i.e. it is considerably more stable than the "parent" system 341 (Scheme 79). Like its olefinic version 341 it can be trapped by oxygen to yield the endo-peroxide $\mathbf{3 7 3}$ or it dimerizes to a complex mixture of dimers $(47 \%)$ in which 372 forms one component. The next higher benzologue of 370, 2,3-bis(propadienyl)naphthalene, has also been prepared [201]. A more recent route to $\mathbf{3 7 0}$ involves the reduction of the appropriate bispropargylic diacetate with samarium diiodide in the presence of $\mathrm{Pd}\left(\mathrm{PPh}_{3}\right)_{4}$ [202]. A number of derivatives of $\mathbf{3 7 0}$ are known that play an important role in the studies of Braverman and co-workers on the generation of diradicals from bisallenic precursors (see Section 6 , hetero bisallenes).

1,4-Bis(propadienyl)benzene (376) and several of its derivatives have recently attracted the attention of a number of research groups. The parent hydrocarbon can be obtained easily by the DMS protocol from para-divinylbenzene (374) employing phase-transfer catalysis for the preparation of the tetrabromide intermediate 375 (Scheme 86) [203-206].

More often, though, modern metal-mediated coupling reactions are employed to prepare derivatives of $\mathbf{3 7 6}$. A general route involves different aromatic derivatives $\mathbf{3 7 7}$ and cross coupling of them with propargyl bromides 378 in the presence of $\mathrm{Pd}(0) /$ In to provide the aromatic polyallenes 379 , as illustrated in Scheme 87 [186,207]. A selection of allenic coupling prod-

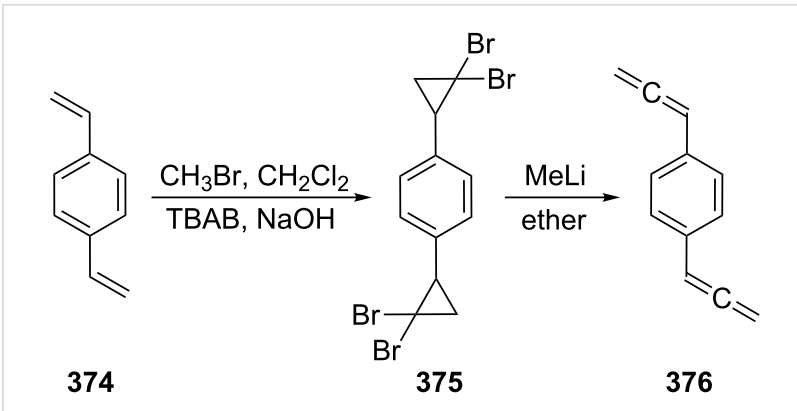

Scheme 86: The preparation of 1,4-bis(propadienyl)benzene (376).

ucts, 380-383, all produced in very good yields, is also shown in Scheme 87. 1,4-Bis(propadienyl)benzene derivatives with varying functional groups (i.e. ester or ether functions) have been prepared by using propargylic carboxylates as substrates [208,209].

Preparatively, the 1,4-bis(propadienyl)benzenes and their derivatives are of interest for several reasons. Reissig and co-workers demonstrated, for example, that the 1,4-bisallene 384 can be doubly cyclized in quantitative yield to the 3-pyrroline derivative $\mathbf{3 8 5}$ by stirring the allene precursor with silver nitrate in acetone (Scheme 88) [210].

Even if the allene groups are removed very far from the aromatic core, interaction between them can be induced as illustrated by the ring-closing metathesis reaction of $\mathbf{3 8 6}$. This hydrocarbon cyclizes in the presence of a Grubbs type I catalyst to the [15]paracyclophane $\mathbf{3 8 7}$ under high-dilution conditions (Scheme 89) [211]. We have noted a similar process already in Section 2.4 (Scheme 77) employing a completely saturated tether (but containing two carbonyl groups) between the allene groups.

A more complex case, reported by Krause, Vögtle and co-workers, concerns the trisallene $\mathbf{3 8 8}$, which contains the 1,4bis(propadienyl)benzene motive twice; it was assembled from

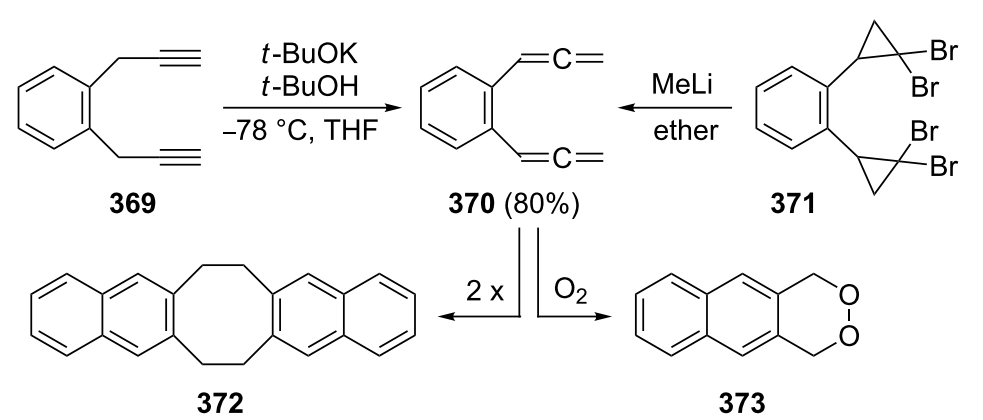

Scheme 85: Preparation of 1,2-bis(propadienyl)benzene (370). 


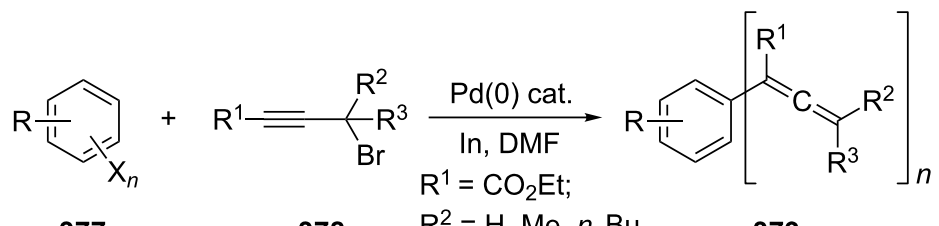

377

$(\mathrm{X}=\mathrm{Br}, \mathrm{I}, \mathrm{OTf}$

$\mathrm{R}=\mathrm{Me}, \mathrm{MeO}, \mathrm{NO}_{2}$ etc.

378

$\mathrm{R}^{2}=\mathrm{H}, \mathrm{Me}, n-\mathrm{Bu}$

$\left(R^{3}=H\right)$<smiles>C=C=C(CC)c1ccc(C(=C=C)CC)cc1</smiles><smiles>C=C=C(C)c1ccc(C(C)=C=C)nc1</smiles>

$380(84 \%)$
$381(65 \%)$<smiles>C=C=Cc1ccc(-c2ccc(C=C)cc2)cc1</smiles>

$382(90 \%)$<smiles>C=C=C(C)c1cc(C(C)=C=C)cc(C(C)=CC)c1</smiles>

$383(70 \%)$

Scheme 87: The preparation of aromatic and heteroaromatic bisallenes by metal-mediated coupling reactions.

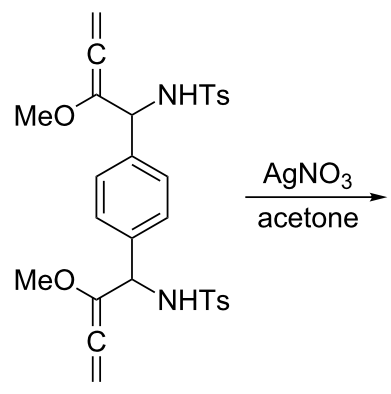

384<smiles>COC1=CCN(C)C1c1ccc(C2C(OC)=CCN2[AsH])cc1</smiles>

385 (94\%)
Scheme 88: Double cyclization of an aromatic bisallene.

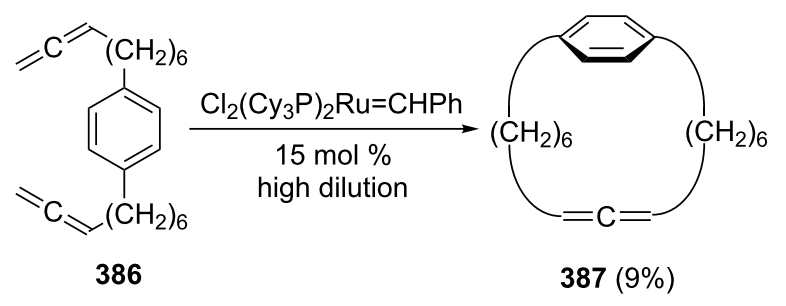

Scheme 89: Preparation of an allenic [15]paracyclophane by a ringclosing metathesis reaction of an aromatic $\alpha, \omega$-bisallene.

smaller building blocks and connected to the macrocyclic alcohol 389 by treatment with KN(TMS) 2 (Scheme 90) [212]. Manipulation of the propargyl alcohol section of $\mathbf{3 8 9}$ by standard methods ultimately provided the $\left[3_{4}\right]$ allenophane 390 [213].
Finally, aromatic bisallenes are interesting substrates for the preparation of new polymeric materials. For example, the disulfides 391 have been copolymerized with 1,4-bis(propadienyl)benzene (376) to yield the soluble conjugated polymer 392 (Scheme 91) [193]. And a Pd-catalyzed three-component coupling polymerization of $\mathbf{3 7 6}$ and various difunctionalized aromatics in the presence of nucleophiles $\left(\mathrm{Nu}^{-}\right)$yielded polymer 393, a new type of substituted poly(arylene-vinylene) [214].

An alternating boration/copolymerization between 376 and 2,7diethynylfluorene (395) was realized by first carrying out a selective haloboration of $\mathbf{3 7 6}$ and then copolymerizing the generated intermediate 394 with 395 (Scheme 92) [189]. When the copolymer 396 was doped with base, it was converted into a polyanion, which may possess $\sigma / \pi$-conjugated-polymer character.

The number of bisallenes in which the allene units are connected to a heteroaromatic ring system seems to be very small [215].

In closing we note that a new class of aromatic bisallenes has recently been reported in which the allene units are not bonded to a planar aromatic compound but to a layered one. The case in point is provided by the bisallene 399 , which has been prepared from the [2.2]paracyclophane diol 397. When this is treated with perchloromethylmercaptane (PCMM) in dichloromethane at low temperature, the bispropargyl intermediate $\mathbf{3 9 8}$ is formed 

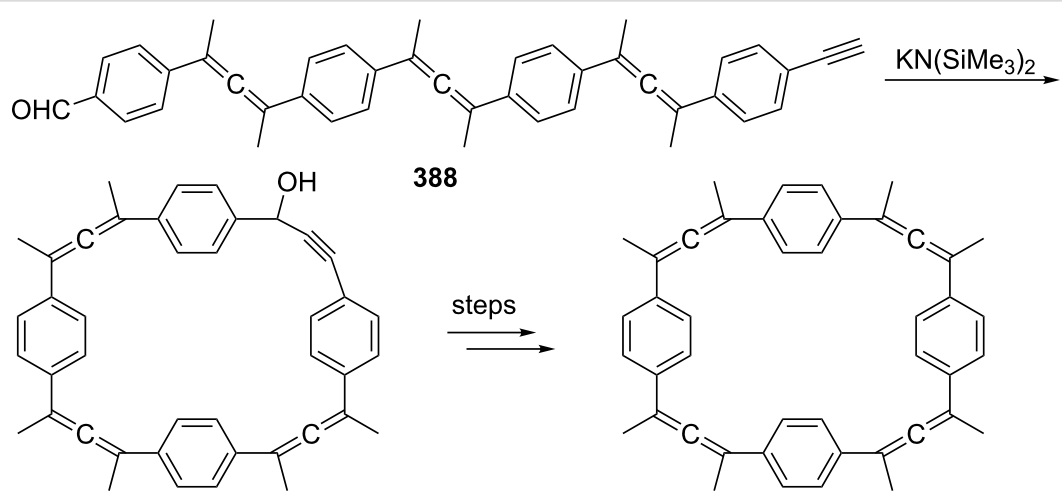

388
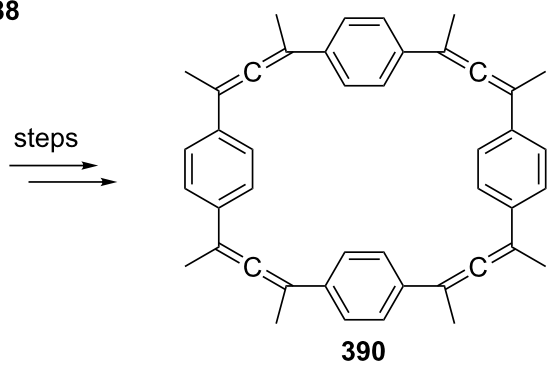

390

Scheme 90: Preparation of a macrocyclic ring system containing 1,4-bis(propadienyl)benzene units.

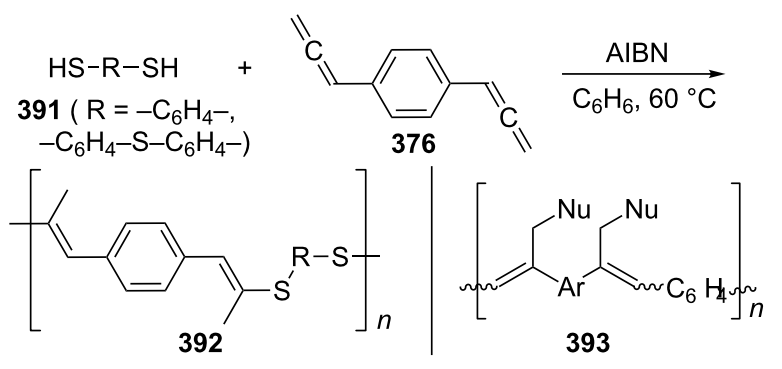

Scheme 91: Preparation of copolymers from 1,4bis(propadienyl)benzene (376). first, which subsequently undergoes a rapid [2.3] sigmatropic shift to 399 in very good yield (Scheme 93) [216,217].

\subsection{Bisallenes connected by cyclic spacer groups}

There is a countless number of ways by which two (and occasionally more) allene groups may be connected by saturated ring systems and some of these have been discussed in the Introduction already (see above). Here we will begin our review with those compounds in which the two allene moieties are anchored in the ring system in semicyclic fashion. We start with these derivatives for historical reasons and because of their structural simplicity.

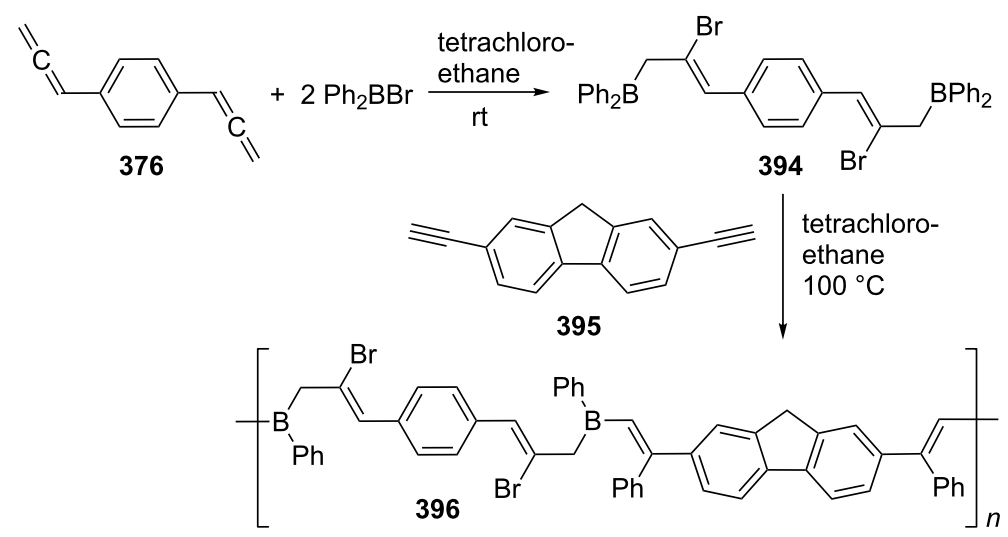

Scheme 92: A boration/copolymerization sequence of an aromatic bisallene and an aromatic bisacetylene.

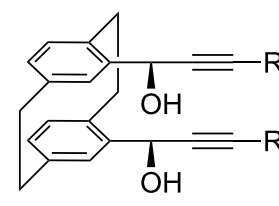

$397(\mathrm{R}=n-\mathrm{Pr}, n-\mathrm{Bu})$

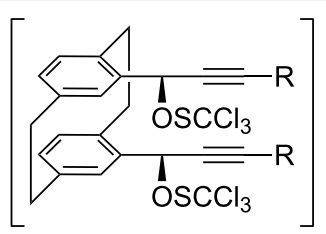

398

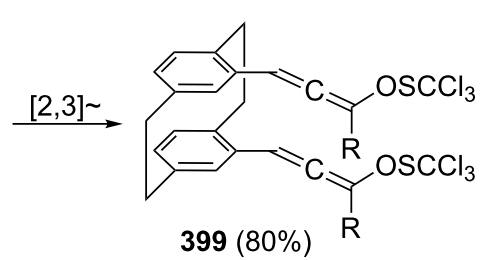

$399(80 \%)$

Scheme 93: Formation of a layered aromatic bisallene. 


\subsubsection{Semicyclic bisallenes}

The simplest semicyclic bisallene is hydrocarbon $\mathbf{4 0 0}$, bis(vinylidene)cyclopropane (Figure 4). Taking this compound as a starting point, we will discuss the homologous series with 401 and 402 as the next members in the series. From 401 onwards, the possibility of structural isomers exists, since the allene groups may be positioned in a neighboring or any other arrangement. Whereas $\mathbf{4 0 0}$ apparently has not been synthesized yet, and its heterorganic variants in which the ring methylene group has been replaced by oxygen, sulfur or nitrogen functions are also unknown, the four-membered ring compound $\mathbf{4 0 1}$ and its derivatives have been known and studied for a while.<smiles>C=C=C1CC1=C=C</smiles>

400<smiles>C=C=C1CCC1=C=C</smiles>

401<smiles>C=C=C1CCCC1=C=C</smiles>

402
Figure 4: The first members of the semicyclic bisallene series.

First derivatives of $\mathbf{4 0 1}$ were obtained by Hartzler in his classical studies on the higher cumulenes [218,219]. Having prepared the fully tert-butylated hexapentaene $\mathbf{4 0 3}$ for the first time he studied, among other things, its $[2+2]$ cycloadditions with activated double and triple bonds. The addition of tetrafluoroethylene (404) resulted in the formation of the bis(vinylidene)cyclobutane 405 (Scheme 94). Reactive olefins undergo cycloaddition only to the central double bond of $\mathbf{4 0 3}$. Interestingly, ethylene itself did not react with the cumulene; in a corresponding experiment $\left(200^{\circ} \mathrm{C}\right.$, EtOAc) only the starting material $\mathbf{4 0 3}$ was isolated at the end of the process.

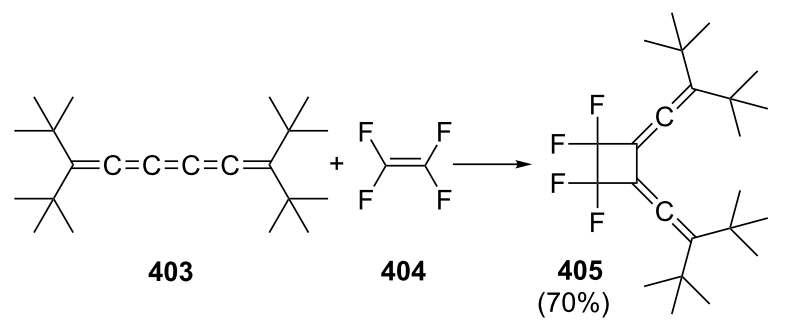

Scheme 94: Preparation of the first bis(vinylidene)cyclobutane derivative.

In their studies on cumulenic carbenes, Stang and co-workers observed that the [3]cumulene $\mathbf{4 0 6}$ dimerized spontaneously at $-20{ }^{\circ} \mathrm{C}$ to the bisallenic spiro compound 407 , whose structure as a $s y n$-head-to-head dimer was established by an X-ray struc- tural study. Analogously, the pentatetraene 408 leads to 409 quantitatively when left at room temperature for three days (Scheme 95) [220-222]. It is likely that a part of the driving force of these dimerizations is provided by the strain of the three-membered ring. Another derivative of type 409 is produced when tetraphenylpentatetraene is thermally dimerized: the resulting hydrocarbon $\mathbf{4 1 0}$ undergoes an interesting thermal rearrangement to $\mathbf{4 1 1}$ when heated to $80^{\circ} \mathrm{C}$ [223].

2<smiles>CC(C)=C=C=C1C2CCCCC12</smiles>
$\stackrel{-20{ }^{\circ} \mathrm{C}}{\longrightarrow}$ 406<smiles>CC(C)=C=C1CC2(C1=C=C(C)C)C1CCCCC12</smiles>

407<smiles>CC(C)(C)C=C=C=C=C1C(C)(C)C1(C)C</smiles>

408<smiles>CC(C)(C)C=C1C(=C=C2C(C)(C)C2(C)C)C1=C=C1/C(=C/C(C)(C)C)C(C)(C)C1(C)C</smiles><smiles>C(=C(C1=CC(=Cc2ccccc2)C(=C(c2ccccc2)c2ccccc2)C1=C(c1ccccc1)c1ccccc1)c1ccccc1)=C(c1ccccc1)c1ccccc1</smiles>

410<smiles>C(=C(c1ccccc1)c1ccccc1)C1=C(c2ccccc2)c2c1c(C(c1ccccc1)c1ccccc1)c1ccccc1c2-c1ccccc1</smiles>

Scheme 95: Dimerization of strain-activated cumulenes to bis(vinylidene)cyclobutanes.

For compounds such as $\mathbf{4 0 7}$ and $\mathbf{4 0 9}$ there always exists in principle the question of whether these products are head-to-head (1,2-vinylidene) or head-to-tail (1,3-vinylidene) dimers. Additionally, it could be the central double bond of the substrate which reacts, giving rise to the formation of a [4]radialene [224,225]. Whereas the above dimerizations are clearly of the 1,2-type, 1,3-dimers have also been described. Thus, both tetraphenyl-1,2,3-butatriene (412) and 1,1-diphenyl-4,4bis(trifluoromethyl)-1,2,3-butatriene (414) photodimerize in the solid state to the 1,3-dimers 413 and $\mathbf{4 1 5}$, respectively (Scheme 96) [226,227].

The two parent hydrocarbons 1,2- and 1,3-bis(vinylidene)cyclobutane, $\mathbf{4 2 1}$ and $\mathbf{4 2 0}$ respectively, were eventually synthesized by first thermally dimerizing allene, 416, to a mixture of 1,2-bismethylenecyclobutane (418, main product) and its 1,3-isomer, 421, and then subjecting this mixture to the DMS 


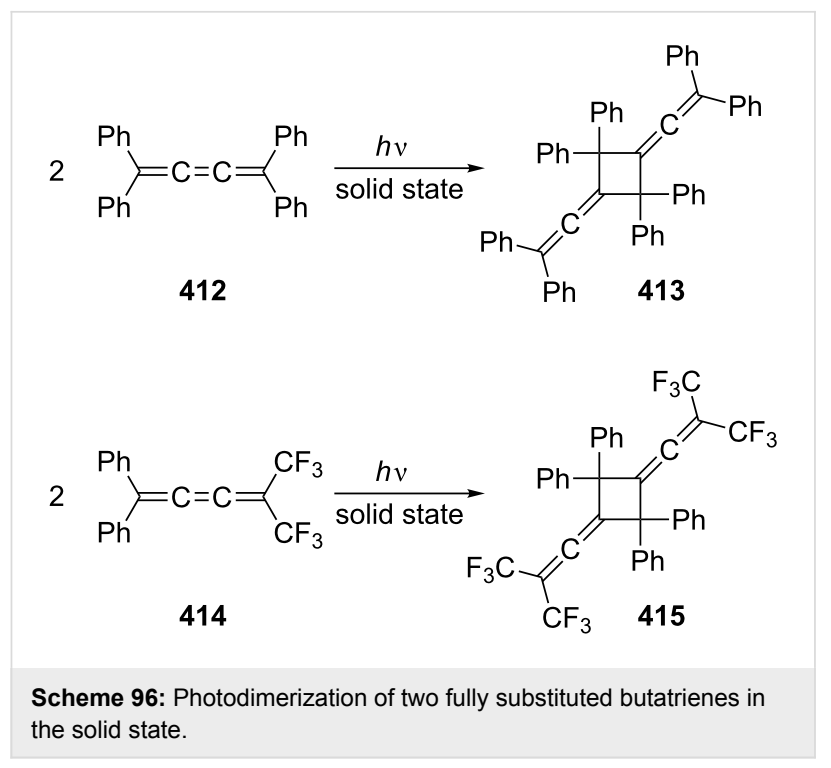

allenation protocol (Scheme 97) [228]. The two hydrocarbons can be distinguished by their spectroscopic data and, additionally, by a cycloaddition experiment with dimethyl acetylenedicarboxylate: only the 1,2-dimer $\mathbf{4 2 1}$ affords a cycloadduct, the benzocyclobutenophane $\mathbf{4 2 2}$. More recently it was suggested that 421 can also be generated from the biscarbonate 419 by treating it with $\left(\eta^{2}\right.$-propene $) \mathrm{Ti}(\mathrm{OiPr})_{2}$; the yield of the bisallene is poor, though, and it has been identified only in situ by ${ }^{1} \mathrm{H}$ NMR spectroscopy [229]. We will return to this method in the context of higher homologues of $\mathbf{4 2 1}$ (Scheme 99).

Both isomers of bis(vinylidene)cyclopentane have been prepared: the 1,2-isomer by application of the route just discussed (isolated yield: 45\%) [229] and the 1,3-isomer $\mathbf{4 2 4}$ by the DMS method from 1,3-bismethylenecyclopentane (423) (Scheme 98) [230]. The intended thermal isomerization of $\mathbf{4 2 4}$ to $\mathbf{4 2 5}$ by flash vacuum pyrolysis could not be realized. Rather than yielding this "nor[5]radialene" the pyrolysis provided the dienyne 427, presumably via the "half-isomerized" intermediate $\mathbf{4 2 6}$ by a symmetry-allowed 1,5-hydrogen shift.

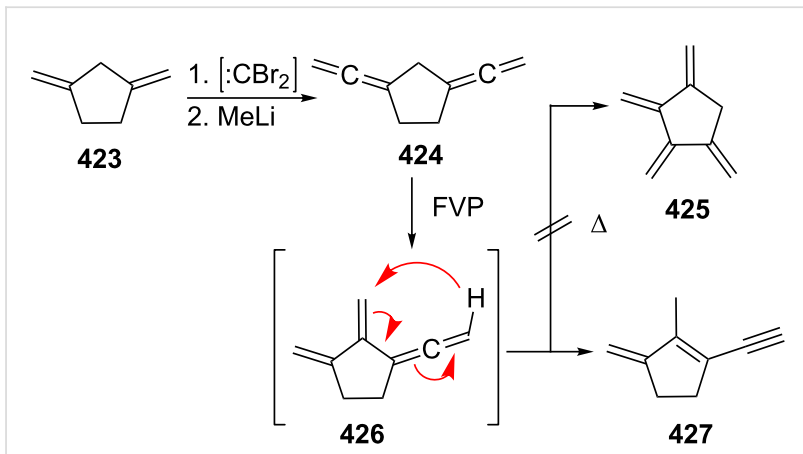

Scheme 98: The preparation of 1,3-bis(vinylidene)cyclopentane and its thermal isomerization.

Proceeding to the corresponding bis(vinylidene)cyclohexanes, we note that three isomers are conceivable now and that the problem of preparing all three has been addressed in the chemical literature already (Scheme 99) [230]. However, when the Sato method was applied to the bisether $\mathbf{4 2 8}$, only the bicyclic bismethylenecyclobutene $\mathbf{4 3 0}$ resulted, although in very good yield. A similar result was observed for the next higher homologue, 1,2-bis(vinylidene)cycloheptane. Evidently, the particular arrangement of the allene units in the relatively unstrained ring of $\mathbf{4 2 9}$ makes interaction between them particularly favorable. In the cases of the 1,3 and 1,4-isomers, 432 and 436 respectively, their preparation from the starting dienes $\mathbf{4 3 1}$ and $\mathbf{4 3 5}$ posed no problems. Whereas the thermal isomerization of $\mathbf{4 3 2}$ caused isomerization to a mixture of the dienynes $\mathbf{4 3 3}$ and $\mathbf{4 3 4}$, which are themselves in thermal equilibrium by a 1,3-hydrogen shift, the thermal rearrangement of $\mathbf{4 3 6}$ indeed provided a 1,2,3,4-tetramethylenecyclohexane (437), formally a 2,5bridged [4]dendralene [48]. The latter hydrocarbon is in thermal equilibrium with the cyclobutene isomer 438, a bicyclic

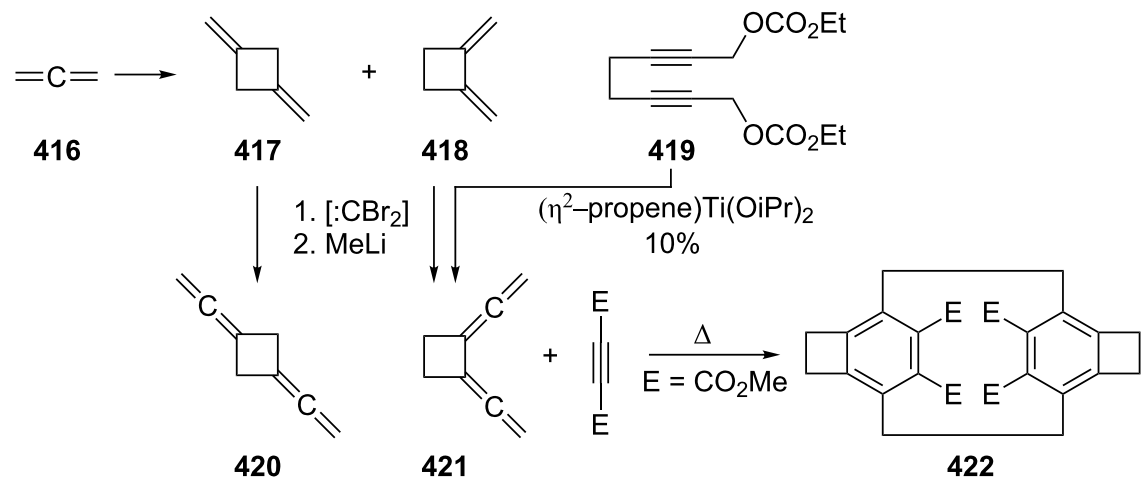

Scheme 97: Preparation of the two parent bis(vinylidene)cyclobutanes. 


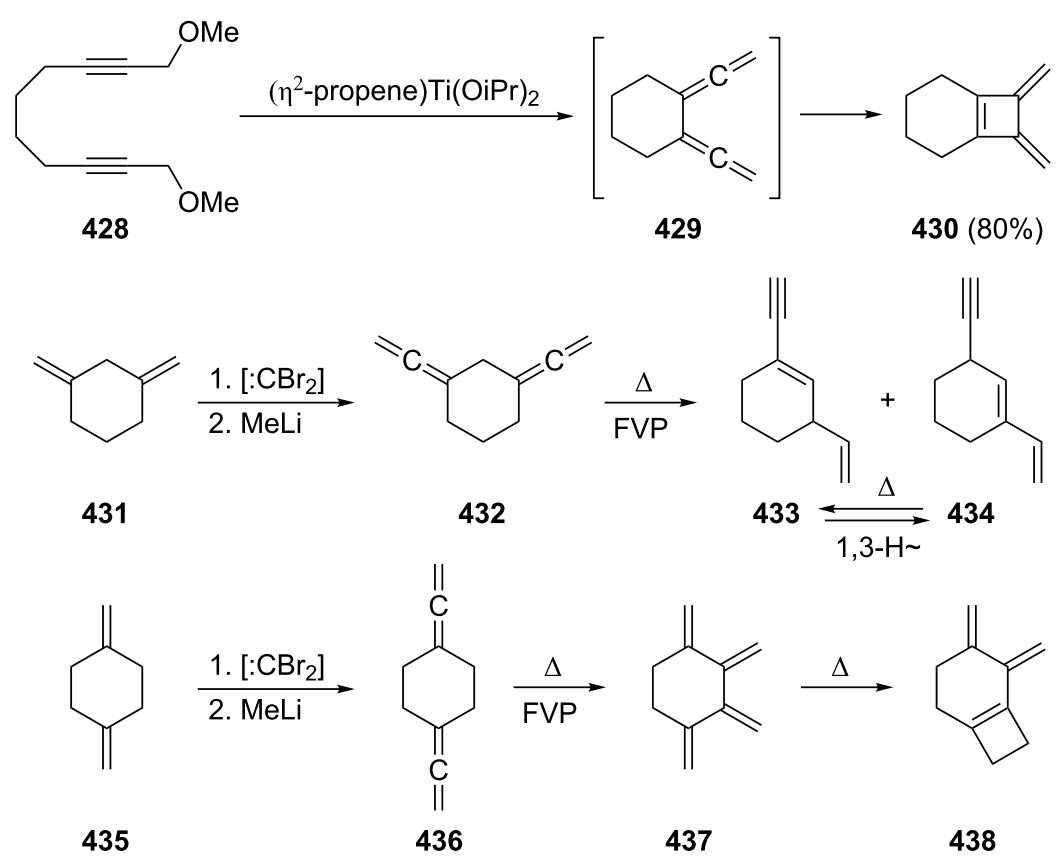

Scheme 99: The preparation of the isomeric bis(vinylidene)cyclohexanes.

[3]dendralene. The structure of both products was established by spectroscopic and chemical means. The thermal isomerization of $\mathbf{4 3 6}$ also sheds some light on the rearrangement of 1,5,9cyclododecatriyne into [6]radialene, as will be discussed in Section 5 (Scheme 113) [231].

More complex oligocyclic spacer groups have been inserted between the allene moieties as shown by the examples in
Scheme 100. Both 439 and 444 were obtained from the corresponding dienes by the DMS route (see Section 1.1). In an attempt to prepare a stable divinylperoxide, 440, Schuster and Mebane [232] added singlet oxygen to the bicyclic bisallene 439. However, rather than isolating the expected adduct $\mathbf{4 4 0}$, the two rearrangement products $\mathbf{4 4 2}$ and $\mathbf{4 4 3}$ were obtained. The process probably involves the diradical intermediate $\mathbf{4 4 1}$, generated by the homolysis of the oxygen-oxygen bond under<smiles>C=C1C(=C)C2CCC1C2=O</smiles>

439

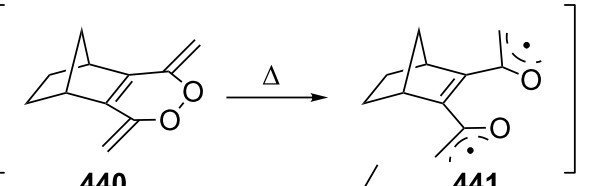

441<smiles>C=C1OCC(=O)C2CCC1C2</smiles>

$442(29 \%)$

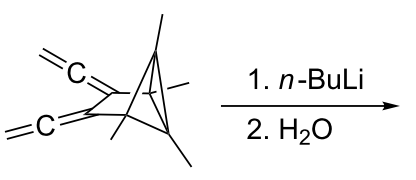

444<smiles>C#Cc1c(C)c(C)c(C)c(C#C)c1C#C</smiles>

445 
the reaction conditions. With TCNE, on the other hand, 439 provided the corresponding $[2+4]$ cycloadduct. The bisallene 444 was prepared by Hogeveen and Heldeweg and produced the expected Diels-Alder adduct with TCNE. They noted that under the conditions of its preparation from the corresponding dichlorocarbene bis-adduct, the diethynylbenzene derivative $\mathbf{4 4 5}$, an aromatic isomer of $\mathbf{4 4 4}$, is also produced as a secondary product [233].

That these highly strained hydrocarbons are prone to reduce their angular strain by undergoing rearrangement processes is also underlined by an example of Hashmi and Szeimies who reported that the [1.1.0]bicyclobutane derivative $\mathbf{4 4 6}$ cannot be isolated and participates in an intramolecular ene reaction to provide the more stable isomer 447 (Scheme 101) [234].

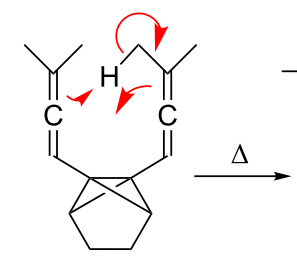

446

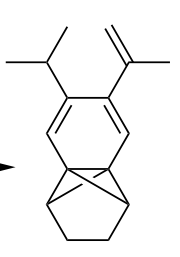

447

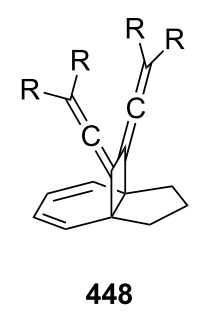

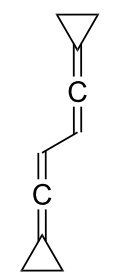

449

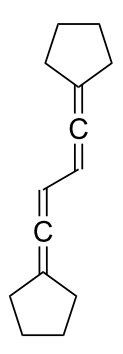

450

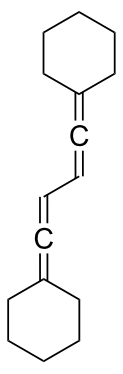

451
Scheme 101: A selection of polycyclic bisallenes.

In closing this section we note that many other types of semicyclic bisallenes can be designed on the drawing board that should possess interesting structural and chemical properties including the (still unknown) cyclopropane derivative 449.
Compounds of this general type, in which the three-membered rings are replaced by five- and six-membered rings, $\mathbf{4 5 0}$ and 451 respectively, have been prepared though [42,58-64]. The four-membered analogue of $\mathbf{4 4 9}$ is also unknown. Another semicyclic bisallene of type $\mathbf{4 3 9} / \mathbf{4 4 4}$, the fully substituted derivative 448 was obtained by Tobe et al. as a side product in a study concerned with the preparation of cyclo $[n]$ carbons by cycloreversion reactions from propellane-annelated dehydro[n]annulenes [235].

\subsubsection{Endocyclic bisallenes}

In their simplest form endocyclic bisallenes are those compounds that contain both allene units in one ring system, as represented by structure $\mathbf{1 5}$ in Figure 3. The allene units may be directly bonded to each other (conjugated) or nonconjugated (positioned in any section of the ring system). A sizable number of these compounds have been prepared during the past few decades and studied especially from the structural viewpoint.

The first author to investigate endocyclic, alicyclic bisallenes apparently was Skattebøl, who, in 1961, prepared hydrocarbons 453 and 455 from the respective precursors 452 and 454 by methyllithium treatment (Scheme 102) [236]. Both hydrocarbons were obtained in good (isolated) yields. Since any 1,3disubstituted allene is chiral, the products 453 and 455 should have been formed as mixtures of diastereomers, a meso- and a $d, l$-form. However, the first publication did not address this question. It was, on the other hand, soon discovered that $\mathbf{4 5 3}$ undergoes a thermal isomerization to 2,3-divinyl-1,3-cyclohexadiene (456), a bridged [4]dendralene [48], which is produced in virtually quantitative yield [237].

From that time, the early 1960 s, the questions of the detailed stereostructures of endocyclic bisallenes and their reactivity, particularly the behavior of $\mathbf{4 5 3}$, have interested many authors. Dehmlow and Stiehm later performed a thorough stereochemical analysis of the tetrabromides $\mathbf{4 5 2}$ (and their dibromide precursors) and suggested that $\mathbf{4 5 3}$ has the meso-, $\mathbf{4 5 7}$, and not the $d$,l-configuration, 458 (Figure 5) [238,239]. This suggestion was confirmed later by an X-ray structural analysis by

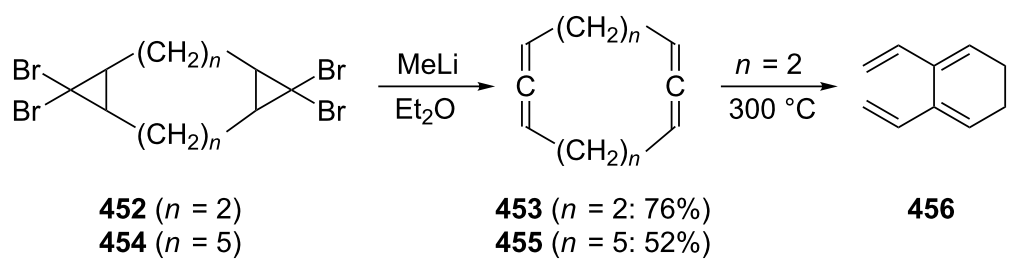


Irngartinger [240] and a thorough dynamic NMR analysis (DNMR) by Anet and co-workers [241]. According to Anet's analysis, the lowest energy conformation of $\mathbf{4 5 3}$ has $C_{i}$ symmetry. Symmetrization of this conformation (457) results in a $C_{2 h}$ time-average symmetry; the experimentally determined barrier of this conformational change by DNMR spectroscopy leads to a $\Delta G^{\ddagger}$ of $7.1 \mathrm{kcal} / \mathrm{mol}$. The ground state of $\mathbf{4 5 8}$ is found by force-field calculations to possess a "nonintersecting" twofold axis of symmetry [242]. Apparently the $d, l$-diastereomer $\mathbf{4 5 8}$ is unknown until the present day.<smiles>C1=CCCC=C=CCCCC=1</smiles>

457

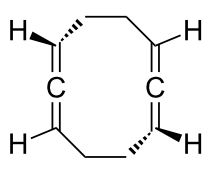

458
Figure 5: The stereochemistry of 1,2,6,7-cyclodecatetraene.

A number of other cyclobisallenes have been described in which the connecting bridges are of different length; most of these hydrocarbons were obtained by application of the DMS protocol to the corresponding dienes. Scheme 103 collects some of these compounds. Whereas the isomer 459 of the above decatetraene 453 could be isolated, its isomeric hydrocarbon 460 valence tautomerized to the bridged bismethylenecyclobutene 461 (for the bisallene to bismethylenecyclobutene isomerization of acyclic bisallenes, see Section 1.4.1) [243]. In a (rare) route not involving the DMS synthesis, Keese and Boss subjected propargylic acetates, such as $\mathbf{4 6 2}$, to a double methylation reaction with $\mathrm{LiCuMe}_{2}$ in ether at $-15{ }^{\circ} \mathrm{C}$ (Scheme 103). Depending on the configuration of the substrate 462 (meso or $d, l)$ the meso-bisallene 463 or its $d, l$-diastereomer 464 were obtained in excellent yields. This route can also be applied to the preparation of other derivatives of 1,2,6,7-cyclododecatetraene [155].

To prepare the meso- (467) and the $d, l$-diastereomers $(\mathbf{4 6 8})$ of the 4,9-dimethyl derivatives of 1,2,6,7-cyclodecatetraene $\mathbf{4 5 3}$, Roth and co-workers first catalytically dimerized (E)-1,3-pentadiene (465) to the 1,5-cyclooctadiene derivative 466 which, on DMS treatment, provided a mixture of the two stereoisomers in 70:30 ratio (Scheme 104) [244]. The two compounds could be separated by gas chromatography; if the cyclopropylidene-toallene conversion was carried out in the presence of spartein, 468 was obtained in optically active form. On pyrolysis $\left(300{ }^{\circ} \mathrm{C}\right)$ the above conversion to 2,3-divinyl-1,3-cyclohexadiene derivatives (and their follow-up products) took place. It was concluded from trapping experiments and a stereochemical analysis [245-248] that the meso-compound isomerizes by a concerted Cope-type rearrangement via a diradical intermediate, whereas the pyrolysis of the $d, l$-compound involves two competing reactions, a concerted and a nonconcerted one. The different behavior of the two diastereomers was traced back to the boat and chair geometries of the respective transition states.

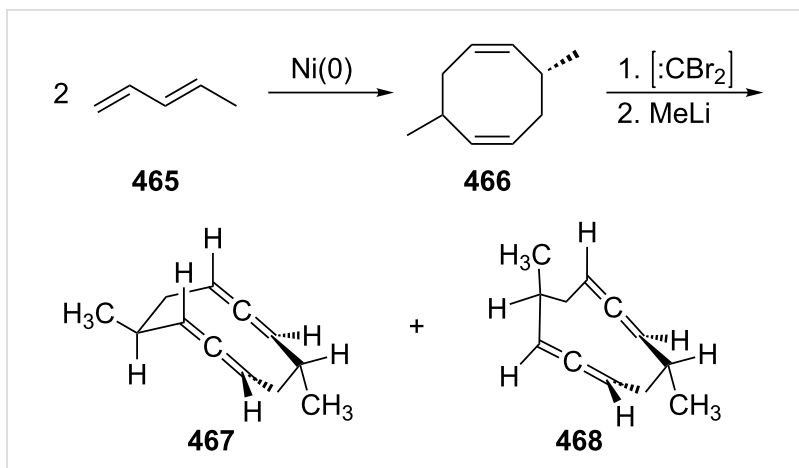

Scheme 104: Synthesis of diastereomeric derivatives of $1,2,6,7-$ cyclodecatetraene.<smiles>C1=CCCC=C=CCCC=1</smiles>

459

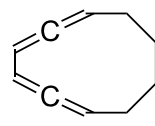

460

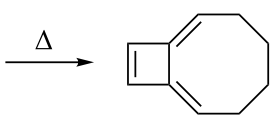

461

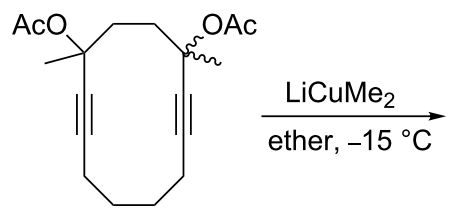

462

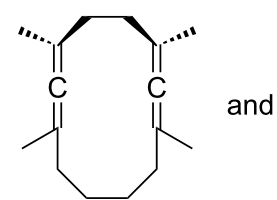

463

(meso, 90\%)

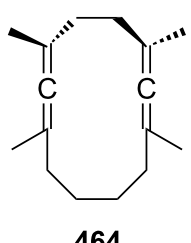

464

$(d, I, 91 \%)$ 
A number of functionalized derivatives of cyclic bisallenes have also been described. For example, as demonstrated in Scheme 105, on metalating 1,8-cyclotetradecadiyne (469), with $n$-butyllithium in THF, a polyanion is generated, which, after quenching with $\mathrm{TMSCl}$, furnishes three products, among them the cyclic bisallene derivative 470 [249].

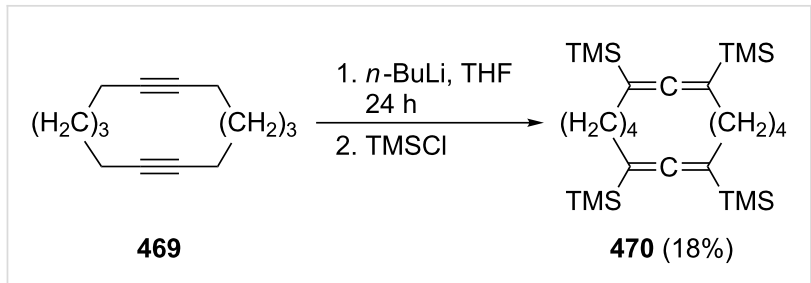

Scheme 105: Preparation of a derivative of 1,2,8,9-cyclotetradecatetraene.

A number of derivatives are known in which the usually saturated part of a cyclic bisallene (see 15, Figure 3 ) carries a functional group. For example, the Sondheimer group has obtained the two diketones $\mathbf{4 7 5}$ and $\mathbf{4 7 6}$ by first preparing the diketals 472 and 473 from the corresponding diolefin 471 by the DMS route and then hydrolyzing them to the target molecules (Scheme 106) [250,251]. When the allene synthesis was carried out in the presence of (-)-sparteine the two ketals were obtained in optically active, 472, and inactive form, 473. The two diastereomers could be separated by column chromatography on silica gel and subsequent hydrolysis yielded the optically active diketone $(+)-\mathbf{4 7 5}$ and its inactive stereoisomer $\mathbf{4 7 6}$ (meso) [252]. In several preparative applications of both $\mathbf{4 7 2 / 4 7 3}$ and 475/476 the Sondheimer and Garrat groups exploited the potential of these cyclic bisallenes for further ring expansions. Thus, by several steps the ketals were converted into the fourteenmembered allenic ketones $\mathbf{4 7 4}$, which, again, were obtained as $d, l$ - and meso-diastereomers [252].

Later studies saw the use of these diketals for the preparation of even more extended cumulenic systems. Whereas the monocyclic dicumulenic dione 477 could be prepared by application of the above protocol [253], the attempted preparation of $3,4,5,6,11,12,13,14$-cyclohexadecaoctaen-1,9-dione from the cyclic bis-allenic precursor $\mathbf{4 7 9}$ failed, since this bisketal could not be obtained from its precursor $\mathbf{4 7 8}$ (Scheme 107) [254,255].

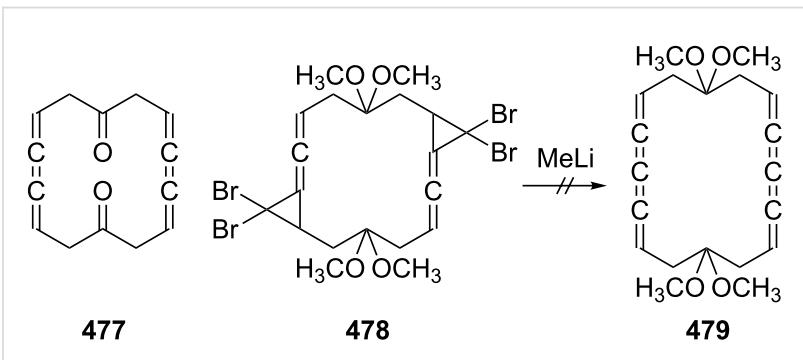

Scheme 107: The preparation of cyclic biscumulenic ring systems.

We conclude this section on cyclic bisallenes by making reference to two functionalized systems of this general type and their use for the preparation of large ring systems. In Scheme 108 it is shown how a compound of type $\mathbf{3 2 9}$ (Scheme 76) was used by Williams and co-workers to develop the synthetic methodology for the efficient preparation of various compounds related to erythronolide A $[190,256]$. Model system 480 was first oxidized with dimethyldioxirane (DMDO), and the resulting bis(spiro)diepoxide derivative $\mathbf{4 8 1}$ was subsequently opened by

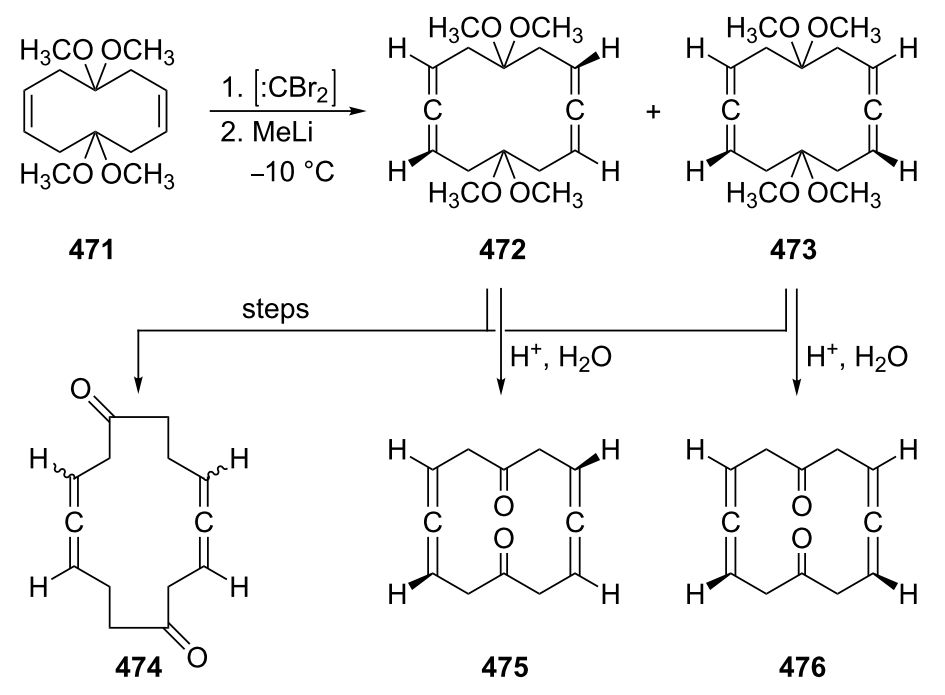

Scheme 106: The preparation of keto derivatives of cyclic bisallenes. 
<smiles>CCCC=C(C)COC(=O)CCC=C=C(C)O[Na]</smiles>

480

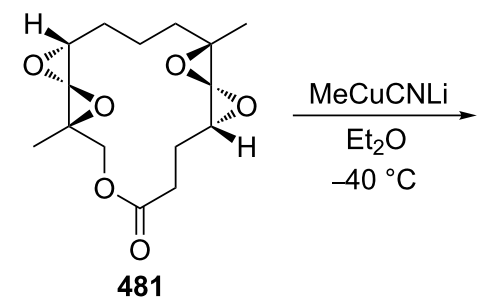<smiles>C[C@H]1CCCC(=O)OC[C@](C)(O)C(=O)[C@H](C)CC[C@@](C)(O)C1=O</smiles>

$482(22 \%)$

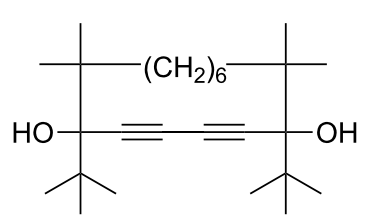

483<smiles>CC(C)(C)C(=C=C(Br)C(Br)=C=C(C(C)(C)C)C(C)(C)C)C(C)(C)C</smiles>

484

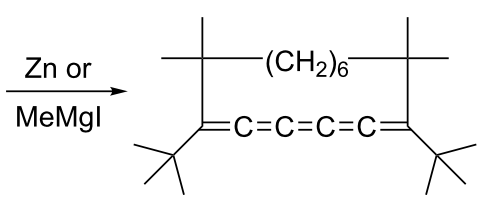

485

Scheme 108: Cyclic bisallenes in natural- and non-natural-product chemistry.

a nucleophile (a cuprate in this case) to $\mathbf{4 8 2}$. Although the yield of $22 \%$ is not high, it is sufficient considering what has been accomplished from the view of structural complexity. The second example involves the macrocycle 484. For its preparation the bispropargylic alcohol $\mathbf{4 8 3}$ (itself obtained from the appropriate precursor by a Glaser coupling procedure) is treated with $\mathrm{PBr}_{3}$ (Scheme 108) [60]. On reduction of 484, which itself is one of the rare 3,4-dihalo-diallenes (see above, Section 1.2, Scheme 15) with zinc powder, the [5]cumulene $\mathbf{4 8 5}$ is produced. Other bridging elements than the shown polymethylene chain may be introduced to provide comparable ring systems in corresponding yields. The structure of $\mathbf{4 8 4}$ was established by X-ray structural analysis with the two bromine substituents in a transoid arrangement.

Cyclic bisallenes belong to the few compounds of this class of unsaturated systems for which metal complexes have been reported. Thus both 1,2,6,7-cyclodecatetraene $(n=2,453)$ and $1,2,9,10$-cyclohexadecatetraene $(n=5,455)$ on heating with $\mathrm{Fe}_{3}(\mathrm{CO})_{12}$ in boiling hexane yield red-orange bicyclic oligomethylene ethane diiron hexacarbonyl complexes. The structures of $\mathbf{4 8 6}$ and $\mathbf{4 8 7}$ have been derived from spectroscopic data and X-ray structural analyses (Scheme 109) [257]. Furthermore, $\mathbf{4 5 3}$ has been shown to form well-defined crystalline $\pi$-complexes with silver(I) nitrate and copper(I) chloride. According to spectroscopic and analytical data these metal complexes are of polymeric nature and possess structures $\mathbf{4 8 8}$ and 489 (Scheme 109) [258].

\subsubsection{Exocyclic bisallenes}

Bisallenes in which the two allene groups are outside of an alicyclic ring system (i.e., exocyclic) have hardly been described in the chemical literature. Usually the ring systems in

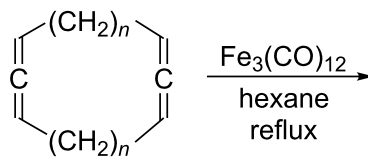

$453(n=2)$

$455(n=5)$

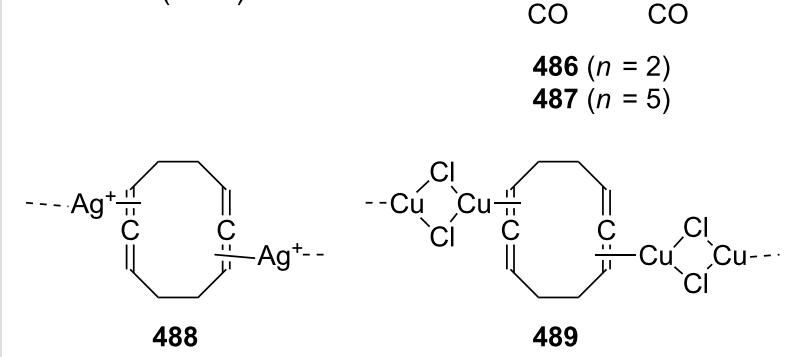

Scheme 109: The preparation of iron carbonyl complexes from cyclic bisallenes.

these derivatives are of aromatic nature (see Section 4.1). Many such systems with interesting chemical properties can be imagined; Figure 6 displays just three of them, the hydrocarbons 490-492. Any of these (and many more) should display interesting chemical behavior in, e.g., isomerization and addition reactions.<smiles>C=C=CC1(C=C=C)CC1</smiles>

490

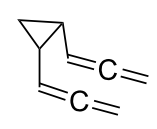

491

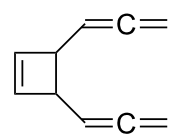

492
Figure 6: A selection of unknown exocyclic bisallenes that should have interesting chemical properties. 


\section{Bisallenes as reactive intermediates}

We have already mentioned several bisallenes as reactive intermediates that easily undergo cycloadditions and/or rearrangement reactions, see for example Scheme 55 (229), Scheme 79 (341) and Scheme 97 (421). Nevertheless we would like to return to this topic in a separate chapter since bisallenes have been suggested as reactive intermediates in some very interesting (multistep) transformations. In none of these examples could the bisallenic intermediates actually be isolated or directly observed.

In their studies on the thermal isomerization of cis- and trans1,2-diethynylcyclopropanes 493, Bergman and co-workers postulated the generation of 1,2,4,5-cycloheptatetraenes 494, as reaction intermediates, produced from 493 by a Cope-type isomerization (see Section 1.4.1). These highly strained cyclic bisallenes, which according to quantum chemical calculations prefer the $d, l$-configuration, immediately cycloisomerize to the bicyclo[3.2.0]heptatrienes 495 (Scheme 110) [259-261]. The next higher homologue of 493, cis- and trans-1,2-diethynylcyclobutane (496, $\mathrm{R}=\mathrm{H}$ ) was studied by Eisenhuth and Hopf $[262,263]$. Although in this case the initially generated, highly strained intermediate 497 still cyclizes to the bismethylenecyclobutene product, 499 , its by far preferred mode of stabilization involves a 1,5-bridging step and insertion of the thus produced carbene intermediate into the neighboring $\mathrm{C}, \mathrm{H}-$ bond to produce 1,2-dihydropentalene 498, in high yield. If the $\alpha$-position is blocked by a methyl substituent, $496\left(\mathrm{R}=\mathrm{CH}_{3}\right)$, the thermal isomerization of the bisacetylenic substrate initially provides the expected cyclic bisallene 500, but then takes a different course, furnishing, among other things, the indene derivatives $\mathbf{5 0 1}$ and $\mathbf{5 0 2}$.

The dehydro analogue of 497, 1,2,4,6,7-cyclooctapentaene (504, $\left.\mathrm{R}^{1}=\mathrm{R}^{2}=\mathrm{H}\right)$ was suggested by Sondheimer and Mitchell to be produced from 3,5-octadien-1,7-diyne $\left(\mathbf{5 0 3}, \mathrm{R}^{1}=\mathrm{R}^{2}=\mathrm{H}\right)$ at room temperature (Scheme 111) [264]. The monocyclic bisallene isomerizes immediately to the benzocyclobutadiene $\mathbf{5 0 5}$, which produces a stable dimer, the hydrocarbon $\mathbf{5 0 6}$, in good yield. Derivatives of $\mathbf{5 0 3}$ with, e.g., cycloalkyl and phenyl substituents behave analogously up to the benzocyclobutadiene $\mathbf{5 0 5}$ but subsequently dimerize to cyclooctatetraene $\mathbf{5 0 7}$ via an intermediate [3]ladderane [265].

In an important experiment Dehmlow and Ezimora prepared the dibromocarbene bis-adduct of cyclooctatetraene $\mathbf{5 0 8}$ and treated it with methyllithium in diethylether at $-78^{\circ} \mathrm{C}$. Rather than the expected 1,2,4,6,7,9-cyclodecahexaene (509), they isolated naphthalene (510) as the sole reaction product (Scheme 112) $[238,239]$.

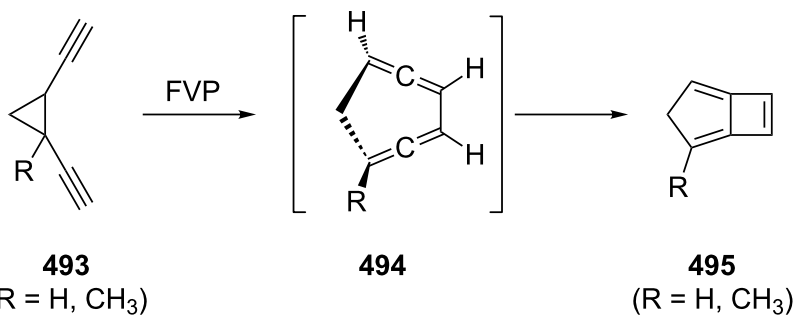

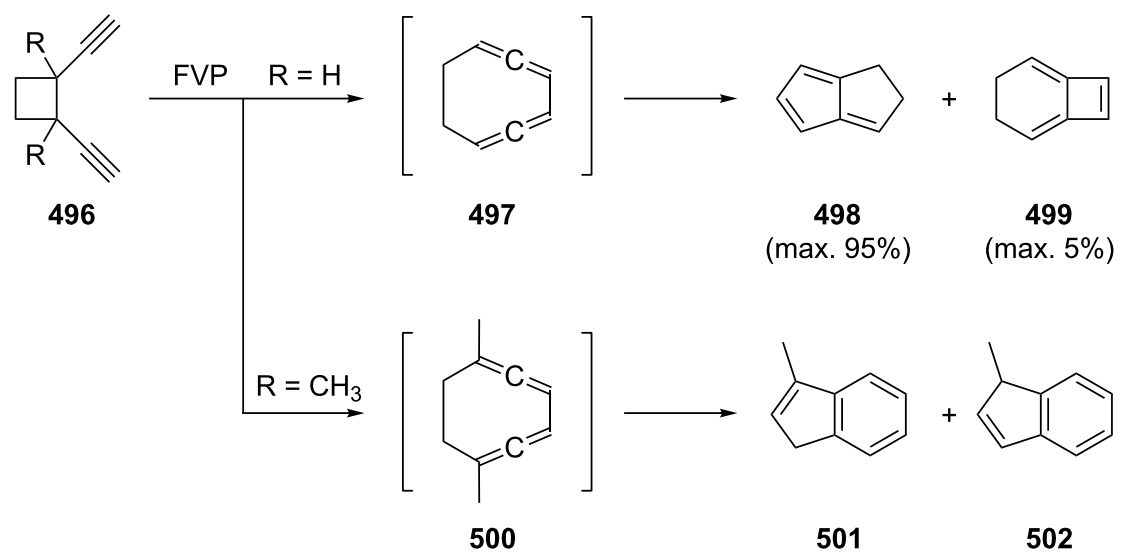


<smiles>[R]/C(C#CF)=C/C#C</smiles>

503<smiles>[R]C1=CC=CC=C([R])C([R1])=C1</smiles>

504<smiles>[R]C1=Cc2ccc([R7])c([R])c2C1=[R]=[R]</smiles>

505<smiles>[R]C1=CC2c3ccc([R])c([R])c3C2c2ccc([R])c([R])c21</smiles>

506

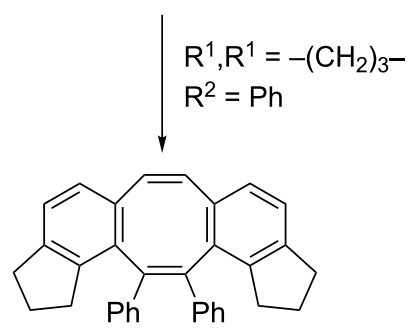

507

Scheme 111: Intermediate generation of a cyclooctapentaene.<smiles>BrC12C=CC3C1C=CC1C3(Br)C12Br</smiles>

508

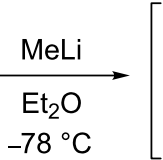

$-78^{\circ} \mathrm{C}$<smiles>C1=CC=CC=C=CC=CC=1</smiles>

509<smiles>c1ccc2ccccc2c1</smiles>

510

Scheme 112: Attempted preparation of a cyclodecahexaene.

In another thermal isomerization experiment, Vollhardt and Dower studied the conversion of 1,5,9-cyclododecatriyne (511), into [6]radialene (514), a process that in principle could take place via an intramolecular $[2+2+2]$ cycloaddition to a triscyclobutenobenzene intermediate and ring opening of the latter, or by three consecutive [3.3] sigmatropic shifts involving two semicyclic bisallene intermediates, 512 and $\mathbf{5 1 3}$ (Scheme 113) $[266,267]$. That this second alternative is indeed the more likely one was demonstrated by introducing a ${ }^{13} \mathrm{C}$-label into the sub- strate 511 and then following its distribution in the final rearrangement product $\mathbf{5 1 4}$.

A solid-state thermal transformation probably involving a functionalized derivative of a conjugated bisallene 516 was described by Tanaka and co-workers (Scheme 114) [268]. Heating the colorless propargylallene derivative 515 at ca. $200{ }^{\circ} \mathrm{C}$ causes a color change to copper brown without melting of the substrate crystals. Spectroscopic and X-ray evidence<smiles>C=C=C1CCC(=C=C=C2CCC(=C=C)C(=C=C)CC#CCCC2=C=C)C(=C)C1=C</smiles>

Scheme 113: The thermal isomerization of 1,5,9-cyclododecatriyne (511) into [6]radialene (514). 
revealed that the furofurane derivative $\mathbf{5 1 7}$ had been produced; a plausible intermediate in this interconversion is the bisallene 516, which cyclizes as indicated in Scheme 114.

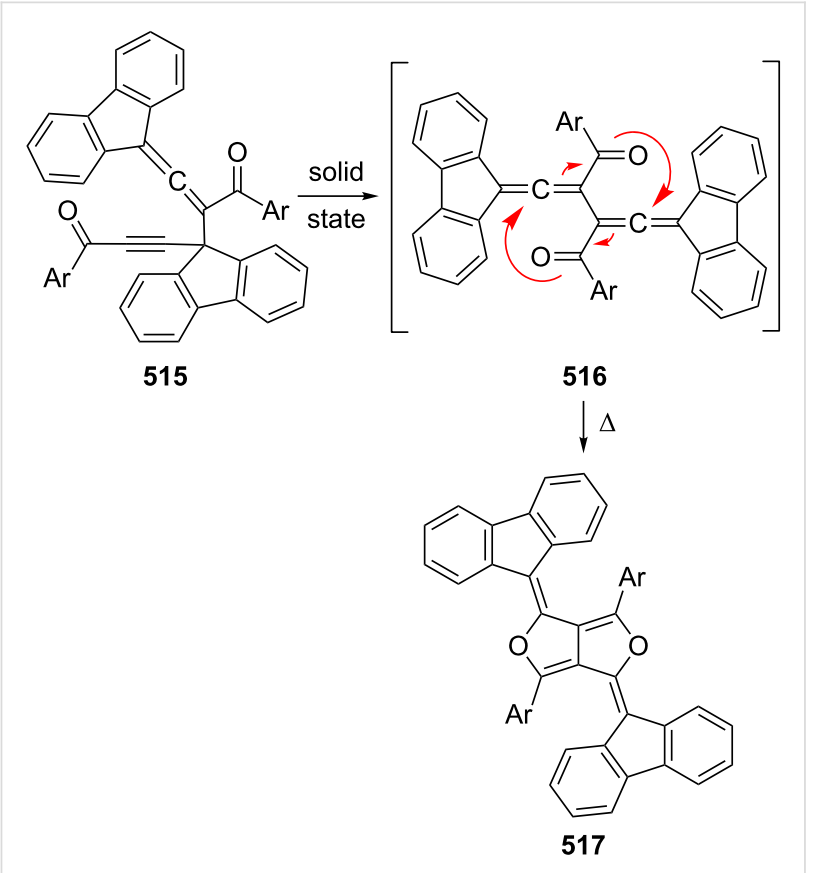

Scheme 114: An isomerization involving a diketone derived from a conjugated bisallene.

\section{Bisallenes with heteroatoms in their tethers (heteroorganic bisallenes)}

Bisallenes in which the structural element connecting the two allene moieties is or contains a non-carbon atom (usually $\mathrm{S}, \mathrm{N}$ and $\mathrm{O}$ ), form an important and interesting subgroup of this class of organic compounds. Trying to distill a unifying concept out of the vast literature is difficult. One solution may be to group the many results into two categories (Scheme 115).

In the first category we find those reactions of the heterorganic bisallenes $\mathbf{5 1 9}$ that are initiated by a (usually thermal) cycloaddition yielding a diradical intermediate, 518. Depending on the substituents present in the substrate, the actual reaction conditions and whether, for example, the reaction mixture contains other reaction partners (trapping agents), $\mathbf{5 1 8}$ can subsequently lead to quite diverse products.
In the second category we find transition-metal-initiated or catalyzed processes, which lead to cyclization products often containing medium-sized ring systems, as symbolized by $\mathbf{5 2 0}$. This reaction mode is particularly characteristic for the 1,5-diallenes already briefly discussed in Section 2.4 for all-carbon systems.

\subsection{Thermally induced reactions of heteroorganic bisallenes}

The generation and chemical behavior of many heterorganic acyclic bisallenes is summarized in Scheme 116 and basically consists of three steps [269]. In the first step the bisallene $\mathbf{5 2 2}$ is generated from a usually readily available diacetylene $\mathbf{5 2 1}$ by base treatment. This reactive species subsequently undergoes $\mathrm{C}-\mathrm{C}$ bridging to furnish the resonance-stabilized diradical $\mathbf{5 2 3} \leftrightarrow \mathbf{5 2 4}$. In the terminating step, dimerization to compounds of type $\mathbf{5 2 5}$ often occurs. In practice, the first step can be technically quite difficult, though, requiring often carefully controlled reaction conditions, which, additionally, may differ significantly from one substrate system (521) to the other. Quite often the bisallene intermediate is not detected at all, which for the present review would have meant to deal with it in Section 5.

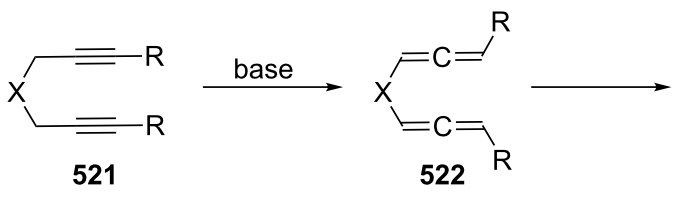

$\mathrm{X}=\mathrm{O}, \mathrm{S}, \mathrm{Se}, \mathrm{SO}, \mathrm{SO}_{2}$

$\mathrm{R}=\mathrm{H}, \mathrm{Me}, t-\mathrm{Bu}, \mathrm{iPr}, \mathrm{Ph}$

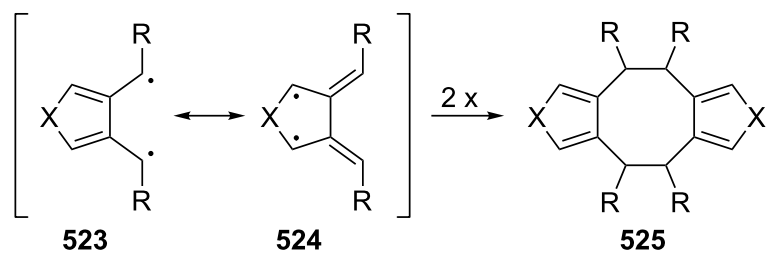

Scheme 116: Generation and thermal behavior of acyclic heteroorganic bisallenes.

A typical example, studied by Garratt and co-workers, involves the thioether $\mathbf{5 2 1}(\mathrm{X}=\mathrm{S}, \mathrm{R}=\mathrm{H})$ [270,271]. When this diacetylene was treated with $t$-BuOK in THF at $-70{ }^{\circ} \mathrm{C}$ for $40 \mathrm{~s} \mathrm{a} \mathrm{mix-}$

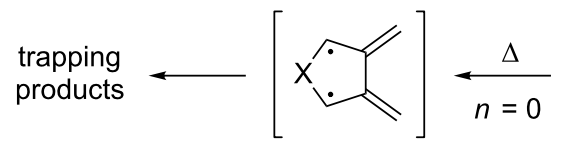

518

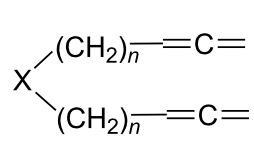

519

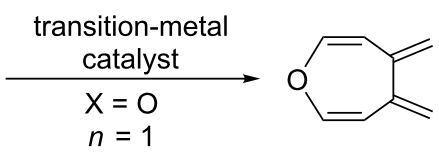

520

Scheme 115: Typical reaction modes of heteroorganic bisallenes. 
ture of $\mathbf{5 2 6}$ (93\%), the dimer $\mathbf{5 2 7}(4 \%)$ and the substrate $\mathbf{5 2 1}$ (4\%) resulted (Scheme 117) [270]. However, for the analogous ether $521(\mathrm{X}=\mathrm{O}, \mathrm{R}=\mathrm{H})$ harsher reaction conditions were required: the temperature had to be increased to $0{ }^{\circ} \mathrm{C}$ and the reaction time extended to $25 \mathrm{~min}$ [270,271]. Not surprisingly, when this process was carried out with the thioether in the presence of oxygen as a trapping reagent, the corresponding endoperoxide was isolated. The two diradical intermediates $\mathbf{5 2 9}$ and $\mathbf{5 3 0}$ can also be studied directly by stabilizing them in a matrix at low temperatures $(77 \mathrm{~K})$ as demonstrated by Berson and co-workers (Scheme 117) [272,273]. As was shown by these workers using cross-polarization magic-angle spinning (CPMAS) ${ }^{13} \mathrm{C}$ NMR spectroscopy, the reaction intermediates possess the diradical structures $\mathbf{5 2 9}$ and $\mathbf{5 3 0}$ and not, e.g., those of a bicyclic full-valence isomer. Both diradicals were generated by matrix photolysis of the precursor bisallenes $\mathbf{5 3 1}$ and by low-temperature photolysis of the corresponding diazenes $\mathbf{5 2 8}$ in these investigations $(\mathrm{X}=\mathrm{O}, \mathrm{S})$.

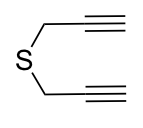

521 $(X=S ; R=H)$
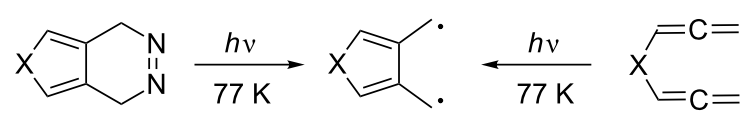

528

$(X=0, S)$

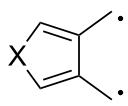

$526(93 \%)$

$529(X=S)$

$530(X=0)$

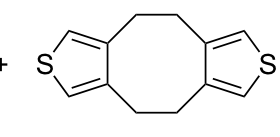

$527(4 \%)$
531 $(X=0, S)$
Scheme 117: Generation of bis(propadienyl)thioether.

Modification of the substitution pattern and/or the heteroorganic bridging element leads to substantial changes in the reaction mechanisms and the preparative outcome of these processes. For example, in a classic paper Braverman and co-workers prepared the diallenic sulfone $\mathbf{5 3 5}$ from 2-methyl-3butyne-2-ol (532) and sulfur dichloride by a double [2.3] sigmatropic rearrangement involving $\mathbf{5 3 3}$ and $\mathbf{5 3 4}$ as reaction intermediates (Scheme 118) [274,275]. On heating of $\mathbf{5 3 5}$ it undergoes quantitative cyclization to the thiophen-1,1-dioxide 537. Analogues of $\mathbf{5 3 5}$ in which the sulfone group has been substituted by $\mathrm{S}, \mathrm{Se}$ and $\mathrm{O}$ could also be prepared and they undergo the same cycloisomerization, although under milder conditions [276,277]. A mechanistic study of the rearrangement was performed by using deuterated methyl groups in e.g. 535 and studying the label distribution in the products and the isotope effect. From its absence it was concluded that the reaction takes place in two steps, with the first rate-determining step involving ring closure to an intermediate for which structure 536 can be postulated, in analogy to the above studies [274,278].

The diallenyl sulfone $\mathbf{5 3 5}$ is an interesting substrate in other respects, too. On adding bromine to it at room temperature the compound undergoes an addition reaction accompanied by a fragmentation (Scheme 119) [279]. The initially produced carbocation 538 fragments to $\mathbf{5 3 9}$, one of the isolated products, and evidently to the resonance-stabilized cation $\mathbf{5 4 0} \leftrightarrow \mathbf{5 4 1}$, since the bromine trapping products resulting from this species, the tribromides $\mathbf{5 4 2}$ and $\mathbf{5 4 3}$, can also be isolated.

On metalation of $\mathbf{5 3 5}$ with $n$-butyllithium in THF at $0{ }^{\circ} \mathrm{C}$, followed by hydrolysis/deuterolysis a remarkable dimer 545 can be isolated, the adamantanoid structure of which was established by X-ray structural analysis (Scheme 120) [280]. It has been proposed that the monoanion derived from 535 attacks a second molecule of the substrate in the "crossed" fashion shown in 544. The resulting dimer should possess a deuterium atom in the position shown in the scheme after $\mathrm{D}_{2} \mathrm{O}$ workup, and this is indeed found experimentally.

Taking diyne $\mathbf{5 2 1}$ (see Scheme 116) as the parent system, this can be varied in countless ways, for example by changing the
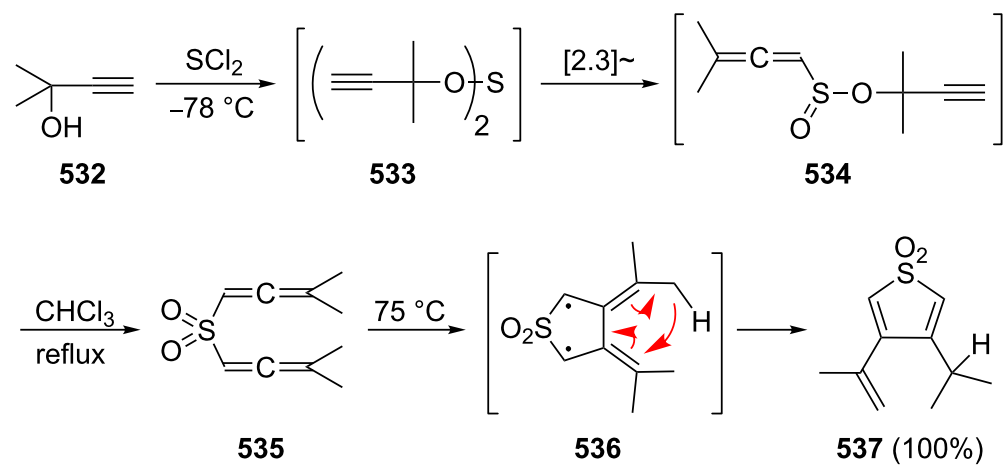
<smiles>CC(C)=C=CS(=O)(=O)C=C=C(C)C</smiles>

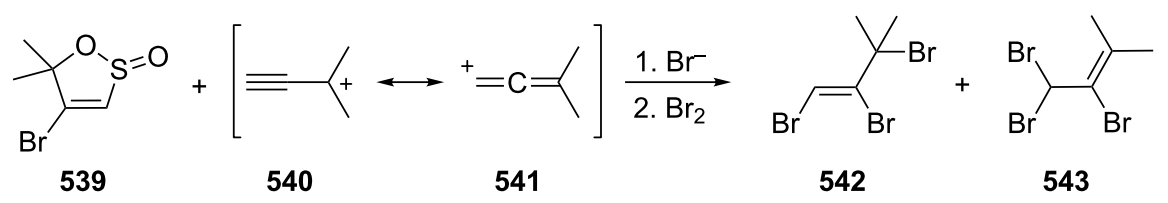

Scheme 119: Bromination of the bisallenic sulfone 535 .<smiles>CC(C)=CCCCCC(C)C</smiles>

535

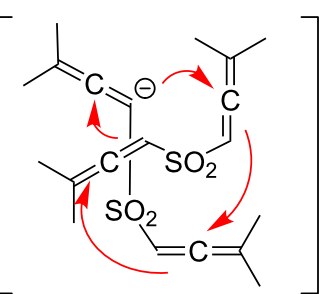

544

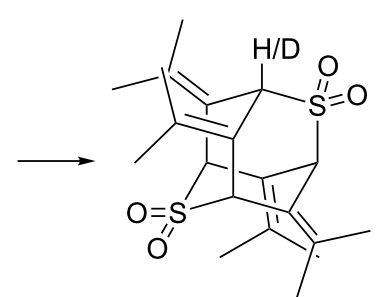

545

Scheme 120: Metalation/hydrolysis of the bisallenic sulfone 535

substituents $\mathrm{R}$ or by replacing the heteroorganic molecular "bridge" by other combinations of heteroatoms. The corresponding studies have been carried out over a vast range of exchanges/permutations, notably by Braverman and by Garratt and their co-workers. Beginning with the simple phenyl and diphenyl derivatives 546, Iwai and Ide [281] and later Garratt and Neoh [282] have shown that they isomerize in good yield $(40-100 \%)$ by base treatment to the naphthalene derivatives 551. Although several mechanisms have been proposed for this isomerization the one summarized in Scheme 121 seems to be the most likely one. The process begins with the formation of the hetero bisallene 547, which next undergoes the expected ring closure to 548. This diradical intermediate, however, carries at least one phenyl substituent, which can participate in the next step, cyclization to 549. As an "isotoluene" 549 is unstable and isomerizes to the bridged heterocycle 550. This compound, which can be isolated, finally stabilizes itself by forming the more stable naphthalene isomer $\mathbf{5 5 1}$.

An analogous mechanism can also be proposed for the isomerization of the vinyl-substituted bispropargyl ether 552, which has been studied by Ollis et al. (Scheme 122) [283-285]. In this

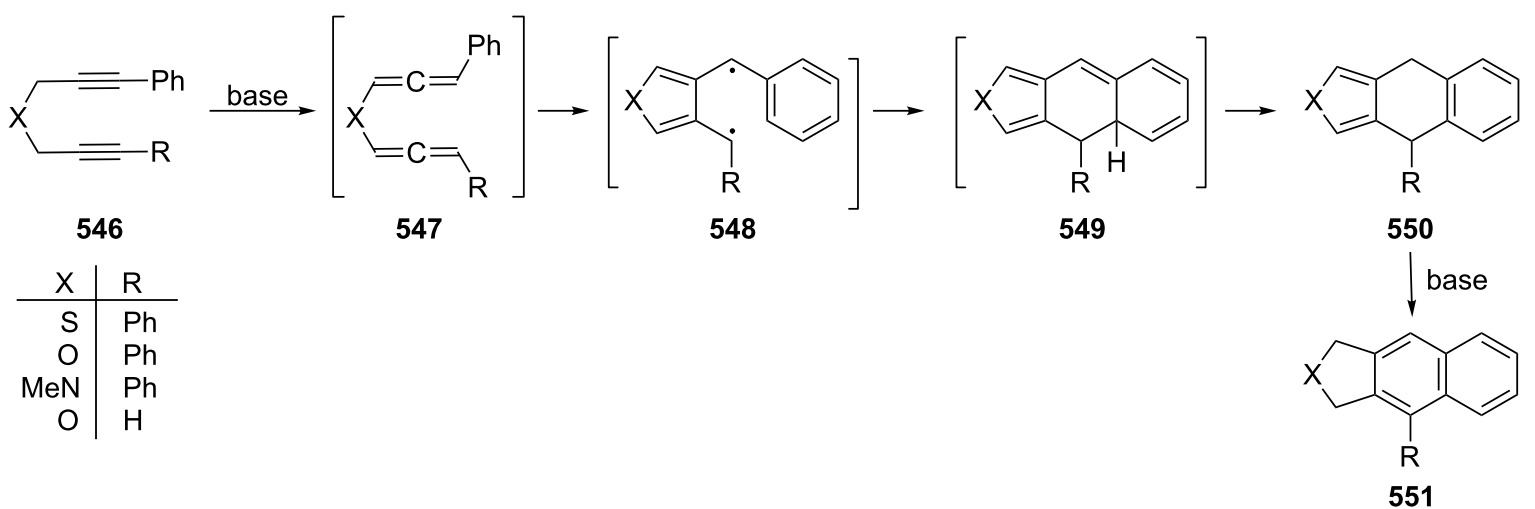

Scheme 121: Aromatic compounds from hetero bisallenes. 


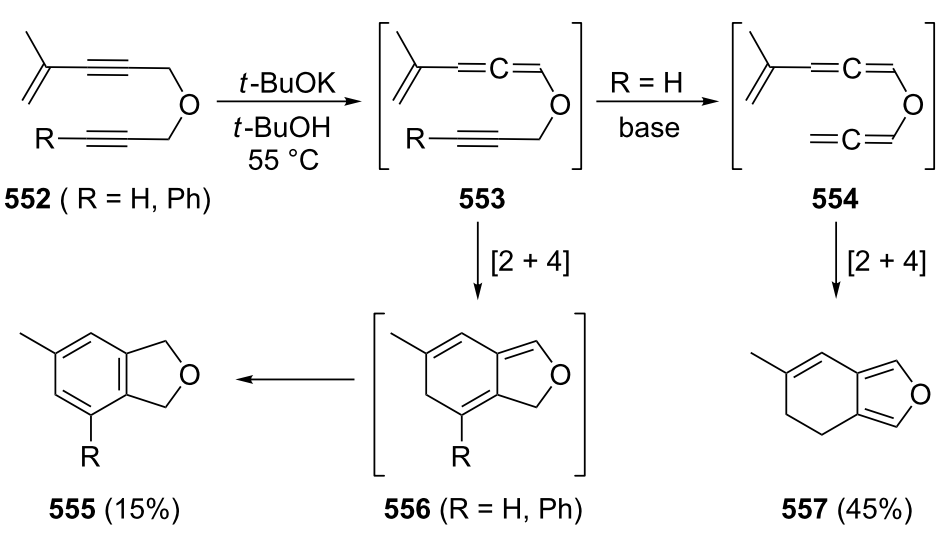

Scheme 122: Isomerization/cyclization of bispropargylic ethers.

case the initial isomerization leads to the monoallenes $\mathbf{5 5 3}$, which can either undergo a second acetylene-to-allene rearrangement and provide the bisallenyl ether $\mathbf{5 5 4}$ or participate in a Diels-Alder addition to 556. Ring closure of $\mathbf{5 5 4}$ as above leads to the furan derivative $\mathbf{5 5 7}$, whereas $\mathbf{5 5 6}$ stabilizes itself to $\mathbf{5 5 5}$.

Braverman et al. generalized these processes and, in particular, extended them to bispropargylic sulfides, selenides, sulfoxides and sulfones, which in the presence of amine bases, such as DBU, undergo facile isomerization to the corresponding bisallenes (which are usually not isolated or even detected by, e.g., NMR spectroscopy) [286-289]. The ensuing tandem cyclization and aromatization reactions are comparable to the ones discussed above. The comprehensive studies of this group can be presented in general form by the conversion of $\mathbf{5 5 8}$ (substrate) into 559, the product formed by the sequential process discussed above (Scheme 123). Some of the diradicals formed in these processes, such as $\mathbf{5 6 0}$, possess a high potential for DNA cleavage [286].

Cyclic variants of these rearrangements have been observed as early as 1964 when Eglinton, Raphael and co-workers isomerized 1,6-dithia-3,8-cyclodecadiyne (561) to the bridged thiophene 563. Presumably, 562 is generated as an intermediate in this transformation and subsequently cyclizes via the corresponding diradical intermediate followed by a hydrogen shift (Scheme 124) [290].

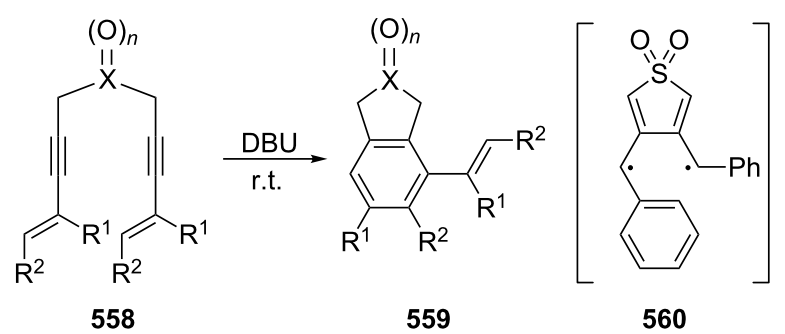

$(n=0,1,2 ; \mathrm{X}=\mathrm{S}, \mathrm{Se}$

$\mathrm{R}^{1}, \mathrm{R}^{2}=(\mathrm{CH}=\mathrm{CH})_{2}$;

$\mathrm{R}^{1}=\mathrm{CH}_{3}, \mathrm{R}^{2}=\mathrm{H}$;

$\left.\mathrm{R}^{1}, \mathrm{R}^{2}=\left(\mathrm{CH}_{2} \mathrm{CH}_{2}\right)_{2}\right)$

Scheme 123: The preparation of novel aromatic systems by basecatalyzed isomerization of bispropargyl ethers.

Modern variants involve more complex sulfides such as 564 as well as various selenides; these derivatives have been investigated by the Braverman group (Scheme 125) [291]. The formation of the product 567 can be explained readily by postulating the intermediates 565 and $\mathbf{5 6 6}$.

The isomerization of the hydroquinone-derived bissulfide $\mathbf{5 6 8}$ is, however, more complex (Scheme 126) [291]. Here the bisthiophene derivatives $\mathbf{5 6 9}$ and $\mathbf{5 7 0}$ are the expected products, but the cleavage product $\mathbf{5 7 1}$ is also produced $(\mathbf{5 7 1} / \mathbf{5 7 0} / \mathbf{5 6 9}=$ 1:10:6). It results by cleaving off the hydroquinone dianion, which is a good leaving group.

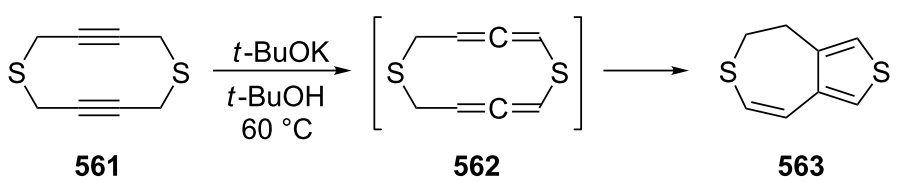

Scheme 124: The isomerization of bisacetylenic thioethers to bicyclic thiophenes. 


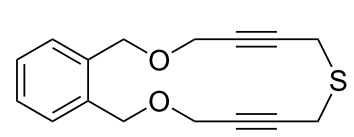

564<smiles>c1ccc(COCc2ccccc2COCc2ccsc2)cc1</smiles>

566

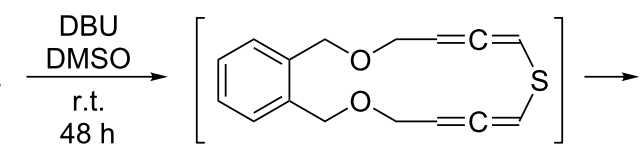

565<smiles>C1=Cc2cscc2CCOCc2ccccc2CO1</smiles>

$567(55 \%)$

Scheme 125: Aromatization of macrocyclic bispropargylic sulfides.<smiles></smiles>

568

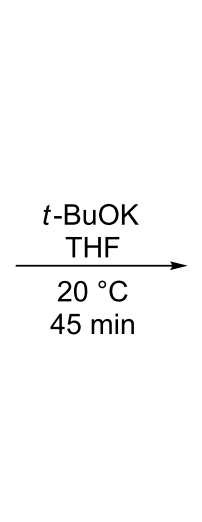

$20^{\circ} \mathrm{C}$

569<smiles>C(=C\c1cscc1CCOc1ccc(OCCc2cscc2/C=C/Oc2ccc(OCCc3ccsc3)cc2)cc1)\c1ccccc1</smiles><smiles>C(=C/c1cscc1CCOc1ccc(OCCc2cscc2/C=C/c2ccccc2)cc1)\Oc1ccc(OCCc2ccsc2)cc1</smiles>

570<smiles>C=CC#CSC#CC=C</smiles>

571

Scheme 126: Preparation of ansa-compounds from macrocyclic bispropargyl thioethers.

The discussed diradical pathway is not the only route by which a dipropargyl (or a tetrapropargyl) sulfide (disulfide) or the corresponding selenide can react (Scheme 127) [292]. As displayed in Scheme 127 the heterobisallene $\mathbf{5 7 2}$ can react according to the "normal" path via diradical 573 to the diradical cycloaromatization products as discussed above several times. Alternatively, however, the base could abstract a (nonterminal) allene proton and generate the bisallenyl anion $\mathbf{5 7 4}$ as

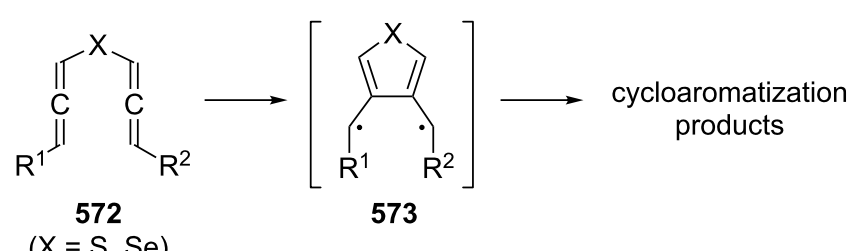

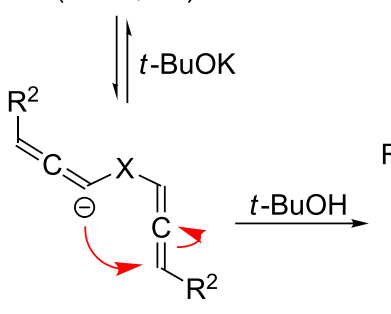

574<smiles>[R1]C=C=C1[X]C=CC([R])C1[R19]([H])([H])C</smiles>

575<smiles>[R1]C=Cc1[X]ccc1[R]</smiles>

576

Scheme 127: Alternate route for cyclization of a heterorganic bisallene. 
shown. If this attacks the other allene group and the resulting cycloallene carbanion is protonated by the solvent, then the semicyclic allene $\mathbf{5 7 5}$ results, which stabilizes itself to a vinyl thiophene/vinyl selenophene $\mathbf{5 7 6}$ under the influence of the base.

One case in which such a competing reaction has been observed, concerns the "double" bispropargylic derivative $\mathbf{5 7 7}$ in Scheme 128. Here the usual diradical pathway leads to $\mathbf{5 7 9}$ via the intermediate species $\mathbf{5 7 8}$ (actually the process will most likely occur in two steps via a diradical rather than the "tetraradical" shown in 578). The anionic cyclization pathway will lead to a mixture of the diastereomers $\mathbf{5 8 0}$ and $\mathbf{5 8 1}$ [292]. Surprisingly, no $E, E$-diastereomer of $\mathbf{5 8 0} / \mathbf{5 8 1}$ was detected in the reaction mixture.<smiles>[R]C#CCC#Cc1ccc(C#CCC#CC[X])cc1</smiles>

577

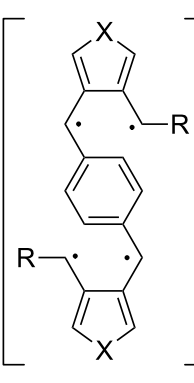

578

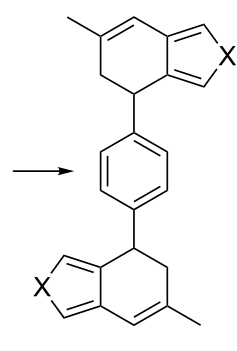

$579(36 \%)$
$\mathrm{X}=\mathrm{S} \mid t$-BuOK, THF

$\mathrm{R}=\mathrm{H} \quad 20^{\circ} \mathrm{C}, 1 \mathrm{~min}$<smiles>C(=C\c1cccs1)\c1ccc(/C=C/c2cccs2)cc1</smiles><smiles>C(=C\c1cccs1)\c1ccc(/C=C\c2cccs2)cc1</smiles>

$580(14 \%)$

Scheme 128: Multiple isomerization/cyclization of "double" bispropargylic thioethers.

Changing the bridging heteroatom to more complex molecular units opens up many new reaction pathways leading to a plethora of novel products. For brevity we only present some of these results in very condensed form, as obtained again largely by the Braverman group. For many of the mechanistic details of these processes the reader is referred to the original literature. The thienothiophene derivative $\mathbf{5 8 6}$ was produced in $\mathbf{7 0 \%}$ yield when $\gamma, \gamma$-dimethylallenyl thiocyanate (582), was treated with lithium methoxide in THF at room temperature (Scheme 129) [293]. In this multistep transformation it is assumed that $\mathbf{5 8 2}$ is first cleaved to $\mathbf{5 8 3}$ and that these two components subsequently produce bis $(\gamma, \gamma$-dimethylallenyl) disulfide (584) as the decisive intermediate. The latter is then believed to undergo a consecutive [3.3] sigmatropic rearrangement to $\mathbf{5 8 5}$ followed by a double Michael addition to furnish the bicyclic product $\mathbf{5 8 6}$.

On heating, bis $(\gamma, \gamma$-dimethylallenyl) thiosulfonate (587) undergoes a series of rearrangement and cyclization reactions to provide the three thiophene derivatives 588-590. The transformation strongly depends on the nature of the solvent and the isomerization temperature. For example, in chloroform at $55{ }^{\circ} \mathrm{C}$ it yields the three products in 47:6:47 ratio in 50\% total yield after $12 \mathrm{~h}$, whereas in DMSO at $40{ }^{\circ} \mathrm{C}$ the yield increases to $80 \%$ after $12 \mathrm{~h}$ and only the first two products are obtained in $72: 28$ ratio (Scheme 130) [293,294]. Whereas $\mathbf{5 8 8}$ and $\mathbf{5 8 9}$ are presumably formed by an ionic mechanism (hence the solvent dependency of the reaction), the authors suggest a carbene-diradical sequence for the generation of the thiophene derivative $\mathbf{5 9 0}$ as summarized in Scheme 130 also. The isomerization begins with a [3.3] sigmatropic shift, which converts the substrate $\mathbf{5 8 7}$ into 591, an intermediate that by sulfur dioxide extrusion is transformed into the carbene 592. On cyclization of the latter, intermediate $\mathbf{5 9 3}$ is generated, which via its diradical resonance structure $\mathbf{5 9 4}$ is converted to the isolated product $\mathbf{5 9 0}$ by a hydrogen-transfer step.

When $\mathbf{5 8 7}$ is oxidized with meta-chloroperbenzoic acid ( $m$-CPBA) at $0{ }^{\circ} \mathrm{C}$ the bisallenic $\alpha$-disulfone $\mathbf{5 9 5}$ is produced; it is much more reactive than its saturated counterpart and undergoes a multistep rearrangement to the highly functionalized bicyclic product $\mathbf{5 9 6}$ as well as a cleavage reaction of the $\mathrm{S}-\mathrm{S}$<smiles>CC(C)=C(C)C(C)(C)C</smiles> 
<smiles>CC(C)=C=CSS(=O)(=O)C=C(C)C</smiles>

587<smiles>CC1(C)OS(=O)C(C)(C)c2cscc21</smiles>

588<smiles>CC1(C)c2cscc2C(C)(C)S1(=O)=O</smiles>

589<smiles>C=C(C)c1cscc1C(C)C</smiles>

590

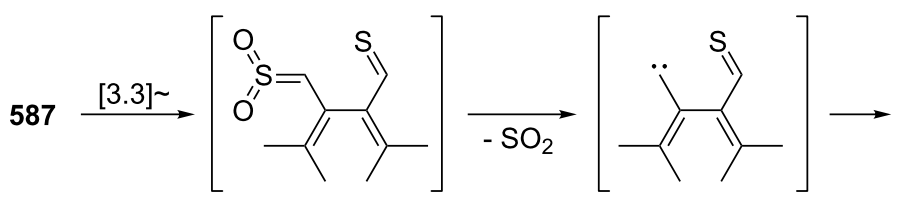

591

592

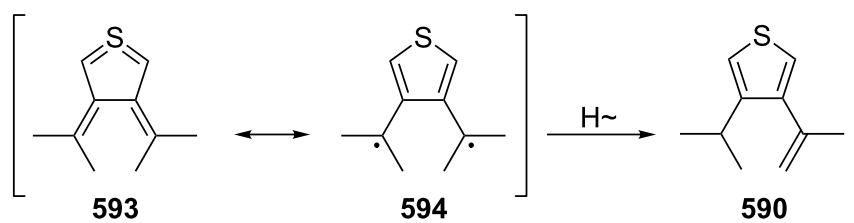

Scheme 130: Thermal cyclization of a bisallenyl thiosulfonate.

bond, which ultimately leads to the monocyclic product $\mathbf{5 9 7}$ (Scheme 131) [295]. The reduced form of $\mathbf{5 9 5}$, i.e. intermediate 599, can be accessed by a double [2.3] sigmatropic rearrangement of the dipropargylic disulfide 598. This heteroorganic bisallene subsequently cyclizes to the bicyclic product $\mathbf{6 0 0}$, among other things, in a multistep tandem process of high atom economy [296,297]. Note the structural similarity of product $\mathbf{5 8 6}$ in Scheme 129 with that of $\mathbf{6 0 0}$ in Scheme 131.

Bisallenes containing nitrogen atoms in their spacers, i.e., bisallenic amines, have been obtained by various routes. Thus, Miginiac and co-workers prepared the bisallene $\mathbf{6 0 2}$ by an aminomethylation/desilylation process in which the propargylic substrate 601 is converted into the tertiary amine $\mathbf{6 0 2}$ (Scheme 132) [298,299]. The method reported by Sato [229] and by Hara et al. [231], mentioned already for all-carbon systems above (see Schemes 101 and 103), has also been employed for the preparation of heterocyclic semicyclic bisallenes, as demonstrated by the conversion of $\mathbf{6 0 3}$ into 604 [231].

\subsection{Transition metal-induced reactions of heteroor-} ganic bisallenes

Principally, hetero bisallenes $\mathbf{6 0 5}$ can be employed in cyclization reactions in three ways (Scheme 133).

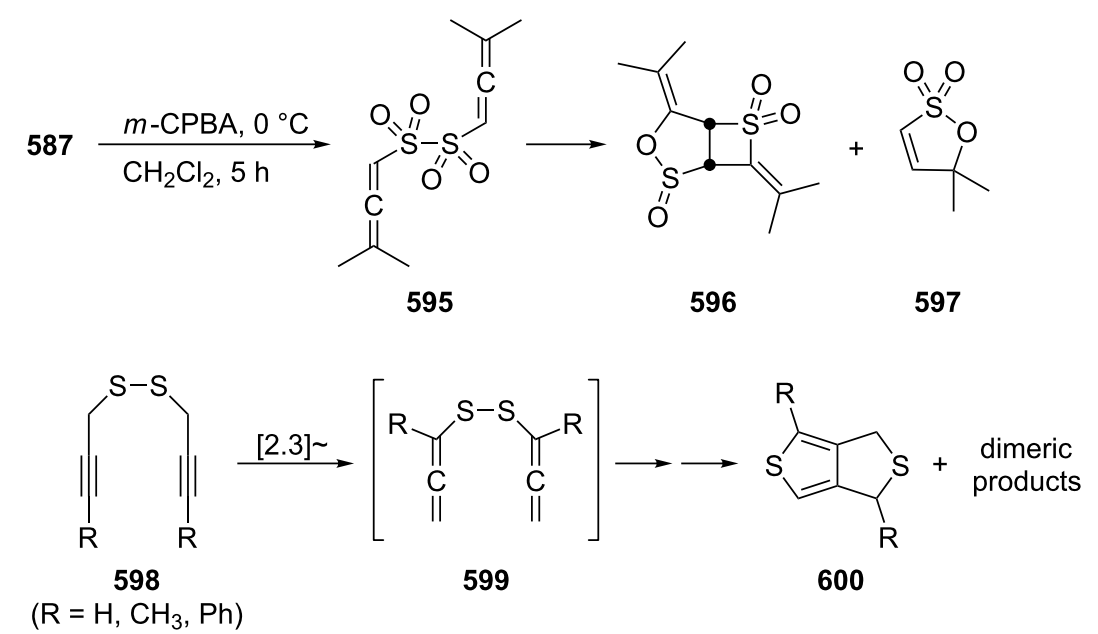




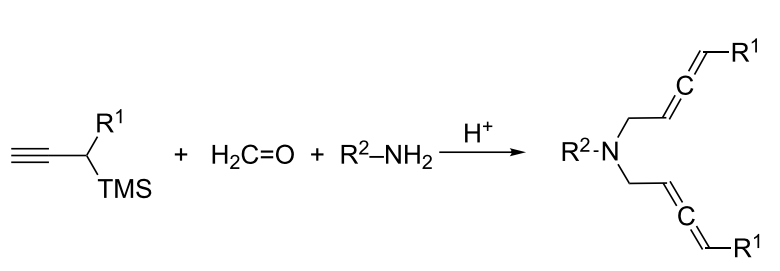

601 $\left(\mathrm{R}^{1}=\mathrm{CH}_{3} ; \mathrm{R}^{2}=n-\mathrm{Bu}: 36 \%\right)$

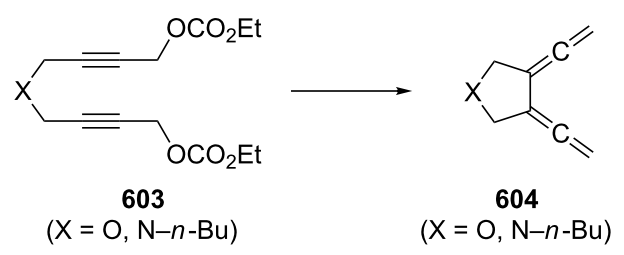

Scheme 132: Further methods for the preparation of heteroorganic bisallenes.

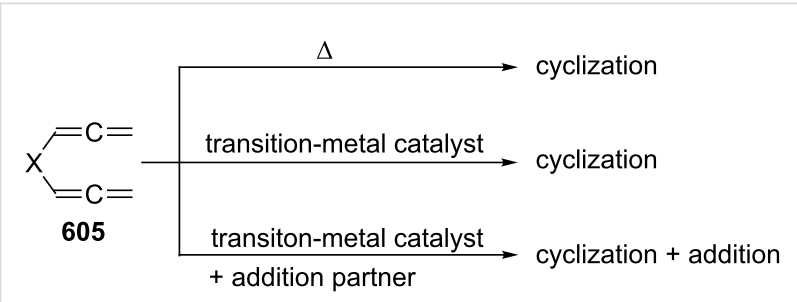

Scheme 133: Cyclization reactions of heteroorganic bisallenes.

On heating without any addition of other reagents, they could undergo a $[2+2]$ cycloaddition as described already in Section 6.1. This is, incidentally, the most characteristic reaction of allene itself. For the derivatives $\mathbf{6 0 5}$, which are connected by a spacer $-\mathrm{X}$ - and hence less symmetric than allene, the cycload- dition could take place in head-to-head or head-to-tail fashion and involve either one of the two double bonds. One would therefore expect a complex mixture of [2+2] cycloadducts [300]. The second cyclization mode, taking place in the presence of a (transition) metal catalyst, could lead to a similar set of structural isomers. However, we would expect a greater selectivity here, as in many other metal-catalyzed (cyclo)additions. In the third case, the cyclization takes place in the presence of another component in stoichiometric amounts (carbon monoxide, simple amines, but also more complex organic compounds, see below). The prediction of the stereochemical selectivity is even more difficult in this case.

As it turns out, all types of cycloadditions of the heterorganic bisallenes have been reported. A typical example of the first reaction type has been described by $\mathrm{Ma}$ and co-workers (Scheme 134) [179]. Heating the 1,5-bisallene $\mathbf{6 0 6}$ provided the bicyclo[5.2.0]system $\mathbf{6 0 7}$ in very good yields. The product resulting from the reaction of the "inner" allene double bonds, i.e. the bicyclo[3.2.0] system $\mathbf{6 0 8}$, was only produced as a minor cycloadduct (ratio 607/608 $=88.5: 11.5$ ). Obviously, both cycloadducts are head-to-head products. For the next higher homologue, the 1,6-bisallene 609, the selectivity was even better with $\mathbf{6 1 0}$ formed exclusively in nearly quantitative yield [183].

Among the processes of the second category (reactions of heterorganic $\alpha, \omega$-bisallenes in the presence of a transition-metal catalyst) the following characteristic example by Mukai and co-workers may be cited (Scheme 135) [177]. Heating of the bissulfone diallene $\mathbf{6 1 1}$ in toluene solution in the presence of [ $\left.\{\mathrm{RhCl}(\mathrm{CO}) \mathrm{dppp}\}_{2}\right]$ at $80^{\circ} \mathrm{C}$ results in cyclization to the eightmembered ring compound $\mathbf{6 1 2}$ in good yield; the process is<smiles>C=CC(=C[As])CN([Al])CC=C=C(C)C</smiles>
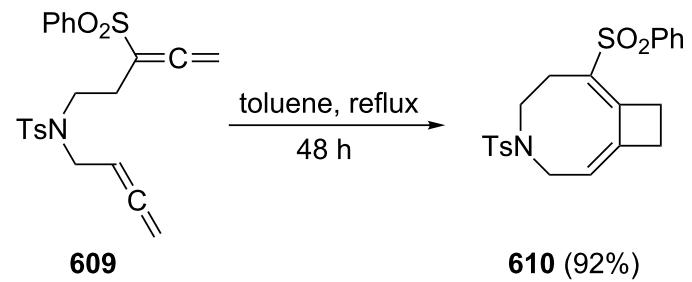
accompanied by the formation of a $[2+2]$ cycloadduct and a Pauson-Khand product (see below), both generated in small amounts $(10 \%$ each). Formally, the reaction constitutes an intramolecular ene reaction. The more likely mechanism involves the formation of a rhodacycle intermediate, which undergoes a 1,5-hydrogen shift. Reductive elimination under $\mathrm{Rh}(\mathrm{I})$ removal terminates the sequence [177]. If diketones $\mathbf{6 1 3}$, rather than the above disulfones, are employed in this process, the reaction takes a different course as shown by Ma and co-workers [301]. Now, one of the carbonyl functions is incorporated into the ring system and the bridged furane derivative $\mathbf{6 1 4}$ is produced in very good yields. Replacing the amine function in 613 by oxygen furnishes a product 614 with $\mathrm{X}=\mathrm{O}$; this is, however, accompanied by small amounts of an isomer with a semicyclic double bond in the seven-membered ring, rather than an endocyclic one [181,182].<smiles>C=C=C(CN([Al])CCC(=C=C)S(=O)(=O)c1ccccc1)c1ccccc1</smiles><smiles>C=C1C=CN([Al]C)CCC([R6](=O)(O)c2ccccc2)C1=C</smiles><smiles>[X]CC(C=C=C)C([R])=O</smiles>
$\underset{\text { acetone, } 25^{\circ} \mathrm{C}, 1 \mathrm{~h}}{\stackrel{5 \mathrm{~mol} \%\left[\mathrm{RhCl}(\mathrm{CO})_{2}\right]_{2}}{\longrightarrow}}$

$613(X=N T s ; R=E t)$ $612(71 \%)$

Scheme 135: Cyclization of a bisallenic tertiary amine in the presence of a transition-metal catalyst.

More and more reports are appearing in the chemical literature in which an additional component is added in stoichiometric amounts to the catalyst/1, $\omega$-bisallene mixture. In these cases the third component is incorporated into the resulting ring system and complex bicyclic products carrying useful functional groups are generated. A case in point is provided by various Pauson-Khand reactions starting from heteroorganic bisallenes. For example, Mukai and co-workers have shown that the bisallene ether $\mathbf{6 1 5}$ in the presence of rhodium catalysts and carbon monoxide yields the bicyclic ketone 616 in excellent yields (Scheme 136) [177]. The activating $\mathrm{PhSO}_{2}$-groups can be removed from the ring system by treatment with excess tri- $n$ butyltin hydride and higher homologues of, e.g., 616 have also been prepared. The mechanism proposed for the cyclization involves the formation of rhodacyclic intermediates.

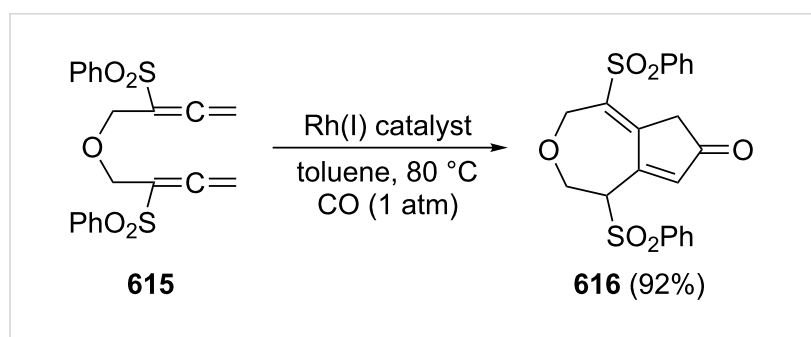

Scheme 136: A Pauson-Khand reaction of a bisallenic ether.

A sandwich-type triple cyclization of two equivalents of the 2,3-allenoic acid 617 and the bisallenic amine 618 takes place when a mixture of these components is treated with various Pd-complexes to yield the cis-configured 2:1 adducts 619 in medium to good yields $(30-80 \%)$; as a side product $\mathbf{6 2 0}$ is produced in up to $20 \%$ yield (Scheme 137) [178]. The allcarbon analogue of this case has already been discussed above (see Scheme 74).

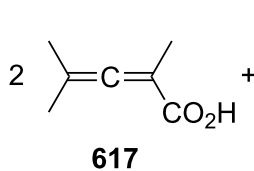<smiles>C=C=CCN([AlH2])CC=C=C</smiles>

$\left[\mathrm{PdCl}_{2}(\mathrm{PhCN})_{2}\right]$ benzoquinone $70{ }^{\circ} \mathrm{C}, 2 \mathrm{~h}$

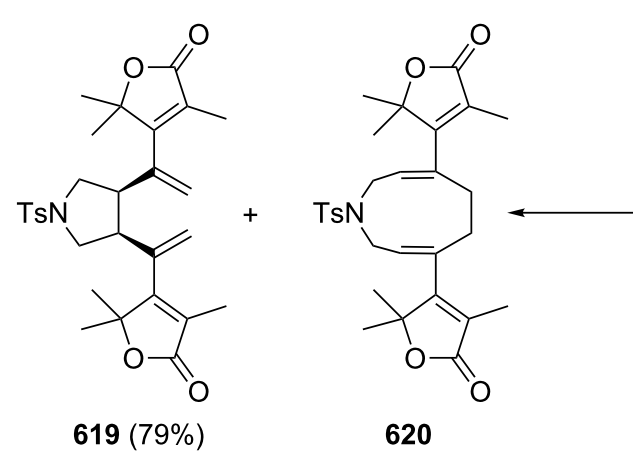

Scheme 137: Formation of a 2:1adduct from two allenic substrates.

A Pd-catalyzed carbocyclization of $\mathbf{6 1 8}$ by way of a silastannylation has been reported by Kang and co-workers (Scheme 138) $[186,187]$. Thus, when the tertiary amine $\mathbf{6 1 8}$ is heated in THF under reflux in the presence of (trimethylsilyl)tri- $n$-butylstannane and a Pd catalyst, it cyclizes in good yield to the transconfigured five-membered ring system 623. It is assumed that a $\mathrm{Me}_{3} \operatorname{SiPdSn}(n-\mathrm{Bu})_{3}$ species adds to one of the allene groups of 618 in the first step to produce a $\sigma$ - or $\pi$-allylpalladium complex 621. Because of steric hindrance this adopts another conformation, 622, which fulfills the necessary conditions to cyclize to 623. When the trimethylsilylstannane is replaced by $(n-\mathrm{Bu})_{3} \mathrm{Sn}-\mathrm{Sn}(n-\mathrm{Bu})_{3}$, a cyclization reaction occurs with $\mathbf{6 1 8}$ 


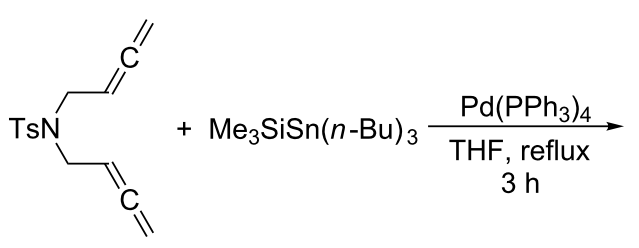

618

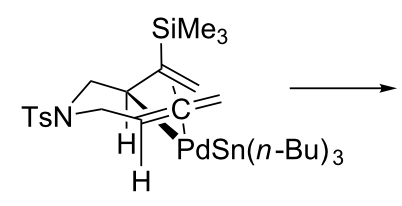

621

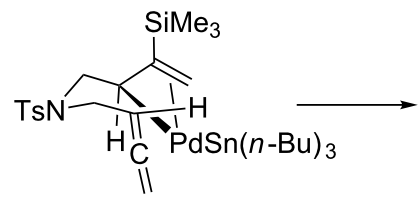

622

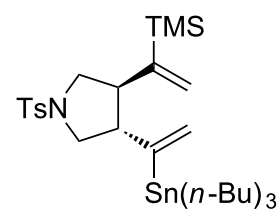

$623(78 \%)$<smiles>C=C(SCCCC)C(=C)C1CN([Te])CC1C(=C)SCC(C)C</smiles>

$624(73 \%)$

Scheme 138: A ring-forming silastannylation of a bisallenic tertiary amine.

too, but it leads to the cis-configured product 624; steric reasons are held responsible for this stereoselectivity.

That these cyclizations can be developed into three-component routes yielding, e.g., a functionalized ten-membered ring was demonstrated by Ma and co-workers (Scheme 139) [184]. Bisallenes of the general type $\mathbf{6 2 5}$ afforded the cyclodecadiene derivatives 626 in moderate to good yields (40-60\%) when reacted with organic halides and primary amines in the presence of $\operatorname{Pd}(0)$ catalysts. The products $\mathbf{6 2 6}$ are produced with high chemo- and stereoselectivity, and a mechanism involving two $\pi$-allylic palladium intermediates has been proposed to account for this result.

We close this review with still another coupling/cyclization reaction in which different propargylic carbonates, phenylboronic acids and heterorganic $\alpha, \omega$-bisallenes are reacted with

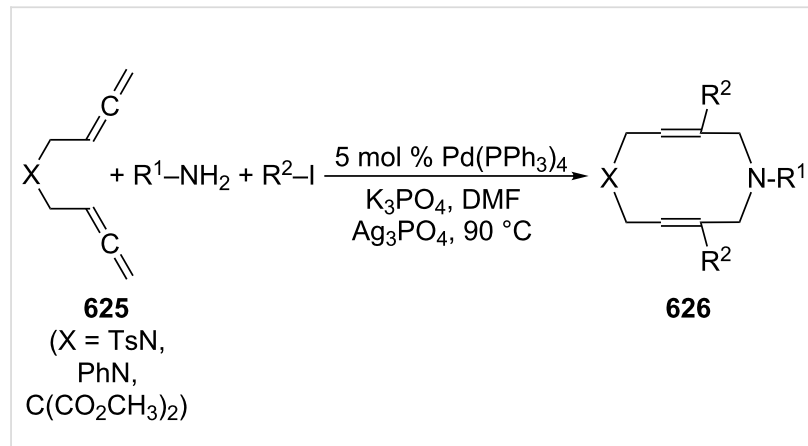

Scheme 139: A three-component cyclization involving a heterorganic bisallene.

each other in the presence of $\left[\operatorname{Pd}(\mathrm{dba})_{2}\right]$. A typical example is shown in Scheme 140 [180]. In this case the bromocarbonate $\mathbf{6 2 7}$ is coupled with 4-chlorophenylboronic acid (628) and the

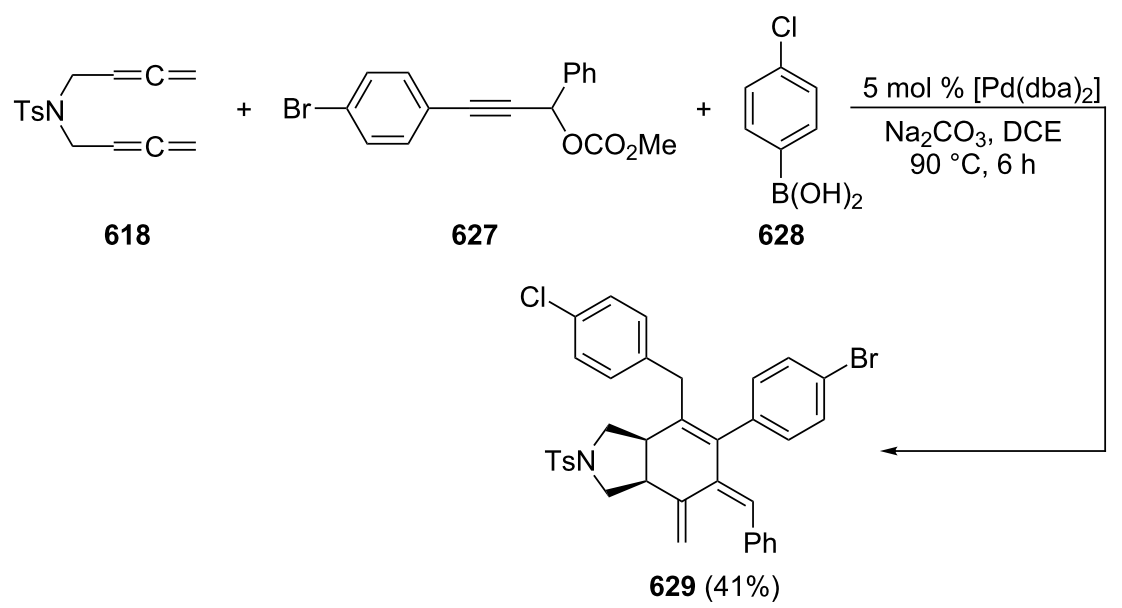

Scheme 140: Atom-economic construction of a complex organic framework from a heterorganic $\alpha, \omega$-bisallene. 
bisallene 618 to yield the adduct $\mathbf{6 2 9}$ in $41 \%$ yield. The product possesses a $c i s$-junction between its five- and six-membered rings and is produced as the single diastereomer shown. The yields of this remarkable process vary between 40 and $80 \%$.

\section{Conclusion}

Beginning with the simplest possible bisallene, 1,2,4,5-hexatetraene (2), one of the isomers of benzene, we have seen how this class of compounds has rapidly gained importance in structural and synthetic organic chemistry during the past decades. Especially the past few years have witnessed the development of many new approaches to complex organic target molecules by a relatively small number of steps. We assume that this trend will remain strong, since allenes and bisallenes, as well as other highly unsaturated $\pi$-systems, with their high concentration of $\pi$-electrons on a relatively small number of carbon atoms are very attractive substrates for the rapid and efficient generation of molecular complexity with excellent atom economy.

Needless to say, there are a few neglected areas in bisallene chemistry. One concerns the systematic study of organometallic compounds possessing more than one allene moiety, whether these are complexed bis (or tris and oligo) allenes or covalently bonded derivatives. Bis(allenyl)mercury seems to be the only organometallic compound so far in which the allene groups are bonded by covalent bonds to a metal atom [302,303]. Another neglected area concerns the photochemistry of bisallenes. The photochemistry of allenes as a whole has so far not received the attention it clearly deserves [304-307]. It is likely that novel phototransformations, preferentially photoadditions and photoisomerizations, will be discovered on closer inspection and with a larger variety of allenes than studied so far. Furthermore, the number of bisallenic natural products that have been described so far is very small. In this area one can assume that more discoveries will be made once a directed search is initiated [308]. The occasional use of bisallenes in polymer chemistry has been pointed out in this review (see Section 4.1). More results should be forthcoming in this field with compounds other than the mentioned diallenes serving as monomers.

As far as the synthesis of bisallenes is concerned we predict that the classical routes to these compounds, i.e. base-catalyzed or thermal isomerization reactions of acetylenes (the DMSapproach), will slowly fade out and will be replaced by modern, largely metal-induced coupling reactions. These reactions often take place under mild conditions, which is an important prerequisite for the preparation of highly reactive derivatives; are in many cases more specific than the classical routes; produce a smaller number of side products; and should allow the introduction of additional, preparatively useful functional groups (especially carbonyl functions).
In addition, looking at the many novel and often highly imaginative recent developments in the field of highly unsaturated compounds [309] makes one optimistic about the future of diand oligoallene chemistry.

\section{Acknowledgements}

H.H. is particularly grateful to Professor Michael S. Sherburn, Research School of Chemistry, Australian National University, Canberra, for giving him the opportunity to review this rather heterogeneous field during a stay in his research group as a visiting scientist in January and February 2012. To spend these two months in an intellectually stimulating environment was most enjoyable. That he could flee from the ice-cold Northern German winter to a warm Canberra summer was an additional important bonus. G.M. thanks the Studienstiftung des deutschen Volkes and the Fonds der Chemischen Industrie for scholarships.

\section{References}

1. Rocke, A. Image and Reality; The University of Chicago Press: Chicago, 2010; p 131.

See for the most up to date study on the problem of the early history of the determination of the benzene structure, which interests historians of science until the present day.

2. Schultz, G. Ber. Dtsch. Chem. Ges. 1890, 23, 1265-1312. doi:10.1002/cber.189002301204

3. Loschmidt, J. Chemische Studien 1861, 30, 58-59.

4. Couper, A. S. Ann. Chim. Phys. 1858, 53, 469-489.

5. Couper, A. S. Philosophical Magazine Fourth Series 1858, 16 , 104-116.

6. Hopf, H. Angew. Chem., Int. Ed. Engl. 1970, 9, 732. doi:10.1002/anie.197007321

7. Farley, E. D.; Marvel, C. S. J. Am. Chem. Soc. 1936, 58, 29-34. doi:10.1021/ja01292a008

8. Marvel, C. S.; Peppel, W. J. J. Am. Chem. Soc. 1939, 61, 895-897. doi:10.1021/ja01873a037

9. Kuhn, R.; Fischer, H. Chem. Ber. 1961, 94, 3060-3071. doi:10.1002/cber.19610941136

10. Jacobs, T. L.; Prempree, P. J. Am. Chem. Soc. 1967, 89, 6177-6182. doi:10.1021/ja01000a031

11. Pasto, D. J.; Chou, S.-K.; Waterhouse, A.; Shults, R. H.; Hennion, G. F. J. Org. Chem. 1978, 43, 1385-1388. doi:10.1021/jo00401a021

12. Krause, N.; Hashmi, A. S. K., Eds. Modern Allene Chemistry Vol. I and Vol. II; Wiley-VCH: Weinheim, 2004. doi:10.1002/9783527619573

13. Cumulenes and Allenes. In Science of Synthesis; Krause, N., Ed.; Thieme: Stuttgart, 2008; Vol. 44. Krause, N., Ed. Cumulenes and Allenes. In Science of Synthesis, Vol. 44; Thieme: Stuttgart, 2008.

14. Brummond, K. M. Beilstein J. Org. Chem. 2011, 7, 394-395. doi:10.3762/bjoc.7.50

15. Huang, W.; Fang, D.; Temple, K.; Tidwell, T. T. J. Am. Chem. Soc. 1997, 119, 2832-2838. doi:10.1021/ja963496m

16. Banert, K.; Groth, S.; Hückstädt, H.; Lehmann, J.; Schlott, J.; Vrobel, K. Synthesis 2002, 1423-1433. doi:10.1055/s-2002-33109 
17. Calejos-Gaspar, B.; Laubender, M.; Werner, H. J. Organomet. Chem. 2003, 684, 144-152. doi:10.1016/S0022-328X(03)00521-7

18. Vacher, A.; Benameur, A.; Ndiaye, C. M.; Touchard, D.; Rigaut, S. Organometallics 2009, 28, 6096-6100. doi:10.1021/om9006449

19. Rigaut, S.; Perruchon, J.; Guesmi, S.; Fare, C.; Touchard, D.; Dixneuf, P. H. Eur. J. Inorg. Chem. 2005, 447-460. doi:10.1002/ejic.200400457

20. Hopf, H. Allenic Hydrocarbons - Preparation and Use in Organic Synthesis. In Modern Allene Chemistry; Chapter 5; Krause, N.; Hashmi, A. S. K., Eds.; Wiley-VCH: Weinheim, 2004; pp 185-241. See for a discussion of these different structural combinations.

21. Hopf, H.; Böhm, I.; Kleinschroth, J. Org. Synth. 1981, 60, 41-48.

22. Hopf, H.; Lenich, F. T.; Maurer, H. unpublished results.

23. Hopf, $\mathrm{H}$. The Chemistry of Functional Groups. In The chemistry of ketenes, allenes, and related compounds; Patai, S., Ed.; John Wiley \& Sons: Chichester, 1980; Vol. II, pp 779-901. doi:10.1002/9780470771617.ch7 See for a review of allene chemistry up to ca. 1980

24. Sigman, M. S.; Eaton, B. E. J. Am. Chem. Soc. 1996, 118, 11783-11788. doi:10.1021/ja962908o

25. Drachenberg, K. J.; Hopf, H. Tetrahedron Lett. 1974, 15, 3267-3270. doi:10.1016/S0040-4039(01)91880-5

26. Skattebøfl, L. Tetrahedron 1967, 23, 1107-1117. doi:10.1016/0040-4020(67)85060-9

27. Ruitenberg, K.; Kleijn, H.; Westmijze, H.; Meijer, J.; Vermeer, P. Recl. Trav. Chim. Pays-Bas 1982, 101, 405-409. doi:10.1002/recl.19821011107

28. Ruitenberg, K.; Kleijn, H.; Elsevier, C. J.; Meijer, J.; Vermer, P. Tetrahedron Lett. 1981, 22, 1451-1452. doi:10.1016/S0040-4039(01)90347-8

29. Pasto, D. J.; Yank, S. H. J. Org. Chem. 1989, 54, 3544-3549. doi:10.1021/jo00276a010

30. Elsevier, C. J.; Stehouver, P. M.; Westmijze, H.; Vermeer, P. J. Org. Chem. 1983, 48, 1103-1105. doi:10.1021/jo00155a036

31. Ruitenberg, K.; Westmijze, H.; Kleijn, H.; Vermeer, P. J. Organomet. Chem. 1984, 277, 227-234. doi:10.1016/0022-328X(84)80703-2

32. Mkryan, G. M.; Mkrtchyan, A. M.; Gasparyan, S. M.; Voskanyan, E. S.; Nazaryan, A. A. Zh. Org. Khim. 1974, 10, 1878-1881. J. Org. Chem. USSR, 1974, 10, 1889-1892.

33. Mkryan, G. M.; Gasparyan, S. M.; Papazyan, N. A.; Voskanyan, E. S. Zh. Org. Khim. 1972, 8, 1397-1400. J. Org. Chem. USSR, 1972, 8, 1418-1420.

34. Polizzi, C.; Consoloni, C.; Lardicci, L.; Caporusso, A. M. J. Organomet. Chem. 1991, 417, 289-304. doi:10.1016/0022-328X(91)80179-N

35. Caporusso, A. M.; Aronica, L. A.; Geri, R.; Gori, M. J. Organomet. Chem. 2002, 648, 109-118. doi:10.1016/S0022-328X(01)01438-3

36. Barlet, R. Bull. Soc. Chim. Fr. 1979, 132-139.

37. Hara, S.; Sato, Y.; Suzuki, A. Chem. Lett. 1982, 11, 1289-1290. doi:10.1246/cl.1982.1289

38. Khrimyan, A. P.; Badanyan, S. O. Arm. Khim. Zh. 1978, 31, 682-686.

39. Hopf, H. Chem. Ber. 1971, 104, 3087-3095. doi:10.1002/cber.19711041013

40. Tolstikov, G. A.; Romanova, T. Yu.; Kuchin, A. V. J. Organomet. Chem. 1985, 285, 71-82. doi:10.1016/0022-328X(85)87358-7
41. Tolstikov, G. A.; Romanova, T. Yu.; Muslukhov, R. R.; Kuchin, A. V. Izv. Akad. Nauk SSSR, Ser. Khim. 1987, 1331-1337. Bull. Acad. Science USSR, 1987, 36, 1227-1233. doi:10.1007/BF00956668

42. Toda, F.; Tanaka, K.; Nawata, H. J. Chem. Soc., Perkin Trans. 1 1994, 2043-2044. doi:10.1039/p19940002043

43. Coulomb-Delbecq, F.; Goré, J. Bull. Soc. Chim. Fr. 1976, 541-549.

44. Toda, F.; Takehira, Y. J. Chem. Soc., Chem. Commun. 1975, 174. doi:10.1039/c39750000174

45. Petasis, N. A.; Hu, Y.-H. J. Org. Chem. 1997, 62, 782-783. doi:10.1021/jo9620876

46. Coffman, D. D.; Carothers, W. H. J. Am. Chem. Soc. 1933, 55, 2040-2047. doi:10.1021/ja01332a041

47. Patel, A. N. J. Org. Chem. USSR 1977, 13, 1902-1904.

48. Hopf, H.; Sherburn, M. S. Angew. Chem., Int. Ed. 2012, 51, 2298-2338. doi:10.1002/anie.201102987

49. Toda, F.; Toshikaru, H. Patent Abstr. Jap. 07-24762, Sept 26, 1995.

50. Toda, F.; Motomura, H.; Oshima, H. Bull. Chem. Soc. Jpn. 1974, 47, 467-470. doi:10.1246/bcsj.47.467

51. Saalfrank, R. W.; Welch, A.; Haubner, M.; Bauer, U. Liebigs Ann. 1996, 171-181. doi:10.1002/jlac.199619960205

52. Cai, B.; Blackburn, G. M. Synth. Commun. 1997, 27, 3943-3949.

53. Milsosevic, S.; Banide, E. V.; Müller-Bunz, H.; Gilheany, D. G.; McGlinchey, M. J. Organometallics 2011, 30, 3804-3817. doi:10.1021/om200357v

54. Banert, K.; Köhler, F.; Melzer, A.; Scharf, I.; Rheinwald, G.; Rüffer, T.; Lang, H. Synthesis 2011, 1561-1568. doi:10.1055/s-0030-1260000

55. Raj, C. P.; Braverman, S. Synth. Commun. 1999, 29, 2629-2637. doi:10.1080/00397919908086424

56. Braverman, S.; Kumar, E. V. K. S.; Cherkinsky, M.; Sprecher, M.; Goldberg, I. Tetrahedron Lett. 2000, 41, 6923-6927. doi:10.1016/S0040-4039(00)01148-5

57. Braverman, S.; Kumar, E. V. K. S.; Cherkinsky, M.; Sprecher, M.; Goldberg, I. Tetrahedron 2005, 61, 3547-3557. doi:10.1016/j.tet.2005.01.114

58. Bohlmann, F.; Kieslich, K. Chem. Ber. 1954, 87, 1363-1372. doi:10.1002/cber.19540870926

59. Bohlmann, F.; Kieslich, K. Chem. Ber. 1955, 88, 1211-1218. doi:10.1002/cber.19550880811

60. Negi, T.; Kaneda, T.; Mizuno, H.; Toyoda, T.; Sakata, Y.; Misumi, S. Bull. Chem. Soc. Jpn. 1974, 47, 2398-2405. doi:10.1246/bcsj.47.2398

61. Kuwatani, Y.; Yamamoto, G.; Oda, M.; lyoda, M. Bull. Chem. Soc. Jpn. 2005, 78, 2188-2208. doi:10.1246/bcsj.78.2188

62. Iyoda, M.; Kuwatani, Y.; Oda, M.; Kai, Y.; Kanehisa, N.; Kasai, N. Angew. Chem., Int. Ed. Engl. 1990, 29, 1062-1064. doi:10.1002/anie.199010621

63. Iyoda, M.; Oda, M.; Kai, Y.; Kanehisa, N.; Kasai, N. Chem. Lett. 1990, 19, 2149-2152. doi:10.1246/cl.1990.2149

64. Parkhurst, R. R.; Swager, T. M. Synlett 2011, 1519-1522. doi:10.1055/s-0030-1260777

65. Pal, R.; Clark, R. J.; Manoharan, M.; Alabugin, I. V. J. Org. Chem. 2010, 75, 8689-8692. doi:10.1021/jo101838a

66. Yan, X.; Lai, C.; Xi, C. Chem. Commun. 2009, 6026-6028. doi:10.1039/B912175G

67. Maraval, V.; Duhayon, C.; Coppel, Y.; Chauvin, R. Eur. J. Org. Chem. 2008, 5144-5156. doi:10.1002/ejoc.200800711

68. Banide, E. V.; Oulié, P.; Müller-Bunz, H.; McGlinchey, M. J. Organometallics 2008, 27, 5657-5664. doi:10.1021/om8005855 
69. Lorbach, A.; Reus, C.; Bolte, M.; Lerner, H.-W.; Wagner, M. Adv. Synth. Catal. 2010, 352, 3443-3449. doi:10.1002/adsc. 201000560

70. Ballard, D. H.; Brennan, T.; Gilman, H. J. Organomet. Chem. 1970, 22, 583-587. doi:10.1016/S0022-328X(00)87712-8

71. Sekiguchi, A.; Ebata, K.; Terui, Y.; Sakurai, H. Chem. Lett. 1991, 20, 1417-1420. doi:10.1246/cl.1991.1417

72. Sakurai, H.; Ebata, K.; Kabuto, C.; Sekiguchi, A. J. Am. Chem. Soc. 1990, 112, 1799-1803. doi:10.1021/ja00161a023

73. Sakamoto, K.; Saeki, T.; Sakurai, H. Chem. Lett. 1993, 22, 1675-1678. doi:10.1246/cl.1993.1675

74. Priester, W.; West, R. J. Am. Chem. Soc. 1976, 98, 8426-8432. doi:10.1021/ja00442a019

75. Klein, J.; Becker, J. Y. J. Chem. Soc., Perkin Trans. 2 1973, 599-603. doi:10.1039/p29730000599

76. Fritz, G.; Beetz, A. G.; Matern, E.; Peters, K.; Peters, E.-M.; von Schnering, H. G. Z. Anorg. Allg. Chem. 1994, 620, 136-144. doi:10.1002/zaac.19946200122

77. Weissensteiner, W.; Schuster, I. I.; Blount, J. F.; Mislow, K. J. Am. Chem. Soc. 1986, 108, 6664-6668. doi:10.1021/ja00281a036

78. Baudouy, R.; Goré, J. Tetrahedron Lett. 1974, 15, 1593-1596. doi:10.1016/S0040-4039(01)82528-4

79. Baudouy, R.; Goré, J. Bull. Soc. Chim. Fr. 1975, 2166-2170.

80. Poonoth, M.; Krause, N. Adv. Synth. Catal. 2009, 351, 117-122. doi:10.1002/adsc.200800469

81. Aurrecoechea, J. M.; Alonso, E.; Solay, M. Tetrahedron 1998, 54, 3833-3850. doi:10.1016/S0040-4020(98)00110-0

82. Marshall, J. A.; Adams, N. D. J. Org. Chem. 1999, 64, 5201-5204. doi:10.1021/jo9823083

83. Ghera, E.; Shoua, S. Tetrahedron Lett. 1974, 15, 3843-3846. doi:10.1016/S0040-4039(01)92024-6

84. Braverman, S.; Zafrani, Y.; Rahimipour, S. Tetrahedron Lett. 2001, 42, 2911-2914. doi:10.1016/S0040-4039(01)00322-7

85. Yu, H.; Lee, P. H. J. Org. Chem. 2008, 73, 5183-5186. doi:10.1021/jo800594y

86. Bildstein, B.; Kopacka, H.; Schweiger, M.; Ellmerer-Müller, E.; Ongania, K.-H.; Wurst, K. Organometallics 1996, 15, 4398-4406. doi:10.1021/om960170d

87. Sparks, W. J.; Peppel, W. J.; Marvel, C. S. J. Am. Chem. Soc. 1937, 59, 1351-1352. doi:10.1021/ja01286a053

88. Toda, F.; Takahara, Y. Bull. Chem. Soc. Jpn. 1976, 49, 2515-2517. doi:10.1246/bcsj.49.2515

89. Toda, F.; Tanaka, K.; Tsukada, H.; Shimanouchi, H.; Sasada, Y. Chem. Lett. 1979, 8, 1381-1382. doi:10.1246/cl.1979.1381

90. Hauptmann, H. Tetrahedron Lett. 1975, 16, 1931-1934. doi:10.1016/S0040-4039(00)72325-2

91. Bildstein, B.; Schweiger, M.; Kopacka, H.; Ongania, K.-H.; Wurst, K. Organometallics 1998, 17, 2414-2424. doi:10.1021/om980023a

92. Powell, D. L.; Klaeboe, P.; Christensen, D. H.; Hopf, H. Spectrochim. Acta, Part A 1973, 29, 7-16. doi:10.1016/0584-8539(73)80003-0

93. Eriksson, A.; Brunvoll, J.; Hagen, G.; Cyvin, S. J.; Bjøseth, A.; Powell, D. L. Acta Chem. Scand., Ser. A 1974, A28, 439-441. doi:10.3891/acta.chem.scand.28a-0439

94. Trætteberg, M.; Paulen, G.; Hopf, H. Acta Chem. Scand. 1973, 27, 2227-2229. doi:10.3891/acta.chem.scand.27-2227

95. Pedersen, B.; Schaug, J.; Hopf, H. Acta Chem. Scand., Ser. A 1974, A28, 846-850. doi:10.3891/acta.chem.scand.28a-0846

96. Bieri, G.; Burger, F.; Heilbronner, E.; Maier, J. P. Helv. Chim. Acta 1977, 60, 2213-2233. doi:10.1002/hlca.19770600714
97. Bischof, P.; Gleiter, R.; Hopf, H.; Lenich, F. T. J. Am. Chem. Soc. 1975, 97, 5467-5472. doi:10.1021/ja00852a025

98. Brogli, F.; Crandall, J. K.; Heilbronner, E.; Kloster-Jensen, E.; Sojka, S. A. J. Electron Spectrosc. Relat. Phenom. 1973, 2, 455-456. doi:10.1016/0368-2048(73)80036-2

99. Delwiche, J.; Praet, M.-T.; Caprace, C.; Franskin-Hubin, M.-J.; Natalis, P.; Collin, J. E.; Hopf, H.

J. Electron Spectrosc. Relat. Phenom. 1979, 16, 35-43. doi:10.1016/0368-2048(79)85003-3

100. We thank Professor P. G. Jones (University of Braunschweig) for carrying out this search.

101.Jones, P. G.; Bubenitschek, P.; Hopf, H.; Stamm, R. Acta Crystallogr., Sect. E 2002, 58, o277-0278. doi:10.1107/S1600536802002696

102. Eaton, B. E.; Rollman, B.; Kaduk, J. A. J. Am. Chem. Soc. 1992, 114, 6245-6246. doi:10.1021/ja00041a052

103.Jones, P. G.; Bubenitschek, P.; Hopf, H.; Stamm, R. Acta Crystallogr., Sect. E 2003, 59, o119-0120. doi:10.1107/S1600536802022870

104. Groth, P. Acta Chem. Scand. 1973, 27, 3302-3306. doi:10.3891/acta.chem.scand.27-3302

105.Kaupp, G.; Schmeyers, J.; Kato, M.; Tanaka, K.; Harada, N.; Toda, F. J. Phys. Org. Chem. 2001, 14, 444-452. doi:10.1002/poc.388

106. Toda, F.; Tanaka, K.; Tamashima, T.; Kato, M. Angew. Chem., Int. Ed. 1998, 37, 2724-2727. doi:10.1002/(SICI)1521-3773(19981016)37:19<2724::AID-ANIE2724> 3.3.CO;2-P

107.Poonoth, M.; Schürmann, M.; Preut, H.; Krause, N. Acta Crystallogr., Sect. E 2007, 63, 04402. doi:10.1107/S1600536807051835

108. Huntsman, W. D.; Wristers, H. J. J. Am. Chem. Soc. 1963, 85, 3308-3309. doi:10.1021/ja00903a065

109. Huntsman, W. D.; Wristers, H. J. J. Am. Chem. Soc. 1967, 89, 342-347. doi:10.1021/ja00978a030

110.Viola, A.; Collins, J. J.; Filipp, N. Tetrahedron 1981, 37, 3765-3811. doi:10.1016/S0040-4020(01)98877-5

111.Sakai, S. J. Phys. Chem. A 2006, 110, 9443-9450. doi:10.1021/jp061828w

112. Miller, C. H.; Tang, W.; Tranter, R. S.; Brezinsky, K. J. Phys. Chem. A 2006, 110, 3605-3613. doi:10.1021/jp055990v

113.Dai, S.; Pappas, R. S.; Chen, G. F.; Guo, Q. X.; Wang, J. T.; Williams, F. J. Am. Chem. Soc. 1989, 111, 8759-8761. doi:10.1021/ja00205a050

114.Schneider, R. Ph.D. Thesis, Karlsruhe, Germany, 1977. Unpublished results.

115.Pasto, D. J.; Kong, W. J. Org. Chem. 1989, 54, 4028-4033. doi:10.1021/jo00278a010

116.Pasto, D. J.; Yang, S. H. J. Org. Chem. 1989, 54, 3978-3981. doi:10.1021/jo00277a042

117. Kleveland, K.; Skattebøl, L. J. Chem. Soc., Chem. Commun. 1973, 432-433. doi:10.1039/C39730000432

118. Hopf, H.; Trabert, L. Liebigs Ann. Chem. 1980, 1786-1800. doi:10.1002/jlac.198019801118

119. Toda, F. Eur. J. Org. Chem. 2000, 1377-1386. doi:10.1002/(SICI)1099-0690(200004)2000:8<1377::AID-EJOC1377> 3.0.CO;2-I

120.Tanaka, K.; Takamoto, N.; Tezuka, Y.; Kato, M.; Toda, F. Tetrahedron 2001, 57, 3761-3767. doi:10.1016/S0040-4020(01)00249-6 
121.Oulié, P.; Altes, L.; Milosevic, S.; Bouteille, R.; Müller-Bunz, H.; McGlinchey, M. J. Organometallics 2010, 29, 676-686. doi:10.1021/om9009872

122.Schön, G.; Hopf, H. Liebigs Ann. Chem. 1981, 165-180. doi:10.1002/jlac.198119810123

123.Boan, C.; Skattebøl, L. J. Chem. Soc., Perkin Trans. 11978 , 1568-1572. doi:10.1039/p19780001568

124.Hopf, H. Angew. Chem., Int. Ed. Engl. 1972, 11, 419-420. doi:10.1002/anie.197204191

125. Hopf, H.; Lenich, F. T. Chem. Ber. 1974, 107, 1891-1902. doi:10.1002/cber.19741070613

126.Böhm, I.; Herrmann, H.; Menke, K.; Hopf, H. Chem. Ber. 1978, 111, 523-527. doi:10.1002/cber.19781110211

127.Dix, I.; Hopf, H.; Satyanarayana, T. B. N.; Ernst, L. Beilstein J. Org. Chem. 2010, 6, 932-937. doi:10.3762/bjoc.6.104

128. Kleveland, K.; Skattebøl, L. Acta Chem. Scand., Ser. B 1975, B29, 827-830. doi:10.3891/acta.chem.scand.29b-0827

129.Billeb, G.; Bootz, K.; Neumann, W. P.; Steinhoff, G. J. Organomet. Chem. 1991, 406, 303-308. doi:10.1016/0022-328X(91)83118-N

130.Marx, R.; Neumann, W. P.; Hillner, K. Tetrahedron Lett. 1984, 25 , 625-628. doi:10.1016/S0040-4039(00)99955-6

131.Schriewer, M.; Neumann, W. P. J. Am. Chem. Soc. 1983, 105 , 897-901. doi:10.1021/ja00342a043

132.Schriewer, M.; Neumann, W. P. Angew. Chem., Int. Ed. Engl. 1981, 20, 1019-1021. doi:10.1002/anie.198110191

133.Bertini, F.; Wit, J. B. M.; Ünal, M.; de Kanter, F. J. J.; Schakel, M.; Slootweg, J. C.; Ehlers, A. W.; Nijbacker, T.; Komen, C. M. D.; Lutz, M.; Spek, A. L.; Lammertsma, K. Adv. Synth. Catal. 2009, 351, 1132-1138. doi:10.1002/adsc.200900005

134.Wit, J. B. M.; van Eijkel, G. T.; de Kanter, F. J. J.; Schakel, M.; Ehlers, A. W.; Lutz, M.; Spek, A. L.; Lammertsma, K. Angew. Chem., Int. Ed. 1999, 38, 2596-2599. doi:10.1002/(SICI)1521-3773(19990903)38:17<2596::AID-ANIE2596> 3.0.CO;2-I

135. Heinrich, F.; Lüttke, W. Angew. Chem., Int. Ed. Engl. 1972, 11, 234-236. doi:10.1002/anie.197202341

136. Eichenberger, H.; Wolf, H. R.; Jeger, O. Helv. Chim. Acta 1977, 60, 743-767. doi:10.1002/hlca.19770600308

137.Siegel, H.; Eisenhuth, L.; Hopf, H. Chem. Ber. 1985, 118, 597-612. doi:10.1002/cber.19851180219

138. Hopf, H.; Stamm, R., in press.

139.Pasto, D. J.; Yang, S. H.; Muellerleile, J. A. J. Org. Chem. 1992, 57, 2976-2978. doi:10.1021/jo00036a047

140.Santelli-Rouvier, C.; Santelli, M. Synthesis 1983, 429-442. doi:10.1055/s-1983-30367

141. Roumestant, M. L.; Malacria, M.; Goré, J.; Grimaldi, J.; Bertrand, M. Synthesis 1976, 755-757. doi:10.1055/s-1976-24192 And references cited therein.

142. Hopf, H.; Lenich, F. T. Chem. Ber. 1973, 106, 3461-3462. doi:10.1002/cber.19731061031

143.Pasto, D. J.; Huang, N.-Z. J. Org. Chem. 1985, 50, 4465-4467. doi:10.1021/jo00223a010

144.Chen, B.; Lu, Z.; Chai, G.; Fu, C.; Ma, S. J. Org. Chem. 2008, 73, 9486-9489. doi:10.1021/jo801809j

145. Hopf, H. Tetrahedron Lett. 1970, 11, 1107-1109. doi:10.1016/S0040-4039(01)97920-1

146. Roth, W. R.; Kowalczik, U.; Maier, G.; Reisenauer, H. P.; Sustmann, R.; Müller, W. Angew. Chem., Int. Ed. Engl. 1987, 26, 1285-1287. doi:10.1002/anie.198712851
147.Roth, W. R.; Adamczak, O.; Breuckmann, R.; Lennartz, H.-W.; Boese, R. Chem. Ber. 1991, 124, 2499-2521. doi:10.1002/cber.19911241121

148. Ried, W.; Marhold, A. Chem. Ber. 1973, 106, 2455-2459. doi:10.1002/cber.19731060805

149. Skattebøl, L. J. Org. Chem. 1966, 31, 2789-2794. doi:10.1021/jo01347a014

150.Becher, G.; Skattebøl, L. Tetrahedron Lett. 1979, 20, 1261-1264. doi:10.1016/S0040-4039(01)86119-0

151. Lahrech, M.; Hacini, S.; Parrain, J.-L.; Santelli, M. Tetrahedron Lett. 1997, 38, 3395-3398. doi:10.1016/S0040-4039(97)00628-X

152.Emptoz, G.; Vo-Quang, L.; Vo-Quang, Y. Bull. Soc. Chim. Fr. 1965, 2653-2655.

153. Romanov, O. E.; Zubritskii, L. M.; Bal'yan, K. V. Zh. Org. Khim. 1977, 13, 468-469.

J. Org. Chem. USSR, 1977, 13, 428-429 and earlier publications in the series.

154.Pornet, J.; Randrianoelina, B.; Miginac, L. J. Organomet. Chem. 1979, 174, 1-13. doi:10.1016/S0022-328X(00)96157-6

155.Boss, C.; Keese, R. Helv. Chim. Acta 1997, 80, 414-420. doi:10.1002/hlca.19970800207

156. Aubert, P.; Princet, B.; Pornet, J. Synth. Commun. 1997, 27, 2615-2625. doi:10.1080/00397919708004131

157.Maercker, A.; Wunderlich, H.; Girreser, U. Tetrahedron 1996, 52, 6149-6172. doi:10.1016/0040-4020(96)00235-9

158. Cao, H.; Flippen-Anderson, J.; Cook, J. M. J. Am. Chem. Soc. 2003, 125, 3230-3231. doi:10.1021/ja021159+

159. Miebach, T.; Wuster, H.; Brinker, U. H. J. Org. Chem. 1993, 58, 6520-6523. doi:10.1021/jo00075a067

160. Richey, H. G., Jr.; Kossa, W. C., Jr. Tetrahedron Lett. 1969, 10, 2313-2314. doi:10.1016/S0040-4039(01)88150-8

161.Prasad, A. S. B.; Eick, H.; Knochel, P. J. Organomet. Chem. 1998, 562, 133-139. doi:10.1016/S0022-328X(97)00746-8

162.Tseng, H.-R.; Luh, T.-Y. J. Org. Chem. 1996, 61, 8685-8686. doi:10.1021/j0961514c

163. Barbot, F.; Miginiac, P. J. Organomet. Chem. 1981, 222, 1-15. doi:10.1016/S0022-328X(00)89012-9

164.Gevorgyan, V.; Kadowaki, C.; Salter, M. M.; Kodota, I.; Saito, S.; Yamamoto, Y. Tetrahedron 1997, 53, 9097-9106. doi:10.1016/S0040-4020(97)00602-9

165.Djahanbini, D.; Cazes, B.; Goré, J. Tetrahedron 1987, 43, 3441-3452. doi:10.1016/S0040-4020(01)81635-5

166. Djahanbini, D.; Cazes, B.; Goré, J. Tetrahedron Lett. 1984, 25 , 203-206. doi:10.1016/S0040-4039(00)99840-X

167. Ogasawara, M.; Ikeda, H.; Nagano, T.; Hayashi, T. Org. Lett. 2001, 3, 2615-2617. doi:10.1021/ol016320k

168. Lee, K.; Seomoon, D.; Lee, P. H. Angew. Chem., Int. Ed. 2002, 41, 3901-3903. doi:10.1002/1521-3773(20021018)41:20<3901::AID-ANIE3901>3.0.C O;2-S

169.Searles, S.; Li, Y.; Nassim, B.; Lopez, M.-T. R.; Tran, P. T.; Crabbé, P. J. Chem. Soc., Perkin Trans. 1 1984, 747-751. doi:10.1039/p19840000747

170.Skattebøl, L.; Solomon, S. J. Am. Chem. Soc. 1965, 87, 4506-4513. doi:10.1021/ja00948a019

171.Roth, W. R.; Scholz, B. P.; Breuckmann, R.; Jelich, K.; Lennartz, H.-W. Chem. Ber. 1982, 115, 1934-1946. doi:10.1002/cber.19821150523

172. Roth, W. R.; Erker, G. Angew. Chem., Int. Ed. Engl. 1973, 12, 503-504. doi:10.1002/anie.197305031 
173.Roth, W. R.; Heiber, M.; Erker, G. Angew. Chem., Int. Ed. Engl. 1973, 12, 504-505. doi:10.1002/anie.197305041

174.Grimme, W.; Rother, H.-J. Angew. Chem., Int. Ed. Engl. 1973, 12, 505-506. doi:10.1002/anie.197305052

175.Jug, K.; Iffert, R. Theor. Chim. Acta 1982, 62, 1128-1138.

176.Kaufmann, D.; de Meijere, A. Chem. Ber. 1984, 117, 1128-1138. doi:10.1002/cber.19841170327

177.Kawamura, T.; Inagaki, F.; Narita, S.; Takahashi, Y.; Hirata, S.; Kitagaki, S.; Mukai, C. Chem.-Eur. J. 2010, 16, 5173-5183. doi:10.1002/chem.200903568

178.Lian, X.; Ma, S. Chem.-Eur. J. 2010, 16, 7960-7964. doi:10.1002/chem.201000992

179.Lu, P.; Ma, S. Chin. J. Chem. 2010, 28, 1600-1606. doi:10.1002/cjoc.201090271

180.Shu, W.; Jia, G.; Ma, S. Angew. Chem., Int. Ed. 2009, 48, 2788-2791. doi:10.1002/anie.200805422

181.Deng, Y.; Shi, Y.; Ma, S. Org. Lett. 2009, 11, 1205-1209. doi:10.1021/ol802794t

182.Lu, P.; Ma, S. Org. Lett. 2007, 9, 2095-2097. doi:10.1021/ol070632r

183.Jiang, X.; Cheng, X.; Ma, S. Angew. Chem., Int. Ed. 2006, 45 , 8009-8013. doi:10.1002/anie.200602847

184.Cheng, J.; Jiang, X.; Ma, S. Org. Lett. 2011, 13, 5200-5203. doi:10.1021/ol202074e

185.Hong, Y.-T.; Yoon, S.-K.; Kang, S.-K.; Yu, C.-M. Eur. J. Org. Chem. 2004, 4628-4635. doi:10.1002/ejoc.200400361

186.Kang, S.-K.; Baik, T.-G.; Kulak, A. N.; Ha, Y.-H.; Lim, Y.; Park, J. J. Am. Chem. Soc. 2000, 122, 11529-11530. doi:10.1021/ja001597।

187.Kang, S.-K.; Ha, Y.-H.; Kim, D.-H.; Lim, Y.; Jung, J. Chem. Commun. 2001, 1306-1307. doi:10.1039/b101870l

188.Ma, S.; Lu, P.; Lu, L.; Hou, H.; Wei, J.; He, Q.; Gu, Z.; Jiang, X.; Jin, X. Angew. Chem., Int. Ed. 2005, 44, 5275-5278. doi:10.1002/anie.200501350

189. Matsumi, N.; Chujo, Y. Polym. Bull. 1999, 43, 117-120. doi:10.1007/s002890050541

190.Liu, K.; Kim, H.; Ghosh, P.; Akhmedov, N. G.; Williams, L. J. J. Am. Chem. Soc. 2011, 133, 14968-14971. doi:10.1021/ja207496p

191. Hashmi, A. S. K.; Schwarz, L.; Bolte, M. Eur. J. Org. Chem. 2004, 1923-1935. doi:10.1002/ejoc.200300792

192. Mochizuki, K.; Tomita, I. Polym. Bull. 2006, 56, 331-338. doi:10.1007/s00289-006-0503-9

193. Matsumi, N.; Chujo, Y. Polym. Bull. 1997, 39, 295-302. doi:10.1007/s002890050151 And references cited therein.

194. Brinker, U. H.; Fleischhauer, I. Tetrahedron 1981, 37, 4495-4502. doi:10.1016/0040-4020(81)80016-6

And references cited therein.

195.Ben-Efraim, D. A.; Sondheimer, F. Tetrahedron Lett. 1963, 4, 313-315. doi:10.1016/S0040-4039(01)90627-6

196. Lehrich, F.; Hopf, H. Tetrahedron Lett. 1987, 28, 2697-2700. doi:10.1016/S0040-4039(00)96184-7

197.Lehrich, F.; Hopf, H.; Grunenberg, J. Eur. J. Org. Chem. 2011, 2705-2718. doi:10.1002/ejoc.201001508

198. Märkl, G.; Attenberger, P.; Kellner, J. Tetrahedron Lett. 1988, 29 , 3651-3654. doi:10.1016/S0040-4039(00)82145-0

199.Saalfrank, R. W.; Haubner, M.; Deutscher, C.; Bauer, W. Eur. J. Org. Chem. 1999, 2367-2372. doi:10.1002/(SICI)1099-0690(199909)1999:9<2367::AID-EJOC2367> 3.3.CO;2-0
200. Odermatt, S.; Alonso-Gómez, J. L.; Seiler, P.; Cid, M. M.; Diederich, F. Angew. Chem., Int. Ed. 2005, 44, 5074-5078. doi:10.1002/anie.200501621

201.Bowes, C. M.; Montecalvo, D. F.; Sondheimer, F. Tetrahedron Lett. 1973, 14, 3181-3184. doi:10.1016/S0040-4039(00)79805-4

202. Inanaga, J.; Sujimoto, Y.; Hanamoto, T. Tetrahedron Lett. 1992, 33, 7035-7038. doi:10.1016/S0040-4039(00)60926-7

203.Sato, E.; Yokozawa, T.; Endo, T. Macromol. Rapid Commun. 1994, 15, 607-611. doi:10.1002/marc.1994.030150706

204. Reynolds, K. A.; Dopico, P. G.; Brady, M. S.; Finn, M. G. J. Org. Chem. 1997, 62, 2564-2573. doi:10.1021/jo961000d

205. Rauss-Godineau, J.; Chodkiewicz, W.; Cadiot, P. Bull. Soc. Chim. Fr. 1966, 2877-2884.

206. Su, K.-J.; Mieusset, J.-L.; Arion, V. B.; Knoll, W.; Brecker, L.; Brinker, U. H. J. Org. Chem. 2010, 75, 7494-7497. doi:10.1021/j01017298

207.Lee, P. H.; Mo, J.; Kang, D.; Eom, D.; Park, C.; Lee, C.-H.; Jung, Y. M.; Hwang, H. J. Org. Chem. 2011, 76, 312-315. doi:10.1021/j01020085

208.Ji, K.-G.; Shu, X.-Z.; Chen, J.; Zhao, S.-C.; Zheng, Z.-J.; Lu, L.; Liu, X.-Y.; Liang, Y.-M. Org. Lett. 2008, 10, 3919-3922. doi:10.1021/ol8015463

209.Bi, H.-P.; Liu, X.-Y.; Gou, F.-R.; Guo, L.-N.; Duan, X.-H.; Liang, Y.-M. Org. Lett. 2007, 9, 3527-3529. doi:10.1021/ol071385u

210.Amombo, M. O.; Hausherr, A.; Reissig, H.-U. Synlett 1999, 1871-1874. doi:10.1055/s-1999-2966

211.Janßen, C. E.; Krause, N. Eur. J. Org. Chem. 2005, 2322-2329. doi:10.1002/ejoc.200500096

212. Thorand, S.; Vögtle, F.; Krause, N. Angew. Chem., Int. Ed. 1999, 38, 3721-3723. doi:10.1002/(SICI)1521-3773(19991216)38:24<3721::AID-ANIE3721> 3.0.CO;2-9

213.Takahashi, Y.; Tomura, M.; Yoshida, K.; Murata, S.; Tomioka, H. Angew. Chem., Int. Ed. 2000, 39, 3478-3480. doi:10.1002/1521-3773(20001002)39:19<3478::AID-ANIE3478>3.0.C O;2-L

214.Miyaki, N.; Tomita, I.; Kido, J.; Endo, T. Macromolecules 1997, 30, 4504-4506. doi:10.1021/ma9702410

215.Dulcère, J. P.; Agati, V.; Faure, R. J. Chem. Soc., Chem. Commun. 1993, 270-271. doi:10.1039/C39930000270

216. Birsa, M. L.; Jones, P. G.; Braverman, S.; Hopf, H. Synlett 2005, 640-642. doi:10.1055/s-2005-863715

217. Birsa, M. L.; Hopf, H. Phosphorus, Sulfur Silicon Relat. Elem. 2005, 180, 1453-1454. doi:10.1080/10426500590913041

218. Hartzler, H. D. J. Am. Chem. Soc. 1971, 93, 4527-4531. doi:10.1021/ja00747a031

219. Hartzler, H. D. J. Am. Chem. Soc. 1966, 88, 3155-3156. doi:10.1021/ja00965a062

220. Kaftory, M.; Agmon, I.; Ladika, M.; Stang, P. J. J. Am. Chem. Soc. 1987, 109, 782-787. doi:10.1021/ja00237a024

221.Stang, P. J.; Learned, A. E. J. Chem. Soc., Chem. Commun. 1988, 301-302. doi:10.1039/c39880000301

222.Learned, A. E.; Arif, A. M.; Stang, P. J. J. Org. Chem. 1988, 53, 3122-3123. doi:10.1021/jo00248a043

223. Iyoda, M.; Kuwatani, Y.; Oda, M. J. Am. Chem. Soc. 1989, 111, 3761-3762. doi:10.1021/ja00192a051

224. Hopf, H.; Maas, G. Angew. Chem., Int. Ed. Engl. 1992, 31, 931-954. doi:10.1002/anie.199209313 
225. Hopf, H.; Maas, G. Chapter 21. Synthesis and Transformation of Radialenes. In The chemistry of dienes and polyenes, Vol. I; Rappoport, Z., Ed.; The Chemistry of Functional Groups; Wiley-Interscience: Chichester, 1997; pp 927-977. doi:10.1002/0470857218.ch21

226. Berkovich-Yellin, Z.; Lahav, M.; Leiserowitz, L. J. Am. Chem. Soc. 1974, 96, 918-920. doi:10.1021/ja00810a048

227.Buddrus, J.; Bauer, H.; Herzog, H. Chem. Ber. 1988, 121, 295-297. doi:10.1002/cber.19881210215

228. Blickle, P.; Hopf, H. Tetrahedron Lett. 1978, 19, 449-452. doi:10.1016/S0040-4039(01)91451-0

229.Delas, C.; Urabe, H.; Sato, F. Tetrahedron Lett. 2001, 42, 4147-4150. doi:10.1016/S0040-4039(01)00666-9

230.Hopf, H.; Gottschild, D.; Lenk, W. Isr. J. Chem. 1985, 26, 79-87.

231.Hara, R.; Ura, Y.; Huo, S.; Kasei, K.; Suzuki, N.; Takahashi, T. Inorg. Chim. Acta 2000, 300-302, 741-748.

doi:10.1016/S0020-1693(99)00601-5 See for another approach to prepare $\mathbf{4 3 0}$.

232. Mebane, R. C.; Schuster, G. B. J. Org. Chem. 1983, 48, 810-812. doi:10.1021/jo00154a011

233. Heldeweg, R. F.; Hogeveen, H. J. Org. Chem. 1978, 43, 1916-1920. doi:10.1021/jo00404a016

234. Hashmi, A. S. K.; Szeimies, G. Chem. Ber. 1994, 127, 1075-1089. doi:10.1002/cber.19941270617

235. Tobe, Y.; Fujii, T.; Matsumoto, H.; Tsumuraya, K.; Noguchi, D.; Nakagawa, N.; Sonoda, M.; Naemura, K.; Achiba, Y.; Wakabayashi, T. J. Am. Chem. Soc. 2000, 122, 1762-1775. doi:10.1021/ja993314z

236.Skattebøl, L. Tetrahedron Lett. 1961, 2, 167-172. doi:10.1016/S0040-4039(01)84059-4

237. Harris, J. F., Jr. Tetrahedron Lett. 1965, 6, 1359-1362. doi:10.1016/S0040-4039(00)90070-4

238. Dehmlow, E. V.; Stiehm, T. Tetrahedron Lett. 1990, 31, 1841-1844. doi:10.1016/S0040-4039(00)98800-2

239. Dehmlow, E. V.; Ezimora, G. C. Tetrahedron Lett. 1970, 11, 4047-4050. doi:10.1016/S0040-4039(01)98661-7

240. Irngartinger, H.; Jäger, H.-U. Tetrahedron Lett. 1976, 17, 3595-3596. doi:10.1016/S0040-4039(00)92968-X

241.Anet, F. A. L.; Yavari, I. J. Am. Chem. Soc. 1977, 99, 7640-7649. doi:10.1021/ja00465a036

242. Charrier, C.; Dorman, D. E.; Roberts, J. D. J. Org. Chem. 1973, 38, 2644-2650. doi:10.1021/jo00955a015

243.Baird, M. S.; Reese, C. B. Tetrahedron 1976, 32, 2153-2156. doi:10.1016/0040-4020(76)85127-7

244.Roth, W. R.; Schaffers, T.; Heiber, M. Chem. Ber. 1992, 125, 739-749. doi:10.1002/cber.19921250330

245.Shimizu, T.; Kamigata, N.; Ikuta, S. J. Mol. Struct.: THEOCHEM 1996, 369, 127-135. doi:10.1016/S0166-1280(96)04643-X See for the confomational analysis of various cyclic bisallenes by theoretical calculations.

246. Yavari, I.; Baharfar, R.; Nori-Shargh, D. J. Mol. Struct.: THEOCHEM 1997, 393, 167-170. doi:10.1016/S0166-1280(96)04848-8

247. Yavari, I.; Baharfar, R.; Nori-Shargh, D.; Shaabani, A. J. Chem. Res., Synop. 1997, 162-163. doi:10.1039/a608314e

248.Böhm, M. C.; Gleiter, R. Chem. Ber. 1978, 111, 3516-3518. doi:10.1002/cber.19781111028

249.Becker, J. Y. Tetrahedron Lett. 1976, 17, 2159-2162. doi:10.1016/S0040-4039(00)93146-0
250.Baker, R.; Garratt, P. J.; Nicolaou, K. C.; Sondheimer, F. Tetrahedron Lett. 1972, 13, 3425-3428. doi:10.1016/S0040-4039(01)94062-6

251. Garratt, P. J.; Nicolaou, K. C.; Sondheimer, F. J. Chem. Soc. D 1970, 1219-1220. doi:10.1039/C29700001219

252.Garratt, P. J.; Nicolaou, K. C.; Sondheimer, F. J. Am. Chem. Soc. 1973, 95, 4582-4592. doi:10.1021/ja00795a021

253. Garratt, P. J.; Nicolaou, K. C.; Sondheimer, F. J. Chem. Soc. D 1971, 1018b-1019. doi:10.1039/c2971001018b

254.Garratt, P. J.; Nicolaou, K. C.; Sondheimer, F. J. Org. Chem. 1973, 38, 2715-2717. doi:10.1021/jo00955a031

255.Jasiobedzki, W.; Kubik, E.; Smolka, G. Rocz. Chem. 1974, 48, 167. Chem. Abstr. 1974, 83,63747s. See for the preparation of another cyclic biscumulene, 1,4,7,10-tetraphenylcyclododeca-1,2,3,7,8,9-hexaene by dimerization of 2,5-diphenylhexa-1,5-dien-3-yne.

256. Ghosh, P.; Zang, Y.; Emge, T. J.; Williams, L. J. Org. Lett. 2009, 11, 4402-4405. doi:10.1021/ol901755a

257. King, R. B.; Harmon, C. A. J. Organomet. Chem. 1975, 86, 239-242. doi:10.1016/S0022-328X(00)89616-3

258. Nagendrappa, G.; Joshi, G. C.; Devaprabhakara, D. J. Organomet. Chem. 1971, 27, 421-426. doi:10.1016/S0022-328X(00)82175-0

259. Vollhardt, K. P. C.; Bergman, R. G. J. Am. Chem. Soc. 1972, 94 , 8950-8951. doi:10.1021/ja00780a075

260. Henry, T. J.; Bergman, R. G. J. Am. Chem. Soc. 1972, 94, 5103-5105. doi:10.1021/ja00769a064

261.Bergman, R. G. Acc. Chem. Res. 1973, 6, 25-31. doi:10.1021/ar50061a004

262. Eisenhuth, L.; Hopf, H. J. Am. Chem. Soc. 1974, 96, 5667-5668. doi:10.1021/ja00824a107

263. Eisenhuth, L.; Hopf, H. Chem. Ber. 1975, 108, 2635-2648. doi:10.1002/cber.19751080819

264. Mitchell, G. H.; Sondheimer, F. J. Am. Chem. Soc. 1969, 91, 7520-7521. doi:10.1021/ja01054a055

265.Wang, K. K.; Liu, B.; Petersen, J. L. J. Am. Chem. Soc. 1996, 118, 6860-6867. doi:10.1021/ja960506a

266. Dower, W. V.; Vollhardt, K. P. C. Tetrahedron 1986, 42, 1873-1881. doi:10.1016/S0040-4020(01)87607-9

267. Dower, W. V.; Vollhardt, K. P. C. Angew. Chem. 1982, 94, 712. doi:10.1002/ange.19820940915 Angew. Chem. Suppl. 1982, 1545-1555.

268. Tanaka, K.; Tomomori, A.; Scott, J. L. Eur. J. Org. Chem. 2003, 2035-2038. doi:10.1002/ejoc.200300092

269. Bui, B. H.; Schreiner, P. R. Eur. J. Org. Chem. 2006, 4187-4192. doi:10.1002/ejoc.200600270

270.Cheng, Y. S. P.; Garratt, P. J.; Neoh, S. B.; Rumjanek, V. M. Isr. J. Chem. 1985, 26, 101-107.

271.Cheng, Y. S. P.; Dominguez, E.; Garratt, P. J.; Neoh, S. B. Tetrahedron Lett. 1978, 19, 691-694. doi:10.1016/S0040-4039(01)85368-5

272.Greenberg, M. M.; Blackstock, S. C.; Berson, J. A.; Merrill, R. A.; Duchamp, J. C.; Zilm, K. W. J. Am. Chem. Soc. 1991, 113, 2318-2319. doi:10.1021/ja00006a069

273.Greenberg, M. M.; Blackstock, S. C.; Berson, J. A. Tetrahedron Lett. 1987, 28, 4263-4266. doi:10.1016/S0040-4039(00)96480-3

274.Braverman, S.; Segev, D. J. Am. Chem. Soc. 1974, 96, 1245-1247. doi:10.1021/ja00811a060 
275.Braverman, S.; Reisman, D. Tetrahedron Lett. 1977, 18, 1753-1756. doi:10.1016/S0040-4039(01)93267-8

And references cited therein.

276. Braverman, S.; Duar, Y.; Segev, D. Tetrahedron Lett. 1976, 17, 3181-3184. doi:10.1016/S0040-4039(00)93874-7

And references cited therein.

277.Braverman, S.; Duar, Y. J. Am. Chem. Soc. 1990, 112, 5830-5837. doi:10.1021/ja00171a024

278. Braverman, S.; Duar, Y. Tetrahedron Lett. 1978, 19, 1493-1496. doi:10.1016/S0040-4039(01)94584-8

279.Braverman, S.; Reisman, D. J. Am. Chem. Soc. 1977, 99, 605-607. doi:10.1021/ja00444a048

280.Braverman, S.; Reisman, D.; Sprecher, M.; Rabinovich, D.; Frolow, F. Tetrahedron Lett. 1979, 20, 901-902. doi:10.1016/S0040-4039(01)93583-X

281. Iwai, I.; Ide, J. Chem. Pharm. Bull. 1964, 12, 1094-1100. doi:10.1248/cpb.12.1094

282.Garratt, P. J.; Neoh, S. B. J. Am. Chem. Soc. 1975, 97, 3255-3257. doi:10.1021/ja00844a075

283.Bartlett, J.; Laird, T.; Ollis, W. D. J. Chem. Soc., Chem. Commun. 1974, 496-497. doi:10.1039/c39740000496

284. Laird, T.; Ollis, W. D. J. Chem. Soc., Chem. Commun. 1972, 557-559. doi:10.1039/c39720000557

285.Montijn, P. P.; Kupecz, A.; Brandsma, L.; Arens, J. F. Recl. Trav. Chim. Pays-Bas 1969, 88, 958-960. doi:10.1002/recl.19690880809

286. Braverman, S.; Zafrani, Y.; Gottlieb, H. E. Tetrahedron 2001, 57, 9177-9185. doi:10.1016/S0040-4020(01)00923-1

287. Braverman, S.; Zafrani, Y.; Gottlieb, H. E. Tetrahedron Lett. 2000, 41, 2675-2678. doi:10.1016/S0040-4039(00)00221-5

288. Braverman, S.; Zafrani, Y.; Gottlieb, H. E. J. Org. Chem. 2002, 67, 3277-3283. doi:10.1021/jo010947z

289.Zafrani, Y.; Gottlieb, H. E.; Sprecher, M.; Braverman, S. J. Org. Chem. 2005, 70, 10166-10168. doi:10.1021/jo051692i

290. Eglinton, G.; Lardy, I. A.; Raphael, R. A.; Sim, G. A. J. Chem. Soc. 1964, 1154-1158. doi:10.1039/jr9640001154

291.Braverman, S.; Cherkinsky, M.; Birsa, M. L.; Zafrani, Y. Eur. J. Org. Chem. 2002, 3198-3207. doi:10.1002/1099-0690(200209)2002:18<3198::AID-EJOC3198>3.0.C O;2-C

292.Zafrani, Y.; Cherkinsky, M.; Gottlieb, H. E.; Braverman, S. Tetrahedron 2003, 59, 2641-2649. doi:10.1016/S0040-4020(03)00327-2

293. Braverman, S.; Freund, M. Tetrahedron 1990, 46, 5759-5776. doi:10.1016/S0040-4020(01)87773-5

294.Birsa, M. L.; Cherkinsky, M.; Braverman, S. Tetrahedron Lett. 2002, 43, 9615-9619. doi:10.1016/S0040-4039(02)02409-7

295.Braverman, S.; Cherkinsky, M.; Goldberg, I.; Sprecher, M. Tetrahedron Lett. 2007, 48, 6713-6716. doi:10.1016/j.tetlet.2007.07.143

296.Braverman, S.; Cherkinsky, M.; Meridor, D.; Sprecher, M. Tetrahedron 2010, 66, 1925-1930. doi:10.1016/j.tet.2010.01.012

297. Braverman, S.; Pechenick-Azizi, T.; Gottlieb, H. E.; Sprecher, M. Synthesis 2011, 1741-1750. doi:10.1055/s-0030-1260024

298. Damour, D.; Pornet, J.; Randrianoelina, B.; Miginiac, L. J. Organomet. Chem. 1990, 396, 289-297. doi:10.1016/0022-328X(90)85470-J

299. Méva'a, L. M.; Pornet, J. Synth. Commun. 1996, 26, 3351-3358. doi:10.1080/00397919608003738
300. In principle the reactions summarized in Scheme 133 could also be initiated photochemically. However, no such photocycloadditions of 605 have been reported in the chemical literature, supporting the observation that the photochemistry of allenes is still an underdeveloped area.

301.Deng, Y.; Fu, C.; Ma, S. Chem.-Eur. J. 2011, 17, 4976-4980. doi:10.1002/chem.201003546

302. Doupeux, H.; Martinet, P.; Simonet, J. Bull. Soc. Chim. Fr. 1971, 2299-2305.

303.Doupeux, H.; Martinet, P.; Simonet, J.; Bretelle, D. Bull. Soc. Chim. Fr. 1970, 3930-3935.

304.Becker, D.; Haddad, N.; Sahali, Y. Tetrahedron Lett. 1989, 30 , 4429-4432. doi:10.1016/S0040-4039(00)99380-8

305.Zimmerman, H. E.; Baker, M. R.; Bottner, R. C.; Morrissey, M. M.; Murphy, S. J. Am. Chem. Soc. 1993, 115, 459-466. doi:10.1021/ja00055a014

306. Kirmse, W.; Strehlke, I. K.; Steenken, S. J. Am. Chem. Soc. 1995, 117, 7007-7008. doi:10.1021/ja00131a026

307.Shepard, M. S.; Carreira, E. M. Tetrahedron 1997, 53, 16253-16276. doi:10.1016/S0040-4020(97)01013-2

308. Krause, N.; Hoffmann-Röder, A. Chapter 18. Allenic Natural Products and Pharmaceuticals. In Modern Allene Chemistry; Krause, N.; Hashmi, A. S. K., Eds.; Wiley, 2008; pp 997-1040. doi:10.1002/9783527619573.ch18

309. Hopf, H. Classics in Hydrocarbon Chemisty: Syntheses, Concepts, Perspectives; Wiley-VCH: Weinheim, Germany, 2000.

\section{License and Terms}

This is an Open Access article under the terms of the Creative Commons Attribution License (http://creativecommons.org/licenses/by/2.0), which permits unrestricted use, distribution, and reproduction in any medium, provided the original work is properly cited.

The license is subject to the Beilstein Journal of Organic Chemistry terms and conditions:

(http://www.beilstein-journals.org/bjoc)

The definitive version of this article is the electronic one which can be found at: doi:10.3762/bjoc. 8.225 